\title{
Definition of the Flexible Image Transport System (FITS), version 3.0
}

\author{
W. D. Pence ${ }^{1}$, L. Chiappetti ${ }^{2}$, C. G. Page ${ }^{3}$, R. A. Shaw ${ }^{4}$, and E. Stobie ${ }^{4}$ \\ ${ }^{1}$ NASA Goddard Space Flight Center, Code 662, Greenbelt, MD 20771, USA \\ e-mail: william.pence@nasa.gov \\ 2 INAF - IASF Milano, via Bassini 15, 20133 Milan, Italy \\ 3 Dept. of Physics \& Astronomy, Univ. of Leicester, University Road, Leicester LE1 7RH, UK \\ ${ }^{4}$ National Optical Astronomy Observatory, 950 N. Cherry Avenue, Tucson, AZ 85719, USA
}

Received 8 July 2010 / Accepted 11 August 2010

\section{ABSTRACT}

\begin{abstract}
The Flexible Image Transport System (FITS) has been used by astronomers for over 30 years as a data interchange and archiving format; FITS files are now handled by a wide range of astronomical software packages. Since the FITS format definition document (the "standard") was last printed in this journal in 2001, several new features have been developed and standardized, notably support for 64-bit integers in images and tables, variable-length arrays in tables, and new world coordinate system conventions which provide a mapping from an element in a data array to a physical coordinate on the sky or within a spectrum. The FITS Working Group of the International Astronomical Union has therefore produced this new version 3.0 of the FITS standard, which is provided here in its entirety. In addition to describing the new features in FITS, numerous editorial changes were made to the previous version to clarify and reorganize many of the sections. Also included are some appendices which are not formally part of the standard. The FITS standard is likely to undergo further evolution, in which case the latest version may be found on the FITS Support Office Web site at http://fits.gsfc.nasa.gov/, which also provides many links to FITS-related resources.
\end{abstract}

Key words. instrumentation: miscellaneous - methods: miscellaneous - techniques: miscellaneous - reference systems standards - astronomical databases: miscellaneous

\section{Foreword}

Since its inception in the late 1970s, the Flexible Image Transport System (FITS) data format has enjoyed wide acceptance in the astronomical community. Indeed, FITS is used as the archival format for most digital archives of astronomical data, FITS is one of the most common run-time file formats for analysis applications, and FITS is seeing continued utility as a component of data formats for the Virtual Observatory. After the initial FITS definition papers appeared (Wells et al. 1981; Greisen \& Harten 1981) the International Astronomical Union (IAU) formally endorsed the FITS format in 1982 and formed the FITS Working Group (FWG) in 1988 to review, approve, and maintain future extensions to FITS. In recognition of the need for a single authoritative source on the correct usage of FITS, a formal standard document was prepared with the support of a technical panel of the NASA/Science Office of Standards and Technology (NOST). In 2000, this FITS standard (version 2.0) was approved by the IAUFWG and was published nearly verbatim in Hanisch et al. (2001).

The FITS standard has evolved in many significant ways during the last decade. In 2005 the IAUFWG approved use of the 64-bit integer data type in FITS images and tables and added support for variable-length arrays within tables. But some of the most significant extensions to FITS did not appear in the standard document per se, but in a series of papers (Greisen \& Calabretta 2002; Calabretta \& Greisen 2002; Greisen et al. 2006) that described formalisms for the support of world coordinate systems (WCSs), i.e., the mapping from a position in a data array to a physical coordinate system such as a position on the sky or within a spectrum. Although the IAUFWG officially recognized the content of these papers as a part of the standard, they were not formally mentioned in the FITS standard document itself.

In late 2006 the IAUFWG appointed a technical panel to address specific ambiguities that had been identified in the standard over the years, to clarify the meaning of some portions of the text, and to consider how to incorporate all the approved elements of the standard into one document. This work was completed by mid-2007, and after an extensive public comment and review process, this version 3.0 of the FITS standard was approved by the IAU FWG in 2008, replacing all prior versions. Yet in spite of the substantial enhancements to FITS that have occurred over the years, maintaining backward and forward compatibility has been a vital guiding principle: "once FITS, always FITS" means that no change to the standard will invalidate a FITS file that was valid at the time it was created. This attribute is essential for long-term curation of digital data archives.

What follows is the verbatim text of the FITS version 3.0 standard, apart from re-numbering the tables in deference to journal style. The text also includes the Appendices, which are not formally a part of the standard, but excludes the table of contents and the index. The first two sections review the history of FITS and define the basic terms used throughout the rest of the document. The next three sections present the basics of FITS, beginning with the overall organization of a FITS file (Sect. 3), which consists of a sequence of header units (Sect. 4) and optional data units (Sect. 5). This is followed by a detailed definition of the Random Groups structure (Sect. 6, now restricted to use with radio interferometry data), and the three standard FITS extension types: image extensions (Sect. 7.1), ASCII table extensions (Sect. 7.2), and binary table extensions (Sect. 7.3). Finally, Sect. 8 provides basic information about the FITS World Coordinate System conventions. 
We caution that this presentation of the standard is for the convenience of the community, and that it may be superseded by newer versions. We refer the reader to the extensive set of resources on the FITS Support Office at NASA/GSFC web site ${ }^{1}$ which contains the latest version of the standard document as well as a comprehensive collection of FITS-related documents, software for validating and viewing FITS files, and sample FITS files from a variety of sources. This web site also provides a registry of common FITS conventions that are in use within the community. Although these conventions are not part of the official standard, they may be helpful to designers of new FITS data products.

\section{Introduction}

\begin{abstract}
An archival format must be utterly portable and selfdescribing, on the assumption that, apart from the transcription device, neither the software nor the hardware that wrote the data will be available when the data are read. "Preserving Scientific Data on our Physical Universe", p. 60. Steering Committee for the Study on the Long-Term Retention of Selected Scientific and Technical Records of the Federal Government, [US] National Research Council, National Academy Press 1995.
\end{abstract}

This document, hereafter referred to as the "standard", describes the Flexible Image Transport System (FITS) which is the standard archival data format for astronomical data sets. Although FITS was originally designed for transporting image data on magnetic tape (which accounts for the "I" and "T" in the name), the capabilities of the FITS format have expanded to accommodate more complex data structures. The role of FITS has also grown from simply a way to transport data between different analysis software systems into the preferred format for data in astronomical archives, as well as the on-line analysis format used by many software packages.

This standard is intended as a formal codification of the FITS format which has been endorsed by the International Astronomical Union (IAU) for the interchange of astronomical data (IAU 1983). It is fully consistent with all actions and endorsements of the IAU FITS Working Group (IAUFWG) which was appointed by Commission 5 of the IAU to oversee further development of the FITS format. In particular, this standard defines the organization and content of the header and data units for all standard FITS data structures: the primary array, the random groups structure, the image extension, the ASCII table extension, and the binary table extension. It also specifies minimum structural requirements and general principles governing the creation of new extensions. For headers, it specifies the proper syntax for keyword records and defines required and reserved keywords. For data, it specifies character and numeric value representations and the ordering of contents within the byte stream.

One important feature of the FITS format is that its structure, down to the bit level, is completely specified in documents (such as this standard), many of which have been published in refereed scientific journals. Given these documents, which are readily available in hard copy form in libraries around the world as well as in electronic form on the Internet, future researchers should be able to decode the stream of bytes in any FITS format data file. In contrast, many other current data formats are only implicitly defined by the software that read and write the files.

\footnotetext{
1 http://fits.gsfc.nasa.gov/
}

If that software is not continually maintained so that it can be run on future computer systems, then the information encoded in those data files could be lost.

\subsection{Brief history of FITS}

The FITS format evolved out of the recognition that a standard format was needed for transferring astronomical images from one research institution to another. The first prototype developments of a universal interchange format that would eventually lead to the definition of the FITS format began in 1976 between Don Wells at KPNO and Ron Harten at the Netherlands Foundation for Research in Astronomy (NFRA). This need for an image interchange format was raised at a meeting of the Astronomy section of the US National Science Foundation in January 1979, which led to the formation of a task force to work on the problem. Most of the technical details of the first basic FITS agreement (with files consisting of only a primary header followed by a data array) were subsequently developed by Don Wells and Eric Greisen (NRAO) in March 1979. After further refinements, and successful image interchange tests between observatories that used widely different types of computer systems, the first papers that defined the FITS format were published in 1981 (Wells et al. 1981; Greisen \& Harten 1981). The FITS format quickly became the defacto standard for data interchange within the astronomical community (mostly on 9-track magnetic tape at that time) and was officially endorsed by the IAU in 1982 (IAU 1983). Most national and international astronomical projects and organizations subsequently adopted the FITS format for distribution and archiving of their scientific data products. Some of the highlights in the developmental history of FITS are shown in Table 1.

\subsection{Version history of this document}

The fundamental definition of the FITS format was originally contained in a series of published papers (Wells et al. 1981; Greisen \& Harten 1981; Grosbøl et al. 1988; Harten et al. 1988). As FITS became more widely used, the need for a single document to unambiguously define the requirements of the FITS format became apparent. In 1990, the NASA Science Office of Standards and Technology (NOST) at the Goddard Space Flight Center provided funding for a technical panel to develop the first version of this standard document. As shown in Table 2, the NOST panel produced several draft versions, culminating in the first NOST standard document, NOST 100-1.0, in 1993. Although this document was developed under a NASA accreditation process, it was subsequently formally approved by the IAUFWG, which is the international control authority for the FITS format. The small update to the standard in 1995 (NOST 100-1.1) added a recommendation on the physical units of header keyword values.

The NOST technical panel was convened a second time to make further updates and clarifications to the standard, resulting in the NOST 100-2.0 version that was approved by the IAUFWG in 1999 and published in 2001 (Hanisch et al. 2001). In 2005, the IAUFWG formally approved the variable-length array convention in binary tables, and a short time later approved support for the 64-bit integers data type. New versions of the standard were released to reflect both of these changes (versions IAUFWG 2.1 and IAUFWG 2.1b).

Most recently, the IAUFWG appointed its own technical panel in early 2007 to consider further modifications and updates to the standard. The changes proposed by this panel, which 
Table 1. Significant milestones in the development of FITS.

\begin{tabular}{lll}
\hline \hline Date & Milestone & Section \\
\hline 1979 & Initial FITS Agreement and first interchange of files & \\
1981 & Published original (single HDU) definition (Wells et al. 1981) & Sect. 6 \\
1981 & Published random groups definition (Greisen \& Harten 1981) & \\
1982 & Formally endorsed by the IAU (IAU 1983) & \\
1988 & Defined rules for multiple extensions (Grosbøl et al. 1988) & Sect. 7.2 \\
1988 & IAU FITS Working Group (IAUFWG) established & Sect. 7.2 \\
1988 & Extended to include ASCII table extensions (Harten et al. 1988) & Sect. 5.3 \\
1988 & Formal IAU approval of ASCII tables (IAU 1988) & Sect. 7.1 \\
1990 & Extended to include IEEE floating-point data (Wells \& Grosbøl 1990) & Sect. 7.3 \\
1994 & Extended to multiple IMAGE array extensions (Ponz et al. 1994) & Sect. 4.4.2 \\
1995 & Extended to binary table extensions (Cotton et al. 1995) & Sect. 8 \\
1997 & Adopted 4-digit year date format (Bunclark \& Rots 1997) & Sect. 8.3 \\
2002 & Adopted conventions for world coordinate systems (Greisen \& Calabretta 2002) \\
2002 & Adopted conventions for celestial coordinates (Calabretta \& Greisen 2002) & App. G \\
2004 & Adopted MIME types for FITS data files (Allen \& Wells 2005) & Sect. 7.3.5 \\
2005 & Extended to support variable-length arrays in binary tables & Sect. 8.4 \\
2005 & Adopted conventions for spectral coordinate systems (Greisen et al. 2006) & Sect. 5.2.4 \\
2005 & Extended to include 64-bit integer data type & \\
\hline
\end{tabular}

Table 2. Version history of the standard.

\begin{tabular}{lll}
\hline \hline Version & Date & Status \\
\hline NOST 100-0.1 & 1990 December & First Draft Standard \\
NOST 100-0.2 & 1991 June & Second Revised Draft Standard \\
NOST 100-0.3 & 1991 December & Third Revised Draft Standard \\
NOST 100-1.0 & 1993 June & NOST Standard \\
NOST 100-1.1 & 1995 September & NOST Standard \\
NOST 100-2.0 & 1999 March & NOST Standard \\
IAUFWG 2.1 & 2005 April & IAUFWG Standard \\
IAUFWG 2.1b & 2005 December & IAUFWG Standard \\
IAUFWG 3.0 & 2008 July & IAUFWG Standard \\
\hline
\end{tabular}

were ultimately approved by the IAUFWG after a formal public review process, are shown in this 3.0 version of the document.

The latest version of the standard, as well as other information about the FITS format, can be obtained from the FITS Support Office web site at http://fits.gsfc.nasa. gov. This web site also contains the contact information for the Chairman of the IAUFWG, to whom any questions or comments regarding this standard should be addressed.

\subsection{Acknowledgments}

The members of the three technical panels that produced this standard are shown below.

First technical panel, 1990-1993

Robert J. Hanisch (Chair) Space Telescope Science Inst.

Lee E. Brotzman Hughes STX

Edward Kemper

Hughes STX

Barry M. Schlesinger

Peter J. Teuben

Raytheon STX

University of Maryland

Michael E. Van Steenberg NASA Goddard SFC

Wayne H. Warren Jr. Hughes STX

Richard A. White NASA Goddard SFC

\section{Second technical panel, 1994-1999}

Robert J. Hanisch (Chair) Space Telescope Science Inst.
Allen Farris

Eric W. Greisen

William D. Pence

Barry M. Schlesinger

Peter J. Teuben
Randall W. Thompson

Archibald Warnock

Computer Sciences Corp. A/WWW Enterprises

Third technical panel, 2007

William D. Pence (Chair) NASA Goddard SFC

Lucio Chiappetti IASF Milano, INAF, Italy

Clive G. Page

Richard Shaw

Elizabeth Stobie University of Arizona National Optical Astr. Obs.

\section{Definitions, acronyms, and symbols}

\subsection{Conventions used in this document}

Terms or letters set in Courier font represent literal strings that appear in FITS files. In the case of keyword names, such as "NAXISn", the lower case letter represents a positive integer index number, generally in the range 1 to 999 . The emphasized words must, shall, should, may, recommended, and optional in this document are to be interpreted as described in IETF standard, RFC 2119 (Bradner 1997).

\subsection{Defined terms}

ـ Used to designate an ASCII space character.

ANSI. American National Standards Institute.

Array. A sequence of data values. This sequence corresponds to the elements in a rectilinear, $n$-dimension matrix $(1 \leq n \leq$ 999 , or $n=0$ in the case of a null array). 
Array value. The value of an element of an array in a FITS file, without the application of the associated linear transformation to derive the physical value.

ASCII. American National Standard Code for Information Interchange.

ASCII character. Any member of the 7-bit ASCII character set.

ASCII digit. One of the ten ASCII characters " 0 " through " 9 " which are represented by decimal character codes 48 through 57 (hexadecimal 30 through 39).

ASCII NULL. The ASCII character that has all eight bits set to zero.

ASCII space. The ASCII character for space which is represented by decimal 32 (hexadecimal 20).

ASCII text. The restricted set of ASCII characters decimal 32 through 126 (hexadecimal 20 through 7E).

Basic FITS. The FITS structure consisting of the primary header followed by a single primary data array. This is also known as Single Image FITS (SIF), as opposed to MultiExtension FITS (MEF) files that contain one or more extensions following the primary HDU.

Big endian. The numerical data format used in FITS files in which the most significant byte of the value is stored first followed by the remaining bytes in order of significance.

Bit. A single binary digit.

Byte. An ordered sequence of eight consecutive bits treated as a single entity.

Card image. An obsolete term for an 80-character keyword record derived from the 80 -column punched computer cards that were prevalent in the 1960s and 1970s.

Character string. A sequence of one or more of the restricted set of ASCII text characters, decimal 32 through 126 (hexadecimal 20 through $7 \mathrm{E}$ ).

Conforming extension. An extension whose keywords and organization adhere to the requirements for conforming extensions defined in Sect. 3.4.1 of this standard.

Data block. A 2880-byte FITS block containing data described by the keywords in the associated header of that HDU.

Deprecate. To express disapproval of. This term is used to refer to obsolete structures that should not be used in new FITS files but which shall remain valid indefinitely.

Entry. A single value in an ASCII table or binary table standard extension.

Extension. A FITS HDU appearing after the primary HDU in a FITS file.

Extension type name. The value of the XTENSION keyword, used to identify the type of the extension.

Field. A component of a larger entity, such as a keyword record or a row of an ASCII table or binary table standard extension. A field in a table extension row consists of a set of zero or more table entries collectively described by a single format.

File. A sequence of one or more records terminated by an endof-file indicator appropriate to the medium.

FITS. Flexible Image Transport System.

FITS block. A sequence of 2880 8-bit bytes aligned on 2880 byte boundaries in the FITS file, most commonly either a header block or a data block. Special records are another infrequently used type of FITS block. This block length was chosen because it is evenly divisible by the byte and word lengths of all known computer systems at the time FITS was developed in 1979.

FITS file. A file with a format that conforms to the specifications in this document.
FITS structure. One of the components of a FITS file: the primary HDU, the random groups records, an extension, or, collectively, the special records following the last extension.

FITS Support Office. The FITS information web site that is maintained by the IAUFWG and is currently hosted at http://fits.gsfc.nasa.gov.

Floating point. A computer representation of a real number.

Fraction. The field of the mantissa (or significand) of a floatingpoint number that lies to the right of its implied binary point.

Group parameter value. The value of one of the parameters preceding a group in the random groups structure, without the application of the associated linear transformation.

HDU. Header and Data Unit. A data structure consisting of a header and the data the header describes. Note that an HDU may consist entirely of a header with no data blocks.

Header. A series of keyword records organized within one or more header blocks that describes structures and/or data which follow it in the FITS file.

Header block. A 2880-byte FITS block containing a sequence of thirty-six 80-character keyword records.

Heap. The supplemental data area following the main data table in a binary table standard extension.

IAU. International Astronomical Union.

IAUFWG. International Astronomical Union FITS Working Group.

IEEE. Institute of Electrical and Electronic Engineers.

IEEE NaN. IEEE Not-a-Number value; used to represent undefined floating-point values in FITS arrays and binary tables.

IEEE special values. Floating-point number byte patterns that have a special, reserved meaning, such as $-0, \pm \infty$, \pm underflow, \pm overflow, \pm denormalized, \pm NaN. (See Appendix E).

Indexed keyword. A keyword name that is of the form of a fixed root with an appended positive integer index number.

Keyword name. The first eight bytes of a keyword record which contain the ASCII name of a metadata quantity (unless it is blank).

Keyword record. An 80-character record in a header block consisting of a keyword name in the first eight characters followed by an optional value indicator, value and comment string. The keyword record shall be composed only of the restricted set of ASCII text characters ranging from decimal 32 to 126 (hexadecimal 20 to $7 \mathrm{E}$ ).

Mandatory keyword. A keyword that must be used in all FITS files or a keyword required in conjunction with particular FITS structures.

Mantissa. Also known as significand. The component of an IEEE floating-point number consisting of an explicit or implicit leading bit to the left of its implied binary point and a fraction field to the right.

MEF. Multi-Extension FITS, i.e., a FITS file containing a primary HDU followed by one or more extension HDUs.

NOST. NASA/Science Office of Standards and Technology.

Physical value. The value in physical units represented by an element of an array and possibly derived from the array value using the associated, but optional, linear transformation.

Pixel. Short for "Picture element"; a single location within an array.

Primary data array. The data array contained in the primary HDU.

Primary HDU. The first HDU in a FITS file. 
Primary header. The first header in a FITS file, containing information on the overall contents of the file (as well as on the primary data array, if present).

Random Group. A FITS structure consisting of a collection of "groups", where a group consists of a subarray of data and a set of associated parameter values. Random groups are deprecated for any use other than for radio interferometry data.

Record. A sequence of bits treated as a single logical entity.

Repeat count. The number of values represented in a field in a binary table standard extension.

Reserved keyword. An optional keyword that must be used only in the manner defined in this standard.

SIF. Single Image FITS, i.e., a FITS file containing only a primary HDU, without any extension HDUs. Also known as Basic FITS.

Special records. A series of one or more FITS blocks following the last HDU whose internal structure does not otherwise conform to that for the primary HDU or to that specified for a conforming extension in this standard. Any use of special records requires approval from the IAU FITS Working Group.

Standard extension. A conforming extension whose header and data content are completely specified in Sect. 7 of this standard, namely, an image extension, an ASCII table extension, or a binary table extension.

\section{FITS file organization}

\subsection{Overall file structure}

A FITS file shall be composed of the following FITS structures, in the order listed:

- Primary header and data unit (HDU).

- Conforming Extensions (optional).

- Other special records (optional, restricted).

A FITS file composed of only the primary HDU is sometimes referred to as a Basic FITS file, or a Single Image FITS (SIF) file, and a FITS file containing one or more extensions following the primary HDU is sometimes referred to as a Multi-Extension FITS (MEF) file.

Each FITS structure shall consist of an integral number of FITS blocks which are each 2880 bytes (23040 bits) in length. The primary HDU shall start with the first FITS block of the FITS file. The first FITS block of each subsequent FITS structure shall be the FITS block immediately following the last FITS block of the preceding FITS structure.

This standard does not impose a limit on the total size of a FITS file, nor on the size of an individual HDU within a FITS file. Software packages that read or write data according to this standard could be limited, however, in the size of files that are supported. In particular, some software systems have historically only supported files up to $2^{31}$ bytes in size (approximately $2.1 \times 10^{9}$ bytes).

\subsection{Individual FITS structures}

The primary HDU and every extension HDU shall consist of one or more 2880-byte header blocks immediately followed by an optional sequence of associated 2880-byte data blocks. The header blocks shall contain only the restricted set of ASCII text characters, decimal 32 through 126 (hexadecimal 20 through 7E). The ASCII control characters with decimal values less than 32 (including the null, tab, carriage return, and line feed characters), and the delete character (decimal 127 or hexadecimal 7F) must not appear anywhere within a header block.

\subsection{Primary header and data unit}

The first component of a FITS file shall be the primary HDU which always contains the primary header and may be followed by the primary data array. If the primary data array has zero length, as determined by the values of the NAXIS and NAXISn keywords in the primary header (Sect. 4.4.1), then the primary HDU shall contain no data blocks.

\subsubsection{Primary header}

The header of a primary HDU shall consist of one or more header blocks, each containing a series of 80-character keyword records containing only the restricted set of ASCII text characters. Each 2880-byte header block contains 36 keyword records. The last header block must contain the END keyword (defined in Sect. 4.4.1) which marks the logical end of the header. Keyword records without information (e.g., following the END keyword) shall be filled with ASCII spaces (decimal 32 or hexadecimal 20).

\subsubsection{Primary data array}

The primary data array, if present, shall consist of a single data array with from 1 to 999 dimensions (as specified by the NAXIS keyword defined in Sect. 4.4.1). The random groups convention in the primary data array is a more complicated structure and is discussed separately in Sect. 6. The entire array of data values are represented by a continuous stream of bits starting with the first bit of the first data block. Each data value shall consist of a fixed number of bits that is determined by the value of the BITPIX keyword (Sect. 4.4.1). Arrays of more than one dimension shall consist of a sequence such that the index along axis 1 varies most rapidly, that along axis 2 next most rapidly, and those along subsequent axes progressively less rapidly, with that along axis $m$, where $m$ is the value of NAXIS, varying least rapidly. There is no space or any other special character between the last value on a row or plane and the first value on the next row or plane of a multi-dimensional array. Except for the location of the first element, the array structure is independent of the FITS block structure. This storage order is shown schematically in Fig. 1 and is the same order as in multi-dimensional arrays in the Fortran programming language (ISO 2004). The index count along each axis shall begin with 1 and increment by 1 up to the value of the NAXISn keyword (Sect. 4.4.1).

If the data array does not fill the final data block, the remainder of the data block shall be filled by setting all bits to zero. The individual data values shall be stored in big-endian byte order such that the byte containing the most significant bits of the value appears first in the FITS file, followed by the remaining bytes, if any, in decreasing order of significance.

\subsection{Extensions}

\subsubsection{Requirements for conforming extensions}

All extensions, whether or not further described in this standard, shall fulfill the following requirements to be in conformance with this FITS standard. New extension types should be created only when the organization of the information is such that it cannot be handled by one of the existing extension types. A FITS 


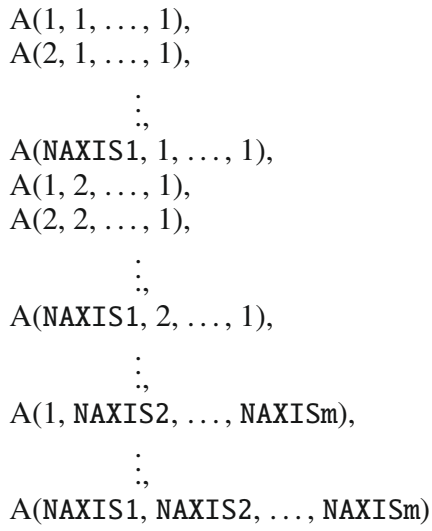

Fig. 1. Arrays of more than one dimension shall consist of a sequence such that the index along axis 1 varies most rapidly and those along subsequent axes progressively less rapidly.

file that contains extensions is commonly referred to as a multiextension FITS (MEF) file.

3.4.1.1. Identity. Each extension type shall have a unique type name, specified in the header by the XTENSION keyword (Sect. 4.4.1). To preclude conflict, extension type names must be registered with the IAUFWG. The current list of registered extensions is given in Appendix F. An up-to-date list is also maintained on the FITS Support Office web site.

3.4.1.2. Size specification. The total number of bits in the data of each extension shall be specified in the header for that extension, in the manner prescribed in Sect. 4.4.1.

\subsubsection{Standard extensions}

A standard extension is a conforming extension whose organization and content are completely specified in Sect. 7 of this standard. Only one extension format shall be approved for each type of data organization.

\subsubsection{Order of extensions}

An extension may follow the primary HDU or another conforming extension. Standard extensions and other conforming extensions may appear in any order in a FITS file.

\subsection{Special records (restricted use)}

Special records are 2880-byte FITS blocks following the last HDU of the FITS file that have an unspecified structure that does not meet the requirements of a conforming extension. The first 8 bytes of the special records must not contain the string "XTENSION". It is recommended that they do not contain the string "SIMPLE $\sqcup$ ". The contents of special records are not otherwise specified by this standard.

Special records were originally designed as a way for the FITS format to evolve by allowing new FITS structures to be implemented. Following the development of conforming extensions, which provide a general mechanism for storing different types of data structures in FITS format in a well defined manner, the need for other new types of FITS data structures has been greatly reduced. Consequently, further use of special records is restricted and requires the approval of the IAU FITS Working Group.

\subsection{Physical blocking}

\subsubsection{Bitstream devices}

For bitstream devices, including but not restricted to logical file systems, FITS files shall be interpreted as a sequence of one or more 2880-byte FITS blocks, regardless of the physical blocking structure of the underlying recording media. When writing a FITS file on media with a physical block size unequal to the 2880-byte FITS block length, any bytes remaining in the last physical block following the end of the FITS file should be set to zero. Similarly, when reading FITS files on such media, any bytes remaining in the last physical block following the end of the FITS file shall be disregarded.

\subsubsection{Sequential media}

The FITS format was originally developed for writing files on sequential magnetic tape devices. The following rules on how to write to sequential media (Grosbøl \& Wells 1994) are now irrelevant to most current data storage devices.

If physically possible, FITS files shall be written on sequential media in blocks that are from one to ten integer multiples of 2880-bytes in length. If this is not possible, the FITS file shall be written as a bitstream using the native block size of the sequential device. Any bytes remaining in the last block following the end of the FITS file shall be set to zero.

When reading FITS files on sequential media, any files shorter than 2880 bytes in length (e.g., ANSI tape labels) are not considered part of the FITS files and should be disregarded.

\subsection{Restrictions on changes}

Any structure that is a valid FITS structure shall remain a valid FITS structure at all future times. Use of certain valid FITS structures may be deprecated by this or future FITS standard documents.

\section{Headers}

The first two sections of this chapter define the structure and content of header keyword records. This is followed in Sect. 4.3 with recommendations on how physical units should be expressed. The final section defines the mandatory and reserved keywords for primary arrays and conforming extensions.

\subsection{Keyword records}

\subsubsection{Syntax}

Each 80-character header keyword record shall consist of a keyword name, a value indicator (only required if a value is present), an optional value, and an optional comment. Keywords may appear in any order except where specifically stated otherwise in this standard. It is recommended that the order of the keywords in FITS files be preserved during data processing operations because the designers of the FITS file may have used conventions that attach particular significance to the order of certain keywords (e.g., by grouping sequences of COMMENT keywords 
at specific locations in the header, or appending HISTORY keywords in chronological order of the data processing steps).

A formal syntax, giving a complete definition of the syntax of FITS keyword records, is given in Appendix A. It is intended as an aid in interpreting the text defining the standard.

\subsubsection{Components}

4.1.2.1. Keyword name (bytes 1 through 8). The keyword name shall be a left justified, 8-character, space-filled, ASCII string with no embedded spaces. All digits 0 through 9 (decimal ASCII codes 48 to 57, or hexadecimal 30 to 39) and upper case Latin alphabetic characters "A" through " $Z$ " (decimal 65 to 90 or hexadecimal 41 to $5 \mathrm{~A}$ ) are permitted; lower case characters shall not be used. The underscore (“_”, decimal 95 or hexadecimal 5F) and hyphen ("-", decimal 45 or hexadecimal 2D) are also permitted. No other characters are permitted ${ }^{2}$. For indexed keyword names that have a single positive integer index counter appended to the root name, the counter shall not have leading zeroes (e.g., NAXIS1, not NAXISOQ1). Note that keyword names that begin with (or consist solely of) any combination of hyphens, underscores, and digits are legal.

4.1.2.2. Value indicator (bytes 9 and 10). If the two ASCII characters " $=\sqcup "$ " (decimal 61 followed by decimal 32$)$ are present in bytes 9 and 10 of the keyword record this indicates that the keyword has a value field associated with it, unless it is one of the commentary keywords defined in Sect. 4.4 .2 (i.e., a HISTORY, COMMENT, or completely blank keyword name) which by definition have no value.

4.1.2.3. Value/comment (bytes 11 through 80). In keyword records that contain the value indicator in bytes 9 and 10, the remaining bytes 11 through 80 of the record shall contain the value, if any, of the keyword, followed by optional comments. In keyword records without a value indicator, bytes 9 through 80 should be interpreted as commentary text, however, this does not preclude conventions that interpret the content of these bytes in other ways.

The value field, when present, shall contain the ASCII text representation of a literal string constant, a logical constant, or a numerical constant, in the format specified in Sect. 4.2. The value field may be a null field; i.e., it may consist entirely of spaces, in which case the value associated with the keyword is undefined.

The mandatory FITS keywords defined in this standard must not appear more than once within a header. All other keywords that have a value should not appear more than once. If a keyword does appear multiple times with different values, then the value is indeterminate.

If a comment follows the value field, it must be preceded by a slash ("/", decimal 47 or hexadecimal 2F) ${ }^{2}$. A space between the value and the slash is strongly recommended. The comment may contain any of the restricted set of ASCII text characters, decimal 32 through 126 (hexadecimal 41 through 7E). The ASCII control characters with decimal values less than 32 (including the null, tab, carriage return, and line feed characters), and the delete character (decimal 127 or hexadecimal 7F) must not appear anywhere within a keyword record.

\footnotetext{
2 This requirement differs from the wording in the original FITS papers. See Appendix H.
}

\subsection{Value}

The structure of the value field depends on the data type of the value. The value field represents a single value and not an array of values ${ }^{2}$. The value field must be in one of two formats: fixed or free. The fixed-format is required for values of mandatory keywords and is recommended for values of all other keywords.

\subsubsection{Character string}

A character string value shall be composed only of the set of restricted ASCII text characters, decimal 32 through 126 (hexadecimal 20 through 7E) enclosed by single quote characters (“" ", decimal 39, hexadecimal 27). A single quote is represented within a string as two successive single quotes, e.g., O'HARA = ' 0 ' 'HARA'. Leading spaces are significant; trailing spaces are not. This standard imposes no requirements on the case sensitivity of character string values unless explicitly stated in the definition of specific keywords.

If the value is a fixed-format character string, the starting single quote character must be in byte 11 of the keyword record and the closing single quote must occur in or before byte 80 . Earlier versions of this standard also required that fixed-format characters strings must be padded with space characters to at least a length of eight characters so that the closing quote character does not occur before byte 20 . This minimum character string length is no longer required, except for the value of the XTENSION keyword (e.g., 'IMAGE $\sqcup \sqcup \sqcup$ ' and 'TABLE must be padded to a length of eight characters for backward compatibility with previous usage.

Free-format character strings follow the same rules as fixedformat character strings except that the starting single quote character may occur after byte 11 . Any bytes preceding the starting quote character and after byte 10 must contain the space character.

Note that there is a subtle distinction between the following three keywords:

$\begin{array}{ll}\text { KEYWORD1 }= & , \quad, \quad, \quad / \text { null string keyword } \\ \text { KEYWORD2 }= & \text { / empty string keyword } \\ \text { KEYWORD3 }= & \text { / undefined keyword }\end{array}$

The value of KEYWORD1 is a null, or zero length string whereas the value of the KEYWORD2 is an empty string (nominally a single space character because the first space in the string is significant, but trailing spaces are not). The value of KEYWORD3 is undefined and has an indeterminate data type as well, except in cases where the data type of the specified keyword is explicitly defined in this standard.

The maximum possible length of a keyword string is 68 characters (with the opening and closing quote characters in bytes 11 and 80, respectively). In general, no length limit less than 68 is implied for character-valued keywords.

\subsubsection{Logical}

If the value is a fixed-format logical constant, it shall appear as an uppercase $\mathrm{T}$ or $\mathrm{F}$ in byte 30 . A logical value is represented in free-format by a single character consisting of an uppercase $\mathrm{T}$ or $\mathrm{F}$ as the first non-space character in bytes 11 through 80 . 


\subsubsection{Integer number}

If the value is a fixed-format integer, the ASCII representation shall be right-justified in bytes 11 through 30 . An integer consists of a "+" (decimal 43 or hexadecimal 2B) or "-" (decimal 45 or hexadecimal 2D) sign, followed by one or more contiguous ASCII digits (decimal 48 to 57 or hexadecimal 30 to 39), with no embedded spaces. The leading "+" sign is optional. Leading zeros are permitted, but are not significant. The integer representation shall always be interpreted as a signed, decimal number. This standard does not limit the range of an integer keyword value, however, software packages that read or write data according to this standard could be limited in the range of values that are supported (e.g., to the range that can be represented by a 32-bit or 64-bit signed binary integer).

A free-format integer value follows the same rules as fixedformat integers except that the ASCII representation may occur anywhere within bytes 11 through 80 .

\subsubsection{Real floating-point number}

If the value is a fixed-format real floating-point number, the ASCII representation shall be right-justified in bytes 11 through 30 .

A floating-point number is represented by a decimal number followed by an optional exponent, with no embedded spaces. A decimal number shall consist of a "+" (decimal 43 or hexadecimal 2B) or "-" (decimal 45 or hexadecimal 2D) sign, followed by a sequence of ASCII digits containing a single decimal point (“.”), representing an integer part and a fractional part of the floating-point number. The leading "+" sign is optional. At least one of the integer part or fractional part must be present. If the fractional part is present, the decimal point must also be present. If only the integer part is present, the decimal point may be omitted, in which case the floating-point number is indistinguishable from an integer. The exponent, if present, consists of an exponent letter followed by an integer. Letters in the exponential form ("E" or "D") ${ }^{3}$ shall be upper case. The full precision of 64-bit values cannot be expressed over the whole range of values using the fixed-format. This standard does not impose an upper limit on the number of digits of precision, nor any limit on the range of floating-point keyword values. Software packages that read or write data according to this standard could be limited, however, in the range of values and exponents that are supported (e.g., to the range that can be represented by a 32-bit or 64-bit floating-point number).

A free-format floating-point value follows the same rules as a fixed-format floating-point value except that the ASCII representation may occur anywhere within bytes 11 through 80 .

\subsubsection{Complex integer number}

There is no fixed-format for complex integer numbers ${ }^{4}$.

If the value is a complex integer number, the value must be represented as a real part and an imaginary part, separated by a comma and enclosed in parentheses e.g., $(123,45)$. Spaces may precede and follow the real and imaginary parts. The real

\footnotetext{
3 The "D" exponent form is traditionally used when representing values that have more decimals of precision or a larger magnitude than can be represented by a single precision 32-bit floating-point number, but otherwise there is no distinction between " $\mathrm{E}$ " or " $\mathrm{D}$ ".

${ }^{4}$ This requirement differs from the wording in the original FITS papers. See Appendix H.
}

and imaginary parts are represented in the same way as integers (Sect. 4.2.3). Such a representation is regarded as a single value for the complex integer number. This representation may be located anywhere within bytes 11 through 80 .

\subsubsection{Complex floating-point number}

There is no fixed-format for complex floating-point numbers ${ }^{4}$.

If the value is a complex floating-point number, the value must be represented as a real part and an imaginary part, separated by a comma and enclosed in parentheses, e.g., (123.23, -45.7). Spaces may precede and follow the real and imaginary parts. The real and imaginary parts are represented in the same way as floating-point values (Sect. 4.2.4). Such a representation is regarded as a single value for the complex floating-point number. This representation may be located anywhere within bytes 11 through 80 .

\subsection{Units}

When a numerical keyword value represents a physical quantity, it is recommended that units be provided. Units shall be represented with a string of characters composed of the restricted ASCII text character set. Unit strings can be used as values of keywords (e.g., for the reserved keywords BUNIT, and TUNITn), as an entry in a character string column of an ASCII or binary table extension, or as part of a keyword comment string (see Sect. 4.3.2, below).

The units of all FITS header keyword values, with the exception of measurements of angles, should conform with the recommendations in the IAU Style Manual (McNally 1988). For angular measurements given as floating-point values and specified with reserved keywords, the units should be degrees (i.e., deg). If a requirement exists within this standard for the units of a keyword, then those units must be used.

The units for fundamental physical quantities recommended by the IAU are given in Table 3, and additional units that are commonly used in astronomy are given in Table 4 . The recommended plain text form for the IAU-recognized base units are given in Col. 2 of both tables ${ }^{5}$. All base units strings may be preceded, with no intervening spaces, by a single character (two for deca) taken from Table 5 and representing scale factors mostly in steps of $10^{3}$. Compound prefixes (e.g., ZYeV for $10^{45} \mathrm{eV}$ ) must not be used.

\subsubsection{Construction of units strings}

Compound units strings may be formed by combining strings of base units (including prefixes, if any) with the recommended syntax described in Table 6. Two or more base units strings (called str1 and str2 in Table 6) may be combined using the restricted set of (explicit or implicit) operators that provide for multiplication, division, exponentiation, raising arguments to powers, or taking the logarithm or square-root of an argument. Note that functions such as $\log$ actually require dimensionless arguments, so that $\log (\mathrm{Hz})$, for example, actually means $\log (x / 1 \mathrm{~Hz})$. The final units string is the compound string, or a compound of compounds, preceded by an optional numeric multiplier of the form $10^{* * k}, 10^{\wedge} k$, or $10 \pm k$ where $k$ is an integer, optionally surrounded by parentheses with the sign character required in the third form in the absence of

\footnotetext{
5 These tables are reproduced from the first in a series of papers on world coordinate systems (Greisen \& Calabretta 2002), which provides examples and expanded discussion.
} 
Table 3. IAU-recommended basic units.

\begin{tabular}{|c|c|c|c|}
\hline Quantity & Unit & Meaning & Notes \\
\hline \multicolumn{4}{|c|}{ SI base $\mathcal{E}$ supplementary units } \\
\hline length & $\mathrm{m}$ & meter & \\
\hline mass & $\mathrm{kg}$ & kilogram & g gram allowed \\
\hline time & $\mathrm{s}$ & second & \\
\hline plane angle & rad & radian & \\
\hline solid angle & sr & steradian & \\
\hline temperature & $\mathrm{K}$ & kelvin & \\
\hline electric current & A & ampere & \\
\hline amount of substance & mol & mole & \\
\hline luminous intensity & $\mathrm{cd}$ & candela & \\
\hline \multicolumn{4}{|c|}{$I A U$-recognized derived units } \\
\hline frequency & $\mathrm{Hz}$ & hertz & $\mathrm{s}^{-1}$ \\
\hline energy & J & joule & $\mathrm{N} \mathrm{m}$ \\
\hline power & W & watt & $\mathrm{J} \mathrm{s}^{-1}$ \\
\hline electric potential & $\mathrm{V}$ & volt & $\mathrm{J} \mathrm{C}^{-1}$ \\
\hline force & $\mathrm{N}$ & newton & $\mathrm{kg} \mathrm{m} \mathrm{s}{ }^{-2}$ \\
\hline pressure, stress & $\mathrm{Pa}$ & pascal & $\mathrm{N} \mathrm{m}^{-2}$ \\
\hline electric charge & $\mathrm{C}$ & coulomb & A s \\
\hline electric resistance & $\mathrm{Ohm}$ & ohm & $\mathrm{V} \mathrm{A}^{-1}$ \\
\hline electric conductance & $\mathrm{S}$ & siemens & $\mathrm{A} \mathrm{V} \mathrm{V}^{-1}$ \\
\hline electric capacitance & $\mathrm{F}$ & farad & $\mathrm{C} \mathrm{V}^{-1}$ \\
\hline magnetic flux & $\mathrm{Wb}$ & weber & $\mathrm{Vs}$ \\
\hline magnetic flux density & $\mathrm{T}$ & tesla & $\mathrm{Wb} \mathrm{m}^{-2}$ \\
\hline inductance & $\mathrm{H}$ & henry & $\mathrm{Wb} \mathrm{A}^{-1}$ \\
\hline luminous flux & $\operatorname{lm}$ & lumen & $\mathrm{cd} \mathrm{sr}$ \\
\hline illuminance & $1 \mathrm{x}$ & lux & $\operatorname{lm~m}^{-2}$ \\
\hline
\end{tabular}

parentheses. Creators of FITS files are encouraged to use the numeric multiplier only when the available standard scale factors of Table 5 will not suffice. Parentheses are used for symbol grouping and are strongly recommended whenever the order of operations might be subject to misinterpretation. A space character implies multiplication which can also be conveyed explicitly with an asterisk or a period. Therefore, although spaces are allowed as symbol separators, their use is discouraged. Note that, per IAU convention, case is significant throughout. The IAU style manual forbids the use of more than one slash (")") character in a units string. However, since normal mathematical precedence rules apply in this context, more than one slash may be used but is discouraged.

A unit raised to a power is indicated by the unit string followed, with no intervening spaces, by the optional symbols $* *$ or ${ }^{\wedge}$ followed by the power given as a numeric expression, called expr in Table 6. The power may be a simple integer, with or without sign, optionally surrounded by parentheses. It may also be a decimal number (e.g., 1.5, 0.5) or a ratio of two integers (e.g., 7/9), with or without sign, which must be surrounded by parentheses. Thus meters squared may be indicated by $m^{* *}(2)$, $m^{* *}+2, m+2, m 2, m^{\wedge} 2, m^{\wedge}(+2)$, etc. and per meter cubed may be indicated by $m^{* *}-3, m-3, m^{\wedge}(-3), / m 3$, and so forth. Meters to the three-halves power may be indicated by $m(1.5), m^{\wedge}(1.5)$, $m * *(1.5), m(3 / 2), m^{* *}(3 / 2)$, and $m^{\wedge}(3 / 2)$, but not by $m^{\wedge} 3 / 2$ or $\mathrm{m} 1.5$.

\subsubsection{Units in comment fields}

If the units of the keyword value are specified in the comment of the header keyword, it is recommended that the units string be enclosed in square brackets (i.e., enclosed by "[" and "]") at the beginning of the comment field, separated from the slash (" $/$ ") comment field delimiter by a single space character. An example, using a non-standard keyword, is

EXPTIME $=1200 . /$ [s] exposure time in seconds This widespread, but optional, practice suggests that square brackets should be used in comment fields only for this purpose. Nonetheless, software should not depend on units being expressed in this fashion within a keyword comment, and software should not depend on any string within square brackets in a comment field containing a proper units string.

\subsection{Keywords}

\subsubsection{Mandatory keywords}

Mandatory keywords are required in every HDU as described in the remainder of this subsection. They must be used only as described in this standard. Values of the mandatory keywords must be written in fixed-format.

4.4.1.1. Primary header. The SIMPLE keyword is required to be the first keyword in the primary header of all FITS files. The primary header must contain the other mandatory keywords shown in Table 7 in the order given. Other keywords must not intervene between the SIMPLE keyword and the last NAXISn keyword.

SIMPLE keyword. The value field shall contain a logical constant with the value $\mathrm{T}$ if the file conforms to this standard. This keyword is mandatory for the primary header and must not appear in extension headers ${ }^{6}$. A value of $F$ signifies that the file does not conform to this standard.

BITPIX keyword. The value field shall contain an integer. The absolute value is used in computing the sizes of data structures. It shall specify the number of bits that represent a data value in the associated data array. The only valid values of BITPIX are given in Table 8. Writers of FITS arrays should select a BITPIX data type appropriate to the form, range of values, and accuracy of the data in the array.

NAXIS keyword. The value field shall contain a non-negative integer no greater than 999 representing the number of axes in the associated data array. A value of zero signifies that no data follow the header in the HDU.

NAXISn keywords. The NAXISn keywords must be present for all values $n=1, \ldots$ NAXIS, in increasing order of $n$, and for no other values of $n$. The value field of this indexed keyword shall contain a non-negative integer representing the number of elements along axis $n$ of a data array. A value of zero for any of the NAXISn signifies that no data follow the header in the HDU (however, the random groups structure described in Sect. 6 has NAXIS1 $=0$, but will have data following the header if the other NAXISn keywords are non-zero). If NAXIS is equal to 0 , there shall not be any NAXISn keywords.

END keyword. This keyword has no associated value. Bytes 9 through 80 shall be filled with ASCII spaces (decimal 32 or hexadecimal 20). The END keyword marks the logical end of the

${ }^{6}$ This requirement differs from the wording in the original FITS papers. See Appendix H. 
Table 4. Additional allowed units.

\begin{tabular}{|c|c|c|c|c|}
\hline Quantity & & Unit & Meaning & Notes \\
\hline \multirow{4}{*}{\multicolumn{2}{|c|}{ plane angle }} & deg & degree of arc & $\pi / 180 \mathrm{rad}$ \\
\hline & & arcmin & minute of arc & $1 / 60 \mathrm{deg}$ \\
\hline & & $\operatorname{arcsec}$ & second of arc & $1 / 3600 \mathrm{deg}$ \\
\hline & & mas & milli-second of arc & $1 / 3600000 \mathrm{deg}$ \\
\hline \multirow[t]{5}{*}{ time } & & $\min$ & minute & $60 \mathrm{~s}$ \\
\hline & & $\mathrm{h}$ & hour & $60 \mathrm{~min}=3600 \mathrm{~s}$ \\
\hline & & $\mathrm{d}$ & day & $86400 \mathrm{~s}$ \\
\hline & $\dagger$ & $\mathrm{a}$ & year (Julian) & $31557600 \mathrm{~s}$ (365.25 d), peta a (Pa) forbidden \\
\hline & $\dagger$ & yr & year (Julian) & $\mathrm{a}$ is IAU-style \\
\hline \multirow[t]{3}{*}{ energy* } & $\dagger$ & $\mathrm{eV}$ & electron volt & $1.6021765 \times 10^{-19} \mathrm{~J}$ \\
\hline & $\ddagger$ & erg & erg & $10^{-7} \mathrm{~J}$ \\
\hline & & Ry & rydberg & $\frac{1}{2}\left(\frac{2 \pi e^{2}}{h c}\right)^{2} m_{e} c^{2}=13.605692 \mathrm{eV}$ \\
\hline \multirow[t]{2}{*}{ mass ${ }^{*}$} & & solMass & solar mass & $1.9891 \times 10^{30} \mathrm{~kg}$ \\
\hline & & $\mathrm{u}$ & unified atomic mass unit & $1.6605387 \times 10^{-27} \mathrm{~kg}$ \\
\hline luminosity & & sollum & solar luminosity & $3.8268 \times 10^{26} \mathrm{~W}$ \\
\hline \multirow[t]{5}{*}{ length } & $\ddagger$ & Angstrom & angstrom & $10^{-10} \mathrm{~m}$ \\
\hline & & solRad & solar radius & $6.9599 \times 10^{8} \mathrm{~m}$ \\
\hline & & $\mathrm{AU}$ & astronomical unit & $1.49598 \times 10^{11} \mathrm{~m}$ \\
\hline & & lyr & light year & $9.460730 \times 10^{15} \mathrm{~m}$ \\
\hline & $\dagger$ & pc & parsec & $3.0857 \times 10^{16} \mathrm{~m}$ \\
\hline \multirow[t]{2}{*}{ events } & & count & count & \\
\hline & & $\begin{array}{l}\text { ct } \\
\text { photon } \\
\text { ph }\end{array}$ & $\begin{array}{l}\text { count } \\
\text { photon } \\
\text { photon }\end{array}$ & \\
\hline \multirow[t]{3}{*}{ flux density } & $\dagger$ & Jy & jansky & $10^{-26} \mathrm{~W} \mathrm{~m}^{-2} \mathrm{~Hz}^{-1}$ \\
\hline & $\dagger$ & mag & (stellar) magnitude & \\
\hline & $\dagger$ & $\mathrm{R}$ & rayleigh & $10^{10} /(4 \pi)$ photons $\mathrm{m}^{-2} \mathrm{~s}^{-1} \mathrm{sr}^{-1}$ \\
\hline \multirow{3}{*}{$\begin{array}{l}\text { magnetic field } \\
\text { area }\end{array}$} & $\dagger$ & G & gauss & $10^{-4} \mathrm{~T}$ \\
\hline & & pixel & (image/detector) pixel & \\
\hline & $\dagger$ & barn & barn & $10^{-28} \mathrm{~m}^{2}$ \\
\hline \multicolumn{5}{|c|}{ Miscellaneous units } \\
\hline & & D & debye & $\frac{1}{3} \times 10^{-29}$ C.m \\
\hline & & $\begin{array}{l}\text { Sun } \\
\text { chan }\end{array}$ & $\begin{array}{l}\text { relative to Sun } \\
\text { (detector) channel }\end{array}$ & e.g., abundances \\
\hline & & bin & numerous applications & (including the 1-d analogue of pixel) \\
\hline & & voxel & 3-d analogue of pixel & \\
\hline & $\dagger$ & bit & binary information unit & \\
\hline & $\dagger$ & byte & (computer) byte & 8 bit \\
\hline & & $\begin{array}{l}\text { adu } \\
\text { beam }\end{array}$ & $\begin{array}{l}\text { Analog-to-digital converter } \\
\text { beam area of observation }\end{array}$ & as in $\mathrm{Jy} /$ beam \\
\hline
\end{tabular}

Notes. ${ }^{(\dagger)}$ Addition of prefixes for decimal multiples and submultiples are allowed. (\$) Deprecated in IAU Style Manual (McNally 1988) but still in use. ${ }^{(*)}$ Conversion factors from CODATA Internationally recommended values of the fundamental physical constants 2002 (http://physics . nist.gov/cuu/Constants/).

Table 5. Prefixes for multiples and submultiples.

\begin{tabular}{llcllc}
\hline \hline Submult & Prefix & Char & Mult & Prefix & Char \\
\hline $10^{-1}$ & deci & $\mathrm{d}$ & 10 & deca & $\mathrm{da}$ \\
$10^{-2}$ & centi & $\mathrm{c}$ & $10^{2}$ & hecto & $\mathrm{h}$ \\
$10^{-3}$ & milli & $\mathrm{m}$ & $10^{3}$ & kilo & $\mathrm{k}$ \\
$10^{-6}$ & micro & $\mathrm{u}$ & $10^{6}$ & mega & $\mathrm{M}$ \\
$10^{-9}$ & nano & $\mathrm{n}$ & $10^{9}$ & giga & $\mathrm{G}$ \\
$10^{-12}$ & pico & $\mathrm{p}$ & $10^{12}$ & tera & $\mathrm{T}$ \\
$10^{-15}$ & femto & $\mathrm{f}$ & $10^{15}$ & peta & $\mathrm{P}$ \\
$10^{-18}$ & atto & $\mathrm{a}$ & $10^{18}$ & exa & $\mathrm{E}$ \\
$10^{-21}$ & zepto & $\mathrm{z}$ & $10^{21}$ & zetta & $\mathrm{Z}$ \\
$10^{-24}$ & yocto & $\mathrm{y}$ & $10^{24}$ & yotta & $\mathrm{Y}$ \\
\hline
\end{tabular}

Table 6. Characters and strings allowed to denote mathematical operations.

\begin{tabular}{ll}
\hline \hline String & Meaning \\
\hline str1 str2 & Multiplication \\
str1*str2 & Multiplication \\
str1.str2 & Multiplication \\
str1/str2 & Division \\
str1**expr & Raised to the power expr \\
str1^expr & Raised to the power expr \\
str1expr & Raised to the power expr \\
$\log (\operatorname{str} 1)$ & Common Logarithm (to base 10) \\
$\ln (\operatorname{str} 1)$ & Natural Logarithm \\
exp(str1) & Exponential $\left(e^{\mathrm{str} 1}\right)$ \\
sqrt(str1) & Square root \\
\hline
\end{tabular}


Table 7. Mandatory keywords for primary header.

\begin{tabular}{cl}
\hline \hline$\#$ & Keyword \\
\hline 1 & SIMPLE $=\mathrm{T}$ \\
2 & BITPIX \\
3 & NAXIS \\
4 & NAXISn, $n=1, \ldots$, NAXIS \\
& $\vdots$ \\
& (other keywords) \\
& $\vdots$ \\
last & END \\
\hline
\end{tabular}

Table 8. Interpretation of valid BITPIX value.

\begin{aligned} & \hline \hline Value Data represented \\ & \hline 8 Character or unsigned binary integer \\ & 16 16-bit two's complement binary integer \\ & 32 32-bit two's complement binary integer \\ & 64 64-bit two's complement binary integer \\ &-32 IEEE single precision floating point \\ &-64 IEEE double precision floating point \\ & \hline\end{aligned}

header and must occur in the last 2880-byte FITS block of the header.

The total number of bits in the primary data array, exclusive of fill that is needed after the data to complete the last 2880-byte data block (Sect. 3.3.2), is given by the following expression:

$N_{\text {bits }}=\mid$ BITPIX $\mid \times($ NAXIS $1 \times$ NAXIS $2 \times \cdots \times$ NAXISm $)$,

where $N_{\text {bits }}$ must be non-negative and is the number of bits excluding fill, $m$ is the value of NAXIS, and BITPIX and the NAXISn represent the values associated with those keywords. Note that the random groups convention in the primary array has a more complicated structure whose size is given by Eq. (4). The header of the first FITS extension in the file, if present, shall start with the first FITS block following the data block that contains the last bit of the primary data array.

An example of a primary array header is shown in Table 9. In addition to the required keywords, it includes a few of the reserved keywords that are discussed in Sect. 4.4.2.

4.4.1.2. Conforming extensions. All conforming extensions, whether or not further specified in this standard, must use the keywords defined in Table 10 in the order specified. Other keywords must not intervene between the XTENSION keyword and the GCOUNT keyword. The BITPIX, NAXIS, NAXISn, and END keywords are defined in Sect. 4.4.1.

XTENSION keyword. The value field shall contain a character string giving the name of the extension type. This keyword is mandatory for an extension header and must not appear in the primary header ${ }^{7}$. To preclude conflict, extension type names must be registered with the IAUFWG. The current list of registered extensions is given in Appendix F. An up-to-date list is also maintained on the FITS Support Office web site.

\footnotetext{
7 This requirement differs from the wording in the original FITS papers. See Appendix H.
}

PCOUNT keyword. The value field shall contain an integer that shall be used in any way appropriate to define the data structure, consistent with Eq. (2). In IMAGE (Sect. 7.1) and TABLE (Sect. 7.2) extensions this keyword must have the value 0; in BINTABLE extensions (Sect. 7.3) it is used to specify the number of bytes that follow the main data table in the supplemental data area called the heap. This keyword is also used in the random groups structure (Sect. 6) to specify the number of parameters preceding each array in a group.

GCOUNT keyword. The value field shall contain an integer that shall be used in any way appropriate to define the data structure, consistent with Eq. (2). This keyword must have the value 1 in the IMAGE, TABLE and BINTABLE standard extensions defined in Sect. 7. This keyword is also used in the random groups structure (Sect. 6) to specify the number of random groups present.

The total number of bits in the extension data array (exclusive of fill that is needed after the data to complete the last 2880-byte data block) is given by the following expression:

$$
\begin{aligned}
N_{\text {bits }}= & \mid \text { BITPIX } \mid \times \text { GCOUNT } \\
& \times(\text { PCOUNT }+ \text { NAXIS } 1 \times \text { NAXIS } 2 \times \cdots \times \text { NAXISm }),
\end{aligned}
$$

where $N_{\text {bits }}$ must be non-negative and is the number of bits excluding fill; $m$ is the value of NAXIS; and BITPIX, GCOUNT, PCOUNT, and the NAXISn represent the values associated with those keywords. If $N_{\text {bits }}>0$, then the data array shall be contained in an integral number of 2880-byte FITS data blocks. The header of the next FITS extension in the file, if any, shall start with the first FITS block following the data block that contains the last bit of the current extension data array.

\subsubsection{Other reserved keywords}

The reserved keywords described below are optional, but if present in the header they must be used only as defined in this standard. They apply to any FITS structure with the meanings and restrictions defined below. Any FITS structure may further restrict the use of these keywords.

\subsubsection{General descriptive keywords}

DATE keyword. The value field shall contain a character string giving the date on which the HDU was created, in the form YYYY-MM-DD, or the date and time when the HDU was created, in the form YYYY-MY-DDThh:mm:ss[.sss...], where YYYY shall be the four-digit calendar year number, MM the two-digit month number with January given by 01 and December by 12 , and DD the two-digit day of the month. When both date and time are given, the literal $\mathrm{T}$ shall separate the date and time, hh shall be the two-digit hour in the day, mm the two-digit number of minutes after the hour, and ss[.sss...] the number of seconds (two digits followed by an optional fraction) after the minute. Default values must not be given to any portion of the date/time string, and leading zeros must not be omitted. The decimal part of the seconds field is optional and may be arbitrarily long, so long as it is consistent with the rules for value formats of Sect. 4.2.

The value of the DATE keyword shall always be expressed in UTC when in this format, for all data sets created on Earth.

The following format may appear on files written before January 1, 2000. The value field contains a character string giving the date on which the HDU was created, in the form 
Table 9. Example of a primary array header.

\begin{tabular}{lr}
\hline \hline Keyword records & \\
\hline SIMPLE $=$ & T/file does conform to FITS standard \\
BITPIX $=$ & $16 /$ number of bits per data pixel \\
NAXIS $=$ & $2 /$ number of data axes \\
NAXIS1 $=$ & $250 /$ length of data axis 1 \\
NAXIS2 $=$ & $300 /$ length of data axis 2 \\
OBJECT $=$ 'Cygnus X-1' & \\
DATE $=$ '2006-10-22' & \\
END & \\
\hline
\end{tabular}

Table 10. Mandatory keywords in conforming extensions.

\begin{tabular}{ll}
\hline \hline$\#$ & Keyword \\
\hline 1 & XTENSION \\
2 & BITPIX \\
3 & NAXIS \\
4 & NAXISn, $n=1, \ldots$, NAXIS \\
5 & PCOUNT \\
6 & GCOUNT \\
& $\vdots$ \\
& (other keywords) \\
& $\vdots$ \\
last & END \\
\hline
\end{tabular}

DD/MM/YY, where DD is the day of the month, MM the month number with January given by 01 and December by 12, and YY the last two digits of the year, the first two digits being understood to be 19. Specification of the date using Universal Time is recommended but not assumed.

When a newly created HDU is substantially a verbatim copy of another HDU, the value of the DATE keyword in the original HDU may be retained in the new HDU instead of updating the value to the current date and time.

ORIGIN keyword. The value field shall contain a character string identifying the organization or institution responsible for creating the FITS file.

EXTEND keyword. The value field shall contain a logical value indicating whether the FITS file is allowed to contain conforming extensions following the primary HDU. This keyword may only appear in the primary header and must not appear in an extension header. If the value field is T then there may be conforming extensions in the FITS file following the primary HDU. This keyword is only advisory, so its presence with a value $\mathrm{T}$ does not require that the FITS file contains extensions, nor does the absence of this keyword necessarily imply that the file does not contain extensions. Earlier versions of this standard stated that the EXTEND keyword must be present in the primary header if the file contained extensions, but this is no longer required.

BLOCKED keyword. This keyword is deprecated and should not be used in new FITS files. It is reserved primarily to prevent its use with other meanings. As previously defined, this keyword, if used, was required to appear only within the first 36 keywords in the primary header. Its presence with the required logical value of $\mathrm{T}$ advised that the physical block size of the FITS file on which it appears may be an integral multiple of the FITS block length and not necessarily equal to it.

\subsubsection{Keywords describing observations}

DATE-OBS keyword. The format of the value field for DATE-OBS keywords shall follow the prescriptions for the DATE keyword (Sect. 4.4.2). Either the four-digit year format or the two-digit year format may be used for observation dates from 1900 through 1999 although the four-digit format is recommended.

When the format with a four-digit year is used, the default interpretations for time should be UTC for dates beginning 1972-01-01 and UT before. Other date and time scales are permissible. The value of the DATE-OBS keyword shall be expressed in the principal time system or time scale of the HDU to which it belongs; if there is any chance of ambiguity, the choice should be clarified in comments. The value of DATE-OBS shall be assumed to refer to the start of an observation, unless another interpretation is clearly explained in the comment field. Explicit specification of the time scale is recommended. By default, times for TAI and times that run simultaneously with TAI, e.g., UTC and TT, will be assumed to be as measured at the detector (or, in practical cases, at the observatory). For coordinate times such as TCG, TCB and TDB, the default shall be to include light-time corrections to the associated spatial origin, namely the geocenter for TCG and the solar-system barycenter for the other two. Conventions may be developed that use other time systems. Appendix B of this document contains the Appendix to the agreement on a four-digit year, which discusses time systems in some detail.

When the value of DATE-OBS is expressed in the two-digit year form, allowed for files written before January 1, 2000 with a year in the range 1900-1999, there is no default assumption as to whether it refers to the start, middle or end of an observation.

DATExxxx keywords. The value fields for all keywords beginning with the string DATE whose value contains date, and $o p$ tionally time, information shall follow the prescriptions for the DATE-OBS keyword.

TELESCOP keyword. The value field shall contain a character string identifying the telescope used to acquire the data associated with the header.

INSTRUME keyword. The value field shall contain a character string identifying the instrument used to acquire the data associated with the header. 
OBSERVER keyword. The value field shall contain a character string identifying who acquired the data associated with the header.

OBJECT keyword. The value field shall contain a character string giving a name for the object observed.

\subsubsection{Bibliographic keywords}

AUTHOR keyword. The value field shall contain a character string identifying who compiled the information in the data associated with the header. This keyword is appropriate when the data originate in a published paper or are compiled from many sources.

REFERENC keyword. The value field shall contain a character string citing a reference where the data associated with the header are published. It is recommended that either the 19-digit bibliographic identifier ${ }^{8}$ used in the Astrophysics Data System bibliographic databases (http://adswww.harvard.edu/) or the Digital Object Identifier (http://doi . org) be included in the value string when available (e.g. "1994A\&AS . . 103 . .135A" or "doi : 10.1006/jmbi . 1998.2354").

4.4.2.4. Commentary keywords. These keywords provide commentary information about the contents or history of the FITS file and may occur any number of times in a header. These keywords shall have no associated value even if the value indicator characters " $=\sqcup "$ appear in bytes 9 and 10 (hence it is recommended that these keywords not contain the value indicator). Bytes 9 through 80 may contain any of the restricted set of ASCII text characters, decimal 32 through 126 (hexadecimal 20 through 7E).

COMMENT keyword. This keyword may be used to supply any comments regarding the FITS file.

HISTORY keyword. This keyword should be used to describe the history of steps and procedures associated with the processing of the associated data.

Keyword field is blank. This keyword may be used to supply any comments regarding the FITS file. It is frequently used for aesthetic purposes to provide a break between groups of related keywords in the header.

4.4.2.5. Keywords that describe arrays. These keywords are used to describe the contents of an array, either in the primary array, in an image extension (Sect. 7.1), or in a series of random groups (Sect. 6). They are optional, but if they appear in the header describing an array or groups, they must be used as defined in this section of this standard. They shall not be used in headers describing other structures unless the meaning is the same as defined here.

\footnotetext{
8 This bibliographic convention (Schmitz et al. 1995) was initially developed for use within NED (NASA/IPAC Extragalactic Database) and SIMBAD (operated at CDS, Strasbourg, France).
}

BSCALE keyword. This keyword shall be used, along with the BZERO keyword, to linearly scale the array pixel values (i.e., the actual values stored in the FITS file) to transform them into the physical values that they represent using Eq. (3).

physical_value $=$ BZERO + BSCALE $\times$ array_value.

The value field shall contain a floating-point number representing the coefficient of the linear term in the scaling equation, the ratio of physical value to array value at zero offset. The default value for this keyword is 1.0. Before support for IEEE floatingpoint data types was added to FITS (Wells \& Grosbøl 1990), this technique of linearly scaling integer values was the only way to represent the full range of floating-point values in a FITS array. This linear scaling technique is still commonly used to reduce the size of the data array by a factor of two by representing 32-bit floating-point physical values as 16-bit scaled integers.

BZERO keyword. This keyword shall be used, along with the BSCALE keyword, to linearly scale the array pixel values (i.e., the actual values stored in the FITS file) to transform them into the physical values that they represent using Eq. (3). The value field shall contain a floating-point number representing the physical value corresponding to an array value of zero. The default value for this keyword is 0.0 .

Besides its use in representing floating-point values as scaled integers (see the description of the BSCALE keyword), the BZERO keyword is also used when storing unsigned integer values in the FITS array. In this special case the BSCALE keyword shall have the default value of 1.0, and the BZERO keyword shall have one of the integer values shown in Table 11.

Since the FITS format does not support a native unsigned integer data type (except for the unsigned 8-bit byte data type), the unsigned values are stored in the FITS array as native signed integers with the appropriate integer offset specified by the BZERO keyword value shown in the table. For the byte data type, the converse technique can be used to store signed byte values as native unsigned values with the negative BZERO offset. In each case, the physical value is computed by adding the offset specified by the BZERO keyword to the native data type value that is stored in the FITS file 9 .

BUNIT keyword. The value field shall contain a character string describing the physical units in which the quantities in the array, after application of BSCALE and BZERO, are expressed. These units must follow the prescriptions of Sect. 4.3.

BLANK keyword. This keyword shall be used only in headers with positive values of BITPIX (i.e., in arrays with integer data). Bytes 1 through 8 contain the string "BLANK $\sqcup \sqcup$ " (ASCII spaces in bytes 6 through 8 ). The value field shall contain an integer that specifies the value that is used within the integer array to represent pixels that have an undefined physical value.

If the BSCALE and BZERO keywords do not have the default values of 1.0 and 0.0 , respectively, then the value of the BLANK keyword must equal the actual value in the FITS data array that is used to represent an undefined pixel and not the

\footnotetext{
9 A more computationally efficient method of adding or subtracting the BZERO values is to simply flip the most significant bit of the binary value. For example, using 8-bit integers, the decimal value $248 \mathrm{mi}$ nus the BZERO value of 128 equals 120 . The binary representation of 248 is 11111000 . Flipping the most significant bit gives the binary value 01111000 , which is equal to decimal 120.
} 
Table 11. Usage of BZERO to represent non-default integer data types.

\begin{tabular}{rccrl}
\hline \hline BITPIX & $\begin{array}{c}\text { Native } \\
\text { data type }\end{array}$ & $\begin{array}{c}\text { Physical } \\
\text { data type }\end{array}$ & BZERO & \\
\hline 8 & unsigned & signed byte & -128 & $\left(-2^{7}\right)$ \\
16 & signed & unsigned 16-bit & 32768 & $\left(2^{15}\right)$ \\
32 & signed & unsigned 32-bit & 2147483648 & $\left(2^{31}\right)$ \\
64 & signed & unsigned 64-bit & 9223372036854775808 & $\left(2^{63}\right)$ \\
\hline
\end{tabular}

corresponding physical value (computed from Eq. (3)). To cite a specific, common example, unsigned 16-bit integers are represented in a signed integer FITS array (with BITPIX = 16) by setting BZERO $=32768$ and BSCALE $=1$. If it is desired to use pixels that have an unsigned value (i.e., the physical value) equal to 0 to represent undefined pixels in the array, then the BLANK keyword must be set to the value -32768 because that is the actual value of the undefined pixels in the FITS array.

DATAMAX keyword. The value field shall always contain a floating-point number, regardless of the value of BITPIX. This number shall give the maximum valid physical value represented by the array (from Eq. (3)), exclusive of any IEEE special values.

DATAMIN keyword. The value field shall always contain a floating-point number, regardless of the value of BITPIX. This number shall give the minimum valid physical value represented by the array (from Eq. (3)), exclusive of any IEEE special values.

WCS keywords. An extensive set of keywords have been defined to describe the world coordinates associated with an array. These keywords are discussed separately in Sect. 8.

4.4.2.6. Extension keywords. Although these keywords were originally defined for use within the header of a conforming extension, they also may appear in the primary header with an analogous meaning. If these keywords are present, it is recommended that they have a unique combination of values in each HDU of the FITS file.

EXTNAME keyword. The value field shall contain a character string to be used to distinguish among different extensions of the same type, i.e., with the same value of XTENSION, in a FITS file. Within this context, the primary array should be considered as equivalent to an IMAGE extension.

EXTVER keyword. The value field shall contain an integer to be used to distinguish among different extensions in a FITS file with the same type and name, i.e., the same values for XTENSION and EXTNAME. The values need not start with 1 for the first extension with a particular value of EXTNAME and need not be in sequence for subsequent values. If the EXTVER keyword is absent, the file should be treated as if the value were 1 .

EXTLEVEL keyword. The value field shall contain an integer specifying the level in a hierarchy of extension levels of the extension header containing it. The value shall be 1 for the highest level; levels with a higher value of this keyword shall be subordinate to levels with a lower value. If the EXTLEVEL keyword is absent, the file should be treated as if the value were 1 .

\subsubsection{Additional keywords}

New keywords may be devised in addition to those described in this standard, so long as they are consistent with the generalized rules for keywords and do not conflict with mandatory or reserved keywords. Any keyword that refers to or depends upon the existence of other specific HDUs in the same or other files should be used with caution because the persistence of those HDUs cannot always be guaranteed.

\section{Data representation}

Primary and extension data shall be represented in one of the formats described in this section. FITS data shall be interpreted to be a byte stream. Bytes are in big-endian order of decreasing significance. The byte that includes the sign bit shall be first, and the byte that has the ones bit shall be last.

\subsection{Characters}

Each character shall be represented by one byte. A character shall be represented by its 7-bit ASCII (ANSI 1977) code in the low order seven bits in the byte. The high-order bit shall be zero.

\subsection{Integers}

\subsubsection{Eight-bit}

Eight-bit integers shall be unsigned binary integers, contained in one byte with decimal values ranging from 0 to 255 .

\subsubsection{Sixteen-bit}

Sixteen-bit integers shall be two's complement signed binary integers, contained in two bytes with decimal values ranging from -32768 to +32767 .

\subsubsection{Thirty-two-bit}

Thirty-two-bit integers shall be two's complement signed binary integers, contained in four bytes with decimal values ranging from -2147483648 to +2147483647 .

\subsubsection{Sixty-four-bit}

Sixty-four-bit integers shall be two's complement signed binary integers, contained in eight bytes with decimal values ranging from -9223372036854775808 to +9223372036854775807 .

\subsubsection{Unsigned integers}

The FITS format does not support a native unsigned integer data type (except for the unsigned 8-bit byte data type) therefore unsigned 16-bit, 32-bit, or 64-bit binary integers cannot be stored 
directly in a FITS data array. Instead, the appropriate offset must be applied to the unsigned integer to shift the value into the range of the corresponding signed integer, which is then stored in the FITS file. The BZERO keyword shall record the amount of the offset needed to restore the original unsigned value. The BSCALE keyword shall have the default value of 1.0 in this case, and the appropriate BZERO value, as a function of BITPIX, is specified in Table 11.

This same technique must be used when storing unsigned integers in a binary table column of signed integers (Sect. 7.3.2). In this case the TSCALn keyword (analogous to BSCALE) shall have the default value of 1.0, and the appropriate TZEROn value (analogous to BZERO) is specified in Table 19.

\subsection{IEEE-754 floating point}

Transmission of 32- and 64-bit floating-point data within the FITS format shall use the ANSI/IEEE-754 standard (IEEE 1985). BITPIX $=-32$ and BITPIX $=-64$ signify 32- and 64-bit IEEE floating-point numbers, respectively; the absolute value of BITPIX is used for computing the sizes of data structures. The full IEEE set of number forms is allowed for FITS interchange, including all special values.

The BLANK keyword should not be used when BITPIX $=-32$ or -64 ; rather, the IEEE NaN should be used to represent an undefined value. Use of the BSCALE and BZERO keywords is not recommended.

Appendix E has additional details on the IEEE format.

\section{Random groups structure}

The random groups structure allows a collection of "groups", where a group consists of a subarray of data and a set of associated parameter values, to be stored within the FITS primary data array. Random groups have been used almost exclusively for applications in radio interferometry; outside this field, there is little support for reading or writing data in this format. Other than the existing use for radio interferometry data, the random groups structure is deprecated and should not be further used. For other applications, the binary table extension (Sect. 7.3) provides a more extensible and better documented way of associating groups of data within a single data structure.

\subsection{Keywords}

\subsubsection{Mandatory keywords}

The SIMPLE keyword is required to be the first keyword in the primary header of all FITS files, including those with random groups records. If the random groups format records follow the primary header, the keyword records of the primary header must use the keywords defined in Table 12 in the order specified. No other keywords may intervene between the SIMPLE keyword and the last NAXISn keyword.

SIMPLE keyword. The keyword record containing this keyword is structured in the same way as if a primary data array were present (Sect. 4.4.1).

BITPIX keyword. The keyword record containing this keyword is structured as prescribed in Sect. 4.4.1.
Table 12. Mandatory keywords in primary header preceding random groups.

\begin{tabular}{ll}
\hline \hline$\#$ & Keyword \\
\hline 1 & SIMPLE $=\mathrm{T}$ \\
2 & BITPIX \\
3 & NAXIS \\
4 & NAXIS1 $=0$ \\
5 & NAXISn, $n=2, \ldots$, value of NAXIS \\
& $\vdots$ \\
& (other keywords, which must include ....) \\
& GROUPS = T \\
& PCOUNT \\
& GCOUNT \\
& $\vdots$ \\
last & END \\
\hline
\end{tabular}

NAXIS keyword. The value field shall contain an integer ranging from 1 to 999, representing one more than the number of axes in each data array.

NAXIS1 keyword. The value field shall contain the integer $\theta$, a signature of random groups format indicating that there is no primary data array.

NAXISn keywords ( $n=2, \ldots$, value of NAXIS) The NAXISn keywords must be present for all values $n=2, \ldots$, NAXIS, in increasing order of $n$, and for no larger values of $n$. The value field shall contain an integer, representing the number of positions along axis $n-1$ of the data array in each group.

GROUPS keyword. The value field shall contain the logical constant $\mathrm{T}$. The value $\mathrm{T}$ associated with this keyword implies that random groups records are present.

PCOUNT keyword. The value field shall contain an integer equal to the number of parameters preceding each array in a group.

GCOUNT keyword. The value field shall contain an integer equal to the number of random groups present.

END keyword. This keyword has no associated value. Bytes 9 through 80 shall contain ASCII spaces (decimal 32 or hexadecimal 20).

The total number of bits in the random groups records exclusive of the fill described in Sect. 6.2 is given by the following expression:

$$
\begin{aligned}
N_{\text {bits }}= & \mid \text { BITPIX } \mid \times \text { GCOUNT } \\
& \times(\text { PCOUNT }+ \text { NAXIS } 2 \times \text { NAXIS } 3 \times \cdots \times \text { NAXISm }),
\end{aligned}
$$

where $N_{\text {bits }}$ is non-negative and the number of bits excluding fill; $\mathrm{m}$ is the value of NAXIS; and BITPIX, GCOUNT, PCOUNT, and the NAXISn represent the values associated with those keywords.

\subsubsection{Reserved keywords}

PTYPEn keywords. The value field shall contain a character string giving the name of parameter $n$. If the PTYPEn keywords 
for more than one value of $n$ have the same associated name in the value field, then the data value for the parameter of that name is to be obtained by adding the derived data values of the corresponding parameters. This rule provides a mechanism by which a random parameter may have more precision than the accompanying data array elements; for example, by summing two 16-bit values with the first scaled relative to the other such that the sum forms a number of up to 32-bit precision.

PSCALn keywords. This keyword shall be used, along with the PZEROn keyword, when the $n$th FITS group parameter value is not the true physical value, to transform the group parameter value to the true physical values it represents, using Eq. (5). The value field shall contain a floating-point number representing the coefficient of the linear term in Eq. (5), the scaling factor between true values and group parameter values at zero offset. The default value for this keyword is 1.0.

PZEROn keywords. This keyword shall be used, along with the PSCALn keyword, when the $n$th FITS group parameter value is not the true physical value, to transform the group parameter value to the physical value. The value field shall contain a floating-point number, representing the true value corresponding to a group parameter value of zero. The default value for this keyword is 0.0. The transformation equation is as follows:

physical_value $=$ PZEROn + PSCALn $\times$ group_parm_value.

\subsection{Data sequence}

Random groups data shall consist of a set of groups. The number of groups shall be specified by the GCOUNT keyword in the associated header. Each group shall consist of the number of parameters specified by the PCOUNT keyword followed by an array with the number of elements $N_{\text {elem }}$ given by the following expression:

$N_{\text {elem }}=($ NAXIS $2 \times$ NAXIS $3 \times \cdots \times$ NAXISm $)$,

where $N_{\text {elem }}$ is the number of elements in the data array in a group, $m$ is the value of NAXIS, and the NAXISn represent the values associated with those keywords.

The first parameter of the first group shall appear in the first location of the first data block. The first element of each array shall immediately follow the last parameter associated with that group. The first parameter of any subsequent group shall immediately follow the last element of the array of the previous group. The arrays shall be organized internally in the same way as an ordinary primary data array. If the groups data do not fill the final data block, the remainder of the block shall be filled with zero values in the same way as a primary data array (Sect. 3.3.2). If random groups records are present, there shall be no primary data array.

\subsection{Data representation}

Permissible data representations are those listed in Sect. 5. Parameters and elements of associated data arrays shall have the same representation. If more precision is required for an associated parameter than for an element of a data array, the parameter shall be divided into two or more addends, represented by the same value for the PTYPEn keyword. The value shall be the sum of the physical values, which may have been obtained
Table 13. Mandatory keywords in image extensions.

\begin{tabular}{cl}
\hline \hline$\#$ & Keyword \\
\hline 1 & XTENSION=॰' IMAGE \\
2 & BITPIX \\
3 & NAXIS \\
4 & NAXISn, $n=1, \ldots$, NAXIS \\
5 & PCOUNT $=0$ \\
6 & GCOUNT $=1$ \\
& $\vdots$ \\
& $($ other keywords ...) \\
& $\vdots$ \\
last & END \\
\hline
\end{tabular}

from the group parameter values using the PSCALn and PZEROn keywords.

\section{Standard extensions}

A standard extension is a conforming extension whose organization and content are completely specified in this standard. The specifications for the 3 currently defined standard extensions, namely,

1. "IMAGE" extensions;

2. "TABLE" ASCII table extensions; and

3. "BINTABLE” binary table extensions;

are given in the following sections. A list of other conforming extensions is given in Appendix F.

\subsection{Image extension}

The FITS image extension is nearly identical in structure to the the primary HDU and is used to store an array of data. Multiple image extensions can be used to store any number of arrays in a single FITS file. The first keyword in an image extension shall be XTENSION=レ' IMAGE $\sqcup \sqcup$, '.

\subsubsection{Mandatory keywords}

The XTENSION keyword is required to be the first keyword of all image extensions. The keyword records in the header of an image extension must use the keywords defined in Table 13 in the order specified. No other keywords may intervene between the XTENSION and GCOUNT keywords.

XTENSION keyword. The value field shall contain the character string 'IMAGE

BITPIX keyword. The value field shall contain an integer. The absolute value is used in computing the sizes of data structures. It shall specify the number of bits that represent a data value. The only valid values of BITPIX are given in Table 8 . Writers of IMAGE extensions should select a BITPIX data type appropriate to the form, range of values, and accuracy of the data in the array.

NAXIS keyword. The value field shall contain a non-negative integer no greater than 999, representing the number of axes in the associated data array. If the value is zero then the image extension shall not have any data blocks following the header. 
Table 14. Mandatory keywords in ASCII table extensions.

\begin{tabular}{|c|c|}
\hline$\#$ & Keyword \\
\hline 1 & XTENSION=ப' TABLE ${ }_{\sqcup \sqcup \sqcup}$ ' \\
\hline 2 & BITPIX $=8$ \\
\hline 3 & NAXIS $=2$ \\
\hline 4 & NAXIS1 \\
\hline 5 & NAXIS2 \\
\hline 6 & PCOUNT $=0$ \\
\hline 7 & GCOUNT $=1$ \\
\hline \multirow[t]{7}{*}{8} & TFIELDS \\
\hline & : \\
\hline & (other keywords, including (if TFIELDS is not zero) ...) \\
\hline & TTYPEn, $n=1,2, \ldots, k$ where $k$ is the value of TFIELDS (Recommended) \\
\hline & TBCOLn, $n=1,2, \ldots, k$ where $k$ is the value of TFIELDS (Required) \\
\hline & TFORMn, $n=1,2, \ldots, k$ where $k$ is the value of TFIELDS (Required) \\
\hline & ( \\
\hline last & END \\
\hline
\end{tabular}

NAXISn keywords. The NAXISn keywords must be present for all values $n=1, \ldots$ NAXIS, in increasing order of $n$, and for no other values of $n$. The value field of this indexed keyword shall contain a non-negative integer, representing the number of elements along axis $n$ of a data array. If the value of any of the NAXISn keywords is zero, then the image extension shall not have any data blocks following the header. If NAXIS is equal to 0 , there should not be any NAXISn keywords.

PCOUNT keyword. The value field shall contain the integer $\theta$.

GCOUNT keyword. The value field shall contain the integer 1; each image extension contains a single array.

END keyword. This keyword has no associated value. Bytes 9 through 80 shall be filled with ASCII spaces (decimal 32 or hexadecimal 20).

\subsubsection{Other reserved keywords}

The reserved keywords defined in Sect. 4.4.2 (except for EXTEND and BLOCKED) may appear in an image extension header. The keywords must be used as defined in that section.

\subsubsection{Data sequence}

The data format shall be identical to that of a primary data array as described in Sect. 3.3.2.

\subsection{The ASCII table extension}

The ASCII table extension provides a means of storing catalogs and tables of astronomical data in FITS format. Each row of the table consists of a fixed-length sequence of ASCII characters divided into fields that correspond to the columns in the table. The first keyword in an ASCII table extension shall be XTENSION= $=$ ' TABLE ${ }_{\sqcup \sqcup \sqcup}$ '.

\subsubsection{Mandatory keywords}

The header of an ASCII table extension must use the keywords defined in Table 14. The first keyword must be XTENSION; the seven keywords following XTENSION (BITPIX ... TFIELDS) must be in the order specified with no intervening keywords.

XTENSION keyword. The value field shall contain the character

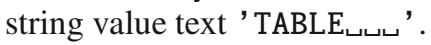

BITPIX keyword. The value field shall contain the integer 8, denoting that the array contains ASCII characters.

NAXIS keyword. The value field shall contain the integer 2, denoting that the included data array is two-dimensional: rows and columns.

NAXIS1 keyword. The value field shall contain a non-negative integer, giving the number of ASCII characters in each row of the table. This includes all the characters in the defined fields plus any characters that are not included in any field.

NAXIS2 keyword. The value field shall contain a non-negative integer, giving the number of rows in the table.

PCOUNT keyword. The value field shall contain the integer $\Theta$.

GCOUNT keyword. The value field shall contain the integer 1; the data blocks contain a single table.

TFIELDS keyword. The value field shall contain a non-negative integer representing the number of fields in each row. The maximum permissible value is 999 .

TBCOLn keywords. The TBCOLn keywords must be present for all values $n=1, \ldots$, TFIELDS and for no other values of $n$. The value field of this indexed keyword shall contain an integer specifying the column in which field $n$ starts. The first column of a row is numbered 1 . 
Table 15. Valid TFORMn format values in TABLE extensions.

\begin{aligned} & \hline \hline Field value Data type \\ & \hline Aw Character \\ & Iw Decimal integer \\ & Fw.d Floating-point, fixed decimal notation \\ & Ew.d Floating-point, exponential notation \\ & Dw.d Floating-point, exponential notation \\ & \hline\end{aligned}

Notes. $w$ is the width in characters of the field and $d$ is the number of digits to the right of the decimal.

TFORMn keywords. The TFORMn keywords must be present for all values $n=1, \ldots$, TFIELDS and for no other values of $n$. The value field of this indexed keyword shall contain a character string describing the format in which field $n$ is encoded. Only the formats in Table 15, interpreted as Fortran (ISO 2004) input formats and discussed in more detail in Sect. 7.2.5, are permitted for encoding. Format codes must be specified in upper case. Other format editing codes common to Fortran such as repetition, positional editing, scaling, and field termination are not permitted. All values in numeric fields have a number base of ten (i.e., they are decimal); binary, octal, hexadecimal, and other representations are not permitted. The TDISPn keyword, defined in Sect. 7.2.2, may be used to recommend that a decimal integer value in an ASCII table be displayed as the equivalent binary, octal, or hexadecimal value.

END keyword. This keyword has no associated value. Bytes 9 through 80 shall contain ASCII spaces (decimal 32 or hexadecimal 20).

\subsubsection{Other reserved keywords}

In addition to the reserved keywords defined in Sect. 4.4 .2 (except for EXTEND and BLOCKED), the following other reserved keywords may be used to describe the structure of an ASCII table data array. They are optional, but if they appear within an ASCII table extension header, they must be used as defined in this section of this standard.

TTYPEn keywords. The value field for this indexed keyword shall contain a character string giving the name of field $n$. It is strongly recommended that every field of the table be assigned a unique, case-insensitive name with this keyword, and it is recommended that the character string be composed only of upper and lower case letters, digits, and the underscore ("_,", decimal 95, hexadecimal $5 \mathrm{~F}$ ) character. Use of other characters is not recommended because it may be difficult to map the column names into variables in some languages (e.g., any hyphens, "*” or "+" characters in the name may be confused with mathematical operators). String comparisons with the TTYPEn keyword values should not be case sensitive (e.g., "TIME" and "Time" should be interpreted as the same name).

TUNITn keywords. The value field shall contain a character string describing the physical units in which the quantity in field $n$, after any application of TSCALn and TZEROn, is expressed. Units must follow the prescriptions in Sect. 4.3.
TSCALn keywords. This indexed keyword shall be used, along with the TZEROn keyword, to linearly scale the values in the table field $n$ to transform them into the physical values that they represent using Eq. (7). The value field shall contain a floatingpoint number representing the coefficient of the linear term in the scaling equation. The default value for this keyword is 1.0. This keyword must not be used for A-format fields.

The transformation equation used to compute a true physical value from the quantity in field $n$ is

physical_value $=$ TZEROn + TSCALn $\times$ field_value

where field_value is the value that is actually stored in that table field in the FITS file.

TZEROn keywords. This indexed keyword shall be used, along with the TSCALn keyword, to linearly scale the values in the table field $n$ to transform them into the physical values that they represent using Eq. (7). The value field shall contain a floatingpoint number representing the physical value corresponding to an array value of zero. The default value for this keyword is 0.0 . This keyword must not be used for A-format fields.

TNULLn keywords. The value field for this indexed keyword shall contain the character string that represents an undefined value for field $n$. The string is implicitly space filled to the width of the field.

TDISPn keywords. The value field of this indexed keyword shall contain a character string describing the format recommended for displaying an ASCII text representation of of the contents of field $n$. This keyword overrides the default display format given by the TFORMn keyword. If the table value has been scaled, the physical value, derived using Eq. (7), shall be displayed. All elements in a field shall be displayed with a single, repeated format. Only the format codes in Table 16, interpreted as Fortran (ISO 2004) output formats, and discussed in more detail in Sect. 7.3.4, are permitted for encoding. The format codes must be specified in upper case. If the Bw.m, Ow.m, and $\mathrm{Zw} . \mathrm{m}$ formats are not readily available to the reader, the Iw.m display format may be used instead, and if the ENw . d and ESw . d formats are not available, Ew. d may be used.

\subsubsection{Data sequence}

The table is constructed from a two-dimensional array of ASCII characters. The row length and the number of rows shall be those specified, respectively, by the NAXIS1 and NAXIS2 keywords of the associated header. The number of characters in a row and the number of rows in the table shall determine the size of the character array. Every row in the array shall have the same number of characters. The first character of the first row shall be at the start of the data block immediately following the last header block. The first character of subsequent rows shall follow immediately the character at the end of the previous row, independent of the FITS block structure. The positions in the last data block after the last character of the last row of the table shall be filled with ASCII spaces.

\subsubsection{Fields}

Each row in the array shall consist of a sequence of from 0 to 999 fields, as specified by the TFIELDS keyword, with one entry 
Table 16. Valid TDISPn format values in TABLE extensions.

\begin{aligned} & \hline \hline Field value Data type \\ & \hline Aw Character \\ & Iw.m Integer \\ & Bw.m Binary, integers only \\ & Ow.m Octal, integers only \\ & Zw.m Hexadecimal, integers only \\ & Fw.d Floating-point, fixed decimal notation \\ & Ew.dEe Floating-point, exponential notation \\ & ENw.d Engineering; E format with exponent multiple of three \\ & ESw.d Scientific; same as EN but non-zero leading digit if not zero \\ & Gw.dEe General; appears as F if significance not lost, else E. \\ & Dw.dEe Floating-point, exponential notation \\ & \hline\end{aligned}

Notes. $w$ is the width in characters of displayed values, $m$ is the minimum number of digits displayed, $d$ is the number of digits to right of decimal, and e is number of digits in exponent. The . $\mathrm{m}$ and Ee fields are optional.

in each field. For every field, the Fortran (ISO 2004) format of the information contained (given by the TFORMn keyword), the location in the row of the beginning of the field (given by the TBCOLn keyword), and (optionally, but strongly recommended) the field name (given by the TTYPEn keyword), shall be specified in the associated header. The location and format of fields shall be the same for every row. Fields may overlap, but this usage is not recommended. Only a limited set of ASCII character values may appear within any field, depending on the field type as specified below. There may be characters in a table row that are not included in any field, (e.g., between fields, or before the first field or after the last field). Any 7-bit ASCII character may occur in characters of a table row that are not included in a defined field. A common convention is to include a space character between each field for added legibility if the table row is displayed verbatim. It is also permissible to add control characters, such as a carriage return or line feed character, following the last field in each row as a way of formatting the table if it is printed or displayed by a text editing program.

\subsubsection{Entries}

All data in an ASCII table extension field shall be ASCII text in a format that conforms to the rules for fixed field input in Fortran (ISO 2004) format, as described below. The only possible formats shall be those specified in Table 15 . If values of -0 and +0 need to be distinguished, then the sign character should appear in a separate field in character format. TNULLn keywords may be used to specify a character string that represents an undefined value in each field. The characters representing an undefined value may differ from field to field but must be the same within a field. Writers of ASCII tables should select a format for each field that is appropriate to the form, range of values, and accuracy of the data in that field. This standard does not impose an upper limit on the number of digits of precision, nor any limit on the range of numeric values. Software packages that read or write data according to this standard could be limited, however, in the range of values and exponents that are supported (e.g., to the range that can be represented by 32-bit or 64-bit binary numbers).

The value of each entry shall be interpreted as described in the following paragraphs.

Character fields. The value of a character-formatted (Aw) field is a character string of width $w$ containing the characters in columns TBCOL $n$ through TBCOL $n+\mathrm{w}-1$. The character string shall be composed of the restricted set of ASCII text characters with decimal values in the range 32 through 126 (hexadecimal 20 through 7E).

Integer fields. The value of an integer-formatted (Iw) field is a signed decimal integer contained in columns TBCOL $n$ through TBCOL $n+\mathrm{w}-1$ consisting of a single optional sign ("+" or "_") followed by one or more decimal digits (" 0 " through " 9 "). Nonsignificant space characters may precede and/or follow the integer value within the field. A blank field has value 0 . All characters other than leading and trailing spaces, a contiguous string of decimal digits, and a single leading sign character are forbidden.

Real fields. The value of a real-formatted field (Fw.d, Ew.d, Dw.d) is a real number determined from the w characters from columns TBCOL $n$ through TBCOL $n+\mathrm{w}-1$. The value is formed by:

1. discarding any trailing space characters in the field and rightjustifying the remaining characters;

2. interpreting the first non-space characters as a numeric string consisting of a single optional sign ("+" or "-") followed by one or more decimal digits (" 0 " through " 9 ") optionally containing a single decimal point ("."). The numeric string is terminated by the end of the right-justified field or by the occurrence of any character other than a decimal point (".") and the decimal integers ("0" through "9"). If the string contains no explicit decimal point, then the implicit decimal point is taken as immediately preceding the rightmost $d$ digits of the string, with leading zeros assumed if necessary. The use of implicit decimal points is deprecated and is strongly discouraged because of the possibility that FITS reading programs will misinterpret the data value. Therefore, real-formatted fields should always contain an explicit decimal point;

3. if the numeric string is terminated by a

(a) "+" or "-", interpreting the following string as an exponent in the form of a signed decimal integer; or

(b) "E", or "D", interpreting the following string as an exponent of the form E or D followed by an optionally signed decimal integer constant;

4. the exponent string, if present, is terminated by the end of the right-justified string;

5. characters other than those specified above, including embedded space characters, are forbidden. 
Table 17. Mandatory keywords in binary table extensions.

\begin{tabular}{|c|c|}
\hline \# & Keyword \\
\hline 1 & XTENSION=ப'BINTABLE' \\
\hline 2 & BITPIX $=8$ \\
\hline 3 & NAXIS $=2$ \\
\hline 4 & NAXIS1 \\
\hline 5 & NAXIS2 \\
\hline 6 & PCOUNT \\
\hline 7 & GCOUNT $=1$ \\
\hline \multirow[t]{6}{*}{8} & TFIELDS \\
\hline & $\vdots$ \\
\hline & (other keywords, including (if TFIELDS is not zero) ...) \\
\hline & TTYPEn, $n=1,2, \ldots, k$ where $k$ is the value of TFIELDS (Recommended) \\
\hline & TFORMn, $n=1,2, \ldots, k$ where $k$ is the value of TFIELDS (Required) \\
\hline & $\vdots$ \\
\hline last & END \\
\hline
\end{tabular}

The numeric value of the table field is then the value of the numeric string multiplied by ten (10) to the power of the exponent string, i.e., value $=$ numeric_string $\times 10^{\text {(exponent_string) }}$. The default exponent is zero and a blank field has value zero. There is no difference between the F, D, and E formats; the content of the string determines its interpretation. Numbers requiring more precision and/or range than the local computer can support may be represented. It is good form to specify a D format in TFORM $n$ for a column of an ASCII table when that column will contain numbers that cannot be accurately represented in 32-bit IEEE binary format (see Appendix E).

\subsection{Binary table extension}

The binary table extension is similar to the ASCII table in that it provides a means of storing catalogs and tables of astronomical data in FITS format, however, it offers more features and provides more-efficient data storage than ASCII tables. The numerical values in binary tables are stored in more-compact binary formats rather than coded into ASCII, and each field of a binary table can contain an array of values rather than a simple scalar as in ASCII tables. The first keyword in a binary table extension shall be XTENSION= 'BINTABLE'.

\subsubsection{Mandatory keywords}

The XTENSION keyword is the first keyword of all binary table extensions. The seven keywords following (BITPIX ... TFIELDS) must be in the order specified in Table 17, with no intervening keywords.

XTENSION keyword. The value field shall contain the character string 'BINTABLE'.

BITPIX keyword. The value field shall contain the integer 8, denoting that the array is an array of 8-bit bytes.

NAXIS keyword. The value field shall contain the integer 2, denoting that the included data array is two-dimensional: rows and columns.
NAXIS1 keyword. The value field shall contain a non-negative integer, giving the number of 8-bit bytes in each row of the table.

NAXIS2 keyword. The value field shall contain a non-negative integer, giving the number of rows in the table.

PCOUNT keyword. The value field shall contain the number of bytes that follow the table in the supplemental data area called the heap.

GCOUNT keyword. The value field shall contain the integer 1; the data blocks contain a single table.

TFIELDS keyword. The value field shall contain a non-negative integer representing the number of fields in each row. The maximum permissible value is 999 .

TFORMn keywords. The TFORMn keywords must be present for all values $n=1, \ldots$, TFIELDS and for no other values of $n$. The value field of this indexed keyword shall contain a character string of the form $r \mathrm{~T} a$. The repeat count $r$ is the ASCII representation of a non-negative integer specifying the number of elements in field $n$. The default value of $r$ is 1 ; the repeat count need not be present if it has the default value. A zero element count, indicating an empty field, is permitted. The data type T specifies the data type of the contents of field $n$. Only the data types in Table 18 are permitted. The format codes must be specified in upper case. For fields of type $\mathrm{P}$ or $\mathrm{Q}$, the only permitted repeat counts are 0 and 1 . The additional characters $a$ are optional and are not further defined in this standard. Table 18 lists the number of bytes each data type occupies in a table row. The first field of a row is numbered 1 . The total number of bytes $n_{\text {row }}$ in a table row is given by

$$
n_{\text {row }}=\sum_{i=1}^{\text {TFIELDS }} r_{i} b_{i}
$$

where $r_{i}$ is the repeat count for field $i, b_{i}$ is the number of bytes for the data type in field $i$, and TFIELDS is the value of that keyword, must equal the value of NAXIS1. 
Table 18. Valid TFORMn data types in BINTABLE extensions.

\begin{tabular}{ccr}
\hline \hline TFORMn value & Description & 8-bit Bytes \\
\hline L & Logical & 1 \\
X & Bit & $\dagger$ \\
B & Unsigned byte & 1 \\
I & 16-bit integer & 2 \\
J & 32-bit integer & 4 \\
K & 64-bit integer & 8 \\
A & Character & 1 \\
E & Single precision floating point & 4 \\
D & Double precision floating point & 8 \\
C & Single precision complex & 8 \\
M & Double precision complex & 16 \\
P & Array Descriptor (32-bit) & 8 \\
Q & Array Descriptor (64-bit) & 16 \\
\hline
\end{tabular}

Notes. ${ }^{(\dagger)}$ Number of 8-bit bytes needed to contain all bits.

END keyword. This keyword has no associated value. Bytes 9 through 80 shall contain ASCII spaces (decimal 32 or hexadecimal 20).

\subsubsection{Other reserved keywords}

In addition to the reserved keywords defined in Sect. 4.4 .2 (except for EXTEND and BLOCKED), the following other reserved keywords may be used to describe the structure of a binary table data array. They are optional, but if they appear within a binary table extension header, they must be used as defined in this section of this standard.

TTYPEn keywords. The value field for this indexed keyword shall contain a character string giving the name of field $n$. It is strongly recommended that every field of the table be assigned a unique, case-insensitive name with this keyword, and it is recommended that the character string be composed only of upper and lower case letters, digits, and the underscore ("_,", decimal 95, hexadecimal 5F) character. Use of other characters is not recommended because it may be difficult to map the column names into variables in some languages (e.g., any hyphens, "*”, or "+" characters in the name may be confused with mathematical operators). String comparisons with the TTYPEn keyword values should not be case sensitive (e.g., "TIME" and "Time" should be interpreted as the same name).

TUNITn keywords. The value field shall contain a character string describing the physical units in which the quantity in field $n$, after any application of TSCALn and TZEROn, is expressed. Units must follow the prescriptions in Sect. 4.3.

TSCALn keywords. This indexed keyword shall be used, along with the TZEROn keyword, to linearly scale the values in the table field $n$ to transform them into the physical values that they represent using Eq. (7). It must not be used if the format of field $n$ is A, L, or X. For fields with all other data types, the value field shall contain a floating-point number representing the coefficient of the linear term in Eq. (7), which is used to compute the true physical value of the field, or, in the case of the complex data types $\mathrm{C}$ and $\mathrm{M}$, of the real part of the field, with the imaginary part of the scaling factor set to zero. The default value for this keyword is 1.0. For fields of type P or Q, the values of TSCALn and TZEROn are to be applied to the values in the data array in the heap area, not the values of the array descriptor (see Sect. 7.3.5).

TZEROn keywords. This indexed keyword shall be used, along with the TSCALn keyword, to linearly scale the values in the table field $n$ to transform them into the physical values that they represent using Eq. (7). It must not be used if the format of field $n$ is A, L, or X. For fields with all other data types, the value field shall contain a floating-point number representing the true physical value corresponding to a value of zero in field $n$ of the FITS file, or, in the case of the complex data types $C$ and $M$, in the real part of the field, with the imaginary part set to zero. The default value for this keyword is 0.0. Equation (7) is used to compute a true physical value from the quantity in field $n$. For fields of type P or Q, the values of TSCALn and TZEROn are to be applied to the values in the data array in the heap area, not the values of the array descriptor (see Sect. 7.3.5).

In addition to its use in representing floating-point values as scaled integers, the TZEROn keyword is also used when storing unsigned integer values in the field. In this special case the TSCALn keyword shall have the default value of 1.0 and the TZEROn keyword shall have one of the integer values shown in Table 19

Since the binary table format does not support a native unsigned integer data type (except for the unsigned 8-bit "B" column type), the unsigned values are stored in the field as native signed integers with the appropriate integer offset specified by the TZEROn keyword value shown in the table. For the byte column type, the converse technique can be used to store signed byte values as native unsigned values with the negative TZEROn offset. In each case, the physical value is computed by adding the offset specified by the TZEROn keyword to the native data type value that is stored in the table field.

TNULLn keywords. The value field for this indexed keyword shall contain the integer that represents an undefined value for field $n$ of data type $\mathrm{B}, \mathrm{I}, \mathrm{J}$ or $\mathrm{K}$, or $\mathrm{P}$ or $\mathrm{Q}$ array descriptor fields (Sect. 7.3.5) that point to B, I, J or K integer arrays. The keyword must not be used if field $n$ is of any other data type. The value of this keyword corresponds to the table column values before applying any transformation indicated by the TSCALn and TZEROn keywords.

If the TSCALn and TZEROn keywords do not have the default values of 1.0 and 0.0 , respectively, then the value of the TNULLn keyword must equal the actual value in the FITS file that is used to represent an undefined element and not the corresponding physical value (computed from Eq. (7)). To cite a specific, common example, unsigned 16-bit integers are represented in a signed integer column (with TFORMn = ' I') by setting TZEROn $=32768$ and TSCALn $=1$. If it is desired to use elements that have an unsigned value (i.e., the physical value) equal to 0 to represent undefined elements in the field, then the TNULLn keyword must be set to the value -32768 because that is the actual value stored in the FITS file for those elements in the field.

TDISPn keywords. The value field of this indexed keyword shall contain a character string describing the format recommended for displaying an ASCII text representation of the contents of field $n$. If the table value has been scaled, the physical value, derived using Eq. (7), shall be displayed. All elements in a field shall be displayed with a single, repeated format. For 
Table 19. Usage of TZEROn to represent non-default integer data types.

\begin{tabular}{rccrl}
\hline \hline TFORMn & $\begin{array}{c}\text { Native } \\
\text { data type }\end{array}$ & $\begin{array}{c}\text { Physical } \\
\text { data type }\end{array}$ & TZEROn & \\
\hline B & unsigned & signed byte & -128 & $\left(-2^{7}\right)$ \\
I & signed & unsigned 16-bit & 32768 & $\left(2^{15}\right)$ \\
J & signed & unsigned 32-bit & 2147483648 & $\left(2^{31}\right)$ \\
K & signed & unsigned 64-bit & 9223372036854775808 & $\left(2^{63}\right)$ \\
\hline
\end{tabular}

purposes of display, each byte of bit (type X) and byte (type B) arrays is treated as an unsigned integer. Arrays of type A may be terminated with a zero byte. Only the format codes in Table 20, interpreted as Fortran (ISO 2004) output formats, and discussed in more detail in Sect. 7.3.4, are permitted for encoding. The format codes must be specified in upper case. If the Bw.m, Ow.m, and $\mathrm{Zw}$. $\mathrm{m}$ formats are not readily available to the reader, the Iw . $\mathrm{m}$ display format may be used instead, and if the ENw.d and ESw.d formats are not available, Ew . d may be used. In the case of fields of type $\mathrm{P}$ or Q, the TDISPn value applies to the data array pointed to by the array descriptor (Sect. 7.3.5), not the values in the array descriptor itself.

THEAP keyword. The value field of this keyword shall contain an integer providing the separation, in bytes, between the start of the main data table and the start of a supplemental data area called the heap. The default value, which is also the minimum allowed value, shall be the product of the values of NAXIS1 and NAXIS2. This keyword shall not be used if the value of PCOUNT is zero. The use of this keyword is described in in Sect. 7.3.5.

TDIMn keywords. The value field of this indexed keyword shall contain a character string describing how to interpret the contents of field $n$ as a multi-dimensional array with a format of " $(l, m, n \ldots)$ " where $l, m, n, \ldots$ are the dimensions of the array. The data are ordered such that the array index of the first dimension given $(l)$ is the most rapidly varying and that of the last dimension given is the least rapidly varying. The total number of elements in the array equals the product of the dimensions specified in the TDIMn keyword. The size must be less than or equal to the repeat count on the TFORMn keyword, or, in the case of columns that have a "P" or "Q" TFORMn data type, less than or equal to the array length specified in the variable-length array descriptor (see Sect. 7.3.5). In the special case where the variablelength array descriptor has a size of zero, then the TDIMn keyword is not applicable. If the number of elements in the array implied by the TDIMn is less than the allocated size of the array in the FITS file, then the unused trailing elements should be interpreted as containing undefined fill values.

A character string is represented in a binary table by a onedimensional character array, as described under "Character" in the list of data types in Sect. 7.3.3. For example, a Fortran CHARACTER $* 20$ variable could be represented in a binary table as a character array declared as TFORMn $=$ '20A'. Arrays of strings, i.e., multi-dimensional character arrays, may be represented using the TDIMn notation. For example, if TFORMn = ' $60 \mathrm{~A}$ ' and TDIMn $=$ ' $(5,4,3)$ ', then the entry consists of a $4 \times 3$ array of strings of five characters each.

\subsubsection{Data sequence}

The data in a binary table extension shall consist of a main data table which may, but need not, be followed by additional bytes in the supplemental data area. The positions in the last data block after the last additional byte, or, if there are no additional bytes, the last character of the last row of the main data table, shall be filled by setting all bits to zero.

7.3.3.1. Main data table The table is constructed from a twodimensional byte array. The number of bytes in a row shall be specified by the value of the NAXIS1 keyword and the number of rows shall be specified by the NAXIS2 keyword of the associated header. Within a row, fields shall be stored in order of increasing column number, as determined from the $n$ of the TFORMn keywords. The number of bytes in a row and the number of rows in the table shall determine the size of the byte array. Every row in the array shall have the same number of bytes. The first row shall begin at the start of the data block immediately following the last header block. Subsequent rows shall begin immediately following the end of the previous row, with no intervening bytes, independent of the FITS block structure. Words need not be aligned along word boundaries.

Each row in the array shall consist of a sequence of from 0 to 999 fields as specified by the TFIELDS keyword. The number of elements in each field and their data type shall be specified by the TFORMn keyword in the associated header. A separate format keyword must be provided for each field. The location and format of fields shall be the same for every row. Fields may be empty, if the repeat count specified in the value of the TFORMn keyword of the header is 0 . Writers of binary tables should select a format appropriate to the form, range of values, and accuracy of the data in the table. The following data types, and no others, are permitted.

Logical. If the value of the TFORMn keyword specifies data type L, the contents of field $n$ shall consist of ASCII T indicating true or ASCII F, indicating false. A 0 byte (hexadecimal 00) indicates a NULL value.

Bit array. If the value of the TFORMn keyword specifies data type $\mathrm{X}$, the contents of field $n$ shall consist of a sequence of bits starting with the most significant bit; the bits following shall be in order of decreasing significance, ending with the least significant bit. A bit array shall be composed of an integral number of bytes, with those bits following the end of the data set to zero. No null value is defined for bit arrays.

Character. If the value of the TFORMn keyword specifies data type A, field $n$ shall contain a character string of zero or more members, composed of the restricted set of ASCII text characters. This character string may be terminated before the length specified by the repeat count by an ASCII NULL (hexadecimal code 00). Characters after the first ASCII NULL are not defined. A string with the number of characters specified by the repeat 
count is not NULL terminated. Null strings are defined by the presence of an ASCII NULL as the first character.

Unsigned 8-bit integer. If the value of the TFORMn keyword specifies data type $\mathrm{B}$, the data in field $n$ shall consist of unsigned 8-bit integers, with the most significant bit first, and subsequent bits in order of decreasing significance. Null values are given by the value of the associated TNULLn keyword. Signed integers can be represented using the convention described in Sect. 5.2.5.

16-Bit integer. If the value of the TFORMn keyword specifies data type I, the data in field $n$ shall consist of two's complement signed 16-bit integers, contained in two bytes. The most significant byte shall be first (big-endian byte order). Within each byte the most significant bit shall be first, and subsequent bits shall be in order of decreasing significance. Null values are given by the value of the associated TNULLn keyword. Unsigned integers can be represented using the convention described in Sect. 5.2.5.

32-Bit integer. If the value of the TFORMn keyword specifies data type J, the data in field $n$ shall consist of two's complement signed 32-bit integers, contained in four bytes. The most significant byte shall be first, and subsequent bytes shall be in order of decreasing significance (big-endian byte order). Within each byte, the most significant bit shall be first, and subsequent bits shall be in order of decreasing significance. Null values are given by the value of the associated TNULLn keyword. Unsigned integers can be represented using the convention described in Sect. 5.2.5.

64-Bit integer. If the value of the TFORMn keyword specifies data type $\mathrm{K}$, the data in field $n$ shall consist of two's complement signed 64-bit integers, contained in eight bytes. The most significant byte shall be first, and subsequent bytes shall be in order of decreasing significance. Within each byte, the most significant bit shall be first, and subsequent bits shall be in order of decreasing significance (big-endian byte order). Null values are given by the value of the associated TNULLn keyword. Unsigned integers can be represented using the convention described in Sect. 5.2.5.

Single precision floating point. If the value of the TFORMn keyword specifies data type E, the data in field $n$ shall consist of ANSI/IEEE-754 (IEEE 1985) 32-bit floating-point numbers, in big-endian byte order, as described in Appendix E. All IEEE special values are recognized. The IEEE NaN is used to represent null values.

Double precision floating point. If the value of the TFORMn keyword specifies data type D, the data in field $n$ shall consist of ANSI/IEEE-754 (IEEE 1985) 64-bit double precision floating-point numbers, in big-endian byte order, as described in Appendix E. All IEEE special values are recognized. The IEEE $\mathrm{NaN}$ is used to represent null values.

Single precision complex. If the value of the TFORMn keyword specifies data type $\mathrm{C}$, the data in field $n$ shall consist of a sequence of pairs of 32-bit single precision floating-point numbers. The first member of each pair shall represent the real part of a complex number, and the second member shall represent the imaginary part of that complex number. If either member contains an IEEE NaN, the entire complex value is null.

Double precision complex. If the value of the TFORMn keyword specifies data type $\mathrm{M}$, the data in field $n$ shall consist of a sequence of pairs of 64-bit double precision floating-point numbers. The first member of each pair shall represent the real part of a complex number, and the second member of the pair shall represent the imaginary part of that complex number. If either member contains an IEEE NaN, the entire complex value is null.

Array descriptor. The repeat count on the $\mathrm{P}$ and $\mathrm{Q}$ array descriptor fields must either have a value of 0 (denoting an empty field of zero bytes) or 1 . If the value of the TFORMn keyword specifies data type $1 \mathrm{P}$, the data in field $n$ shall consist of one pair of 32-bit integers. If the value of the TFORMn keyword specifies data type 1Q, the data in field $n$ shall consist of one pair of 64-bit integers. The meaning of these integers is defined in Sect. 7.3.5.

7.3.3.2. Bytes following main table. The main data table may be followed by a supplemental data area called the heap. The size of the supplemental data area, in bytes, is specified by the value of the PCOUNT keyword. The use of this data area is described in Sect. 7.3.5.

\subsubsection{Data display}

The indexed TDISPn keyword may be used to describe the recommended format for displaying an ASCII text representation of the contents of field $n$. The permitted display format codes for each type of data (i.e., character strings, logical, integer, or real) are given in Table 20 and described below.

Character data. If the table column contains a character string (with TFORMn = 'rA') then the TDISPn format code must be 'Aw' where $w$ is the number of characters to display. If the character datum has length less than or equal to $\mathrm{w}$, it is represented on output right-justified in a string of $w$ characters. If the character datum has length greater than $\mathrm{w}$, the first $\mathrm{w}$ characters of the datum are represented on output in a string of $w$ characters. Character data are not surrounded by single or double quotation marks unless those marks are themselves part of the data value.

Logical data. If the table column contains logical data (with TFORMn = ' $r L$ ') then the TDISPn format code must be 'Lw' where $\mathrm{w}$ is the width in characters of the display field. Logical data are represented on output with the character $\mathrm{T}$ for true or $\mathrm{F}$ for false right-justified in a space-filled string of $\mathrm{w}$ characters. A null value may be represented by a string of w space characters.

Integer data. If the table column contains integer data (with TFORMn = 'rX', 'rB', 'rI', ' $r J$ ', or ' $r K$ ') then the TDISPn format code may have any of these forms: Iw.m, Bw.m, $\mathrm{Ow}$.m, orZw.m. The default value of $m$ is one and the ". $m$ " is $o p$ tional. The first letter of the code specifies the number base for the encoding with I for decimal (10), B for binary (2), 0 for octal (8), and Z for hexadecimal (16). Hexadecimal format uses the upper-case letters $\mathrm{A}$ through $\mathrm{F}$ to represent decimal values 10 through 15 . The output field consists of $\mathrm{w}$ characters containing zero or more leading spaces followed by a minus sign if the 
Table 20. Valid TDISPn format values in BINTABLE extensions.

\begin{aligned} & \hline \hline Field value Data type \\ & \hline Aw Character \\ & Lw Logical \\ & Iw.m Integer \\ & Bw.m Binary, integers only \\ & Ow.m Octal, integers only \\ & Zw.m Hexadecimal, integers only \\ & Fw.d Floating-point, fixed decimal notation \\ & Ew.dEe Floating-point, exponential notation \\ & ENw.d Engineering; E format with exponent multiple of three \\ & ESw.d Scientific; same as EN but non-zero leading digit if not zero \\ & Gw.dEe General; appears as F if significance not lost, else E. \\ & Dw.dEe Floating-point, exponential notation \\ & \hline\end{aligned}

Notes. $w$ is the width in characters of displayed values, $m$ is the minimum number of digits displayed, $d$ is the number of digits to right of decimal, and e is number of digits in exponent. The .m and Ee fields are optional.

internal datum is negative (only in the case of decimal encoding with the I format code) followed by the magnitude of the internal datum in the form of an unsigned integer constant in the specified number base with only as many leading zeros as are needed to have at least $m$ numeric digits. Note that $m \leq w$ is allowed if all values are positive, but $m<w$ is required if any values are negative. If the number of digits required to represent the integer datum exceeds $w$, then the output field consists of a string of $\mathrm{w}$ asterisk $(*)$ characters.

Real data. If the table column contains real data (with TFORMn $=$ ' $r E$ ', or ' $r D$ ') or contains integer data (with any of the TFORMn format codes listed in the previous paragraph) which are recommended to be displayed as real values (i.e., especially in cases where the integer values represent scaled physical values using Eq. (7)), then the TDISPn format code may have any of these forms: Fw.d, Ew.dEe, Dw.dEe, ENw.d, or ESw.d. In all cases, the output is a string of $\mathrm{w}$ characters including the decimal point, any sign characters, and any exponent including the exponent's indicators, signs, and values. If the number of digits required to represent the real datum exceeds $\mathrm{w}$, then the output field consists of a string of $\mathrm{w}$ asterisk (*) characters. In all cases, $\mathrm{d}$ specifies the number of digits to appear to the right of the decimal point.

The $\mathrm{F}$ format code output field consists of $\mathrm{w}-\mathrm{d}-1$ characters containing zero or more leading spaces followed by a minus sign if the internal datum is negative followed by the absolute magnitude of the internal datum in the form of an unsigned integer constant. These characters are followed by a decimal point (“.") and d characters giving the fractional part of the internal datum, rounded by the normal rules of arithmetic to $\mathrm{d}$ fractional digits.

For the $\mathrm{E}$ and $\mathrm{D}$ format codes, an exponent is taken such that the fraction $0.1 \leq \mid$ datum $\mid / 10^{\text {exponent }}<1.0$. The fraction (with appropriate sign) is output with an $\mathrm{F}$ format of width $\mathrm{w}-\mathrm{e}-2$ characters with $d$ characters after the decimal followed by an $E$ or $\mathrm{D}$ followed by the exponent as a signed $\mathrm{e}+1$ character integer with leading zeros as needed. The default value of e is 2 when the Ee portion of the format code is omitted. If the exponent value will not fit in $\mathrm{e}+1$ characters but will fit in $\mathrm{e}+2$ then the $\mathrm{E}$ (or D) is omitted and the wider field used. If the exponent value will not fit (with a sign character) in e +2 characters, then the entire w-character output field is filled with asterisks (*).

The ES format code is processed in the same manner as the E format code except that the exponent is taken so that $1.0 \leq$ fraction $<10$.
The EN format code is processed in the same manner as the $E$ format code except that the exponent is taken to be an integer multiple of three and so that $1.0 \leq$ fraction $<1000.0$. All real format codes have number base 10 . There is no difference between $\mathrm{E}$ and $\mathrm{D}$ format codes on input other than an implication with the latter of greater precision in the internal datum.

The Gw . dEe format code may be used with data of any type. For data of type integer, logical, or character, it is equivalent to Iw, Lw, or Aw, respectively. For data of type real, it is equivalent to an $\mathrm{F}$ format (with different numbers of characters after the decimal) when that format will accurately represent the value and is equivalent to an $\mathrm{E}$ format when the number (in absolute value) is either very small or very large. Specifically, for real values outside the range $0.1-0.5 \times 10^{-\mathrm{d}-1} \leq$ value $<10^{\mathrm{d}}-0.5$, it is equivalent to $\mathrm{Ew} . \mathrm{dEe}$. For real values within the above range, it is equivalent to $\mathrm{Fw}^{\prime} \cdot \mathrm{d}^{\prime}$ followed by $2+\mathrm{e}$ spaces, where $\mathrm{w}^{\prime}=\mathrm{w}-\mathrm{e}-2$ and $\mathrm{d}^{\prime}=\mathrm{d}-k$ for $k=0,1, \ldots, \mathrm{d}$ if the real datum value lies in the range $10^{k-1}\left(1-0.5 \times 10^{-\mathrm{d}}\right) \leq$ value $\leq$ $10^{k}\left(1-0.5 \times 10^{-\mathrm{d}}\right)$.

Complex data. If the table column contains complex data (with TFORMn = ' $r C$ ', or ' $r M$ ') then the may be displayed with any of the real data formats as described above. The same format is used for the real and imaginary parts. It is recommended that the two values be separated by a comma and enclosed in parentheses with a total field width of $2 w+3$.

\subsubsection{Variable-length arrays}

One of the most attractive features of binary tables is that any field of the table can be an array. In the standard case this is a fixed-size array, i.e., a fixed amount of storage is allocated in each row for the array data - whether it is used or not. This is fine so long as the arrays are small or a fixed amount of array data will be stored in each field, but if the stored array length varies for different rows, it is necessary to impose a fixed upper limit on the size of the array that can be stored. If this upper limit is made too large excessive wasted space can result and the binary table mechanism becomes seriously inefficient. If the limit is set too low then storing certain types of data in the table could become impossible.

The variable-length array construct presented here was devised to deal with this problem. Variable-length arrays are implemented in such a way that, even if a table contains such arrays, 
a simple reader program that does not understand variable-length arrays will still be able to read the main data table (in other words a table containing variable-length arrays conforms to the basic binary table standard). The implementation chosen is such that the rows in the main data table remain fixed in size even if the table contains a variable-length array field, allowing efficient random access to the main data table.

Variable-length arrays are logically equivalent to regular static arrays, the only differences being 1) the length of the stored array can differ for different rows; and 2) the array data are not stored directly in the main data table. Since a field of any data type can be a static array, a field of any data type can also be a variable-length array (excluding the type $P$ and $Q$ variable-length array descriptors themselves, which are not a data type so much as a storage-class specifier). Other established FITS conventions that apply to static arrays will generally apply as well to variablelength arrays.

A variable-length array is declared in the table header with one of the following two special field data type specifiers

$\operatorname{rPt}\left(\mathrm{e}_{\max }\right)$

$\operatorname{rQt}\left(\mathrm{e}_{\max }\right)$

where the "P" or " $Q$ " indicates the presence of an array descriptor (described below), the element count $\mathrm{r}$ should be 0,1 , or absent, $\mathrm{t}$ is a character denoting the data type of the array data $(\mathrm{L}, \mathrm{X}, \mathrm{B}$, $\mathrm{I}, \mathrm{J}, \mathrm{K}$, etc., but not $\mathrm{P}$ or $\mathrm{Q}$ ), and $\mathrm{e}_{\max }$ is a quantity guaranteed to be equal to or greater than the maximum number of elements of type $t$ actually stored in any row of the table. There is no built-in upper limit on the size of a stored array (other than the fundamental limit imposed by the range of the array descriptor, defined below); $\mathrm{e}_{\max }$ merely reflects the size of the largest array actually stored in the table, and is provided to avoid the need to preview the table when, for example, reading a table containing variable-length elements into a database that supports only fixedsize arrays. There may be additional characters in the TFORMn keyword following the $\mathrm{e}_{\max }$.

For example,

TFORM8 = 'PB(1800)' / Variable byte array

indicates that field 8 of the table is a variable-length array of type byte, with a maximum stored array length not to exceed 1800 array elements (bytes in this case).

The data for the variable-length arrays in a table are not stored in the main data table; they are stored in a supplemental data area, the heap, following the main data table. What is stored in the main data table field is an array descriptor. This consists of two 32-bit signed integer values in the case of "P" array descriptors, or two 64-bit signed integer values in the case of "Q" array descriptors: the number of elements (array length) of the stored array, followed by the zero-indexed byte offset of the first element of the array, measured from the start of the heap area. The meaning of a negative value for either of these integers is not defined by this standard. Storage for the array is contiguous. The array descriptor for field $N$ as it would appear embedded in a table row is illustrated symbolically below:

... [field $N-1][($ nelem, offset)] [field $N+1] \ldots$

If the stored array length is zero there is no array data, and the offset value is undefined (it should be set to zero). The storage referenced by an array descriptor must lie entirely within the heap area; negative offsets are not permitted.
A binary table containing variable-length arrays consists of three principal segments, as follows:

[table header] [main data table] (optional gap) [heap area].

The table header consists of one or more 2880-byte header blocks with the last block indicated by the keyword END somewhere in the block. The main data table begins with the first data block following the last header block and is NAXIS1 1 NAXIS2 bytes in length. The zero indexed byte offset to the start of the heap, measured from the start of the main data table, may be given by the THEAP keyword in the header. If this keyword is missing then the heap begins with the byte immediately following main data table (i.e., the default value of THEAP is NAXIS $1 \times$ NAXIS2). This default value is the minimum allowed value for the THEAP keyword, because any smaller value would imply that the heap and the main data table overlap. If the THEAP keyword has a value larger than this default value, then there is a gap between the end of the main data table and the start of the heap. The total length in bytes of the supplemental data area following the main data table (gap plus heap) is given by the PCOUNT keyword in the table header.

For example, suppose a table contains five rows which are each 168 bytes long, with a heap area 3000 bytes long, beginning at an offset of 2880, thereby aligning the main data table and heap areas on data block boundaries (this alignment is not necessarily recommended but is useful for this example). The data portion of the table consists of three 2880-byte data blocks: the first block contains the 840 bytes from the five rows of the main data table followed by 2040 fill bytes; the heap completely fills the second block; the third block contains the remaining 120 bytes of the heap followed by 2760 fill bytes. PCOUNT gives the total number of bytes from the end of the main data table to the end of the heap and in this example has a value of $2040+2880+120=5040$. This is expressed in the table header as:

$\begin{aligned} \text { NAXIS1 } & 168 / \text { Width of table row in bytes } \\ \text { NAXIS2 } & 5 / \text { Number of rows in table } \\ \text { PCOUNT } & =5040 / \text { Random parameter count } \\ \ldots & \\ \text { THEAP } & =2880 / \text { Byte offset of heap area. }\end{aligned}$

The values of TSCALn and TZEROn for variable-length array column entries are to be applied to the values in the data array in the heap area, not the values of the array descriptor. These keywords can be used to scale data values in either static or variable-length arrays.

\subsubsection{Variable-length-array guidelines}

While the above description is sufficient to define the required features of the variable-length array implementation, some hints regarding usage of the variable-length array facility might also be useful.

Programs that read binary tables should take care to not assume more about the physical layout of the table than is required by the specification. For example, there are no requirements on the alignment of data within the heap. If efficient runtime access is a concern one might want to design the table so that data arrays are aligned to the size of an array element. In another case one might want to minimize storage and forgo any efforts at alignment (by careful design it is often possible to achieve both goals). Variable-length array data may be stored in the heap in any order, i.e., the data for row $N+1$ are not necessarily stored 
at a larger offset than that for row $N$. There may be gaps in the heap where no data are stored. Pointer aliasing is permitted, i.e., the array descriptors for two or more arrays may point to the same storage location (this could be used to save storage if two or more arrays are identical).

Byte arrays are a special case because they can be used to store a "typeless" data sequence. Since FITS is a machineindependent storage format, some form of machine-specific data conversion (byte swapping, floating-point format conversion) is implied when accessing stored data with types such as integer and floating, but byte arrays are copied to and from external storage without any form of conversion.

An important feature of variable-length arrays is that it is possible that the stored array length may be zero. This makes it possible to have a column of the table for which, typically, no data are present in each stored row. When data are present the stored array can be as large as necessary. This can be useful when storing complex objects as rows in a table.

Accessing a binary table stored on a random access storage medium is straightforward. Since the rows of data in the main data table are fixed in size they can be randomly accessed given the row number, by computing the offset. Once the row has been read in, any variable-length array data can be directly accessed using the element count and offset given by the array descriptor stored in that row.

Reading a binary table stored on a sequential access storage medium requires that a table of array descriptors be built up as the main data table rows are read in. Once all the table rows have been read, the array descriptors are sorted by the offset of the array data in the heap. As the heap data are read, arrays are extracted sequentially from the heap and stored in the affected rows using the back pointers to the row and field from the table of array descriptors. Since array aliasing is permitted, it might be necessary to store a given array in more than one field or row.

Variable-length arrays are more complicated than regular static arrays and might not be supported by some software systems. The producers of FITS data products should consider the capabilities of the likely recipients of their files when deciding whether or not to use this format, and as a general rule should use it only in cases where it provides significant advantages over the simpler fixed-length array format. In particular, the use of variable-length arrays might present difficulties for applications that ingest the FITS file via a sequential input stream because the application cannot fully process any rows in the table until after the entire fixed-length table and potentially the entire heap has been transmitted as outlined in the previous paragraph.

\section{World coordinate systems}

Representations of the mapping between image coordinates and physical (i.e., world) coordinate systems (WCSs) may be represented within FITS HDUs. The keywords that are used to express these mappings are now rigorously defined in a series of papers on world coordinate systems (Greisen \& Calabretta 2002), celestial coordinate systems (Calabretta \& Greisen 2002), and spectral coordinate systems (Greisen et al. 2006). An additional spherical projection, called HEALPix, is defined in reference (Calabretta \& Roukema 2007). These WCS papers have been formally approved by the IAUFWG and therefore are incorporated by reference as an official part of this Standard. The reader should refer to these papers for additional details and background information that cannot be included here. Various updates and corrections to the primary WCS papers have been compiled by the authors, and are reflected in this section.
Therefore, where conflicts exist, the description in this Standard will prevail.

\subsection{Basic concepts}

Rather than store world coordinates separately for each datum, the regular lattice structure of a FITS image offers the possibility of defining rules for computing world coordinates at each point. As stated in Sect. 3.3.2 and depicted in Fig. 1, image array data are addressed via integral array indices that range in value from 1 to NAXIS $j$ on axis $j$. Recognizing that image data values may have an extent, for example an angular separation, spectral channel width or time span, and thus that it may make sense to interpolate between them, these integral array indices may be generalized to floating-point pixel coordinates. Integral pixel coordinate values coincide with the corresponding array indices, while fractional pixel coordinate values lie between array indices and thus imply interpolation. Pixel coordinate values are defined at all points within the image lattice and outside it (except along conventional axes, see Sect. 8.5). They form the basis of the world coordinate formalism in FITS depicted schematically in Fig. 2.

The essence of representing world coordinate systems in FITS is the association of various reserved keywords with elements of a transformation (or a series of transformations), or with parameters of a projection function. The conversion from pixel coordinates in the data array to world coordinates is simply a matter of applying the specified transformations (in order) via the appropriate keyword values; conversely, defining a WCS for an image amounts to solving for the elements of the transformation matrix(es) or coefficients of the function(s) of interest and recording them in the form of WCS keyword values. The description of the WCS systems and their expression in FITS HDUs is quite extensive and detailed, but is aided by a careful choice of notation. Key elements of the notation are summarized in Table 21, and are used throughout this section. The formal definitions of the keywords appear in the following subsections.

The conversion of image pixel coordinates to world coordinates is a multi-step process, as illustrated in Fig. 2.

For all coordinate types, the first step is a linear transformation applied via matrix multiplication of the vector of pixel coordinate elements, $p_{j}$ :

$q_{i}=\sum_{j=1}^{N} m_{i j}\left(p_{j}-r_{j}\right)$

where $r_{j}$ are the pixel coordinate elements of the reference point, $j$ indexes the pixel axis, and $i$ the world axis. The $m_{i j}$ matrix is a non-singular, square matrix of dimension $N \times N$, where $N$ is the number of world coordinate axes. The elements $q_{i}$ of the resulting intermediate pixel coordinate vector are offsets, in dimensionless pixel units, from the reference point along axes coincident with those of the intermediate world coordinates. Thus, the conversion of $q_{i}$ to the corresponding intermediate world coordinate element $x_{i}$ is a simple scale:

$x_{i}=s_{i} q_{i}$.

There are three conventions for associating FITS keywords with the above transformations. In the first formalism, the matrix elements $m_{i j}$ are encoded in the PCi_j keywords and the scale factors $s_{i}$ are encoded in the CDELT $i$ keywords, which must have 
Table 21. WCS and celestial coordinates notation.

\begin{tabular}{lll}
\hline \hline Variable(s) & Meaning & Related FITS keywords \\
\hline$i$ & Index variable for world coordinates & \\
$j$ & Index variable for pixel coordinates & \\
$a$ & Alternative WCS version code & \\
$p_{j}$ & Pixel coordinates & CRPIXja \\
$r_{j}$ & Reference pixel coordinates & CDi_ja or PCi_ja \\
$m_{i j}$ & Linear transformation matrix & CDELTia \\
$s_{i}$ & Coordinate scales & \\
$(x, y)$ & Projection plane coordinates & \\
$(\phi, \theta)$ & Native longitude and latitude & \\
$(\alpha, \delta)$ & Celestial longitude and latitude & PVi_1 $a^{\dagger}$, PVi_2a $a^{\dagger}$ \\
$\left(\phi_{0}, \theta_{0}\right)$ & Native longitude and latitude of the fiducial point & \\
$\left(\alpha_{0}, \delta_{0}\right)$ & Celestial longitude and latitude of the fiducial point & CRVALia \\
$\left(\alpha_{\mathrm{p}}, \delta_{\mathrm{p}}\right)$ & Celestial longitude and latitude of the native pole & \\
$\left(\phi_{\mathrm{p}}, \theta_{\mathrm{p}}\right)$ & Native longitude and latitude of the celestial pole & LONPOLE $a$ (=PVi_3a $\left.a^{\dagger}\right)$, \\
& & LATPOLE $a\left(=\mathrm{PV} i_{-} 4 a^{\dagger}\right)$ \\
\hline
\end{tabular}

Notes. ${ }^{(\dagger)}$ Associated with longitude axis $i$.

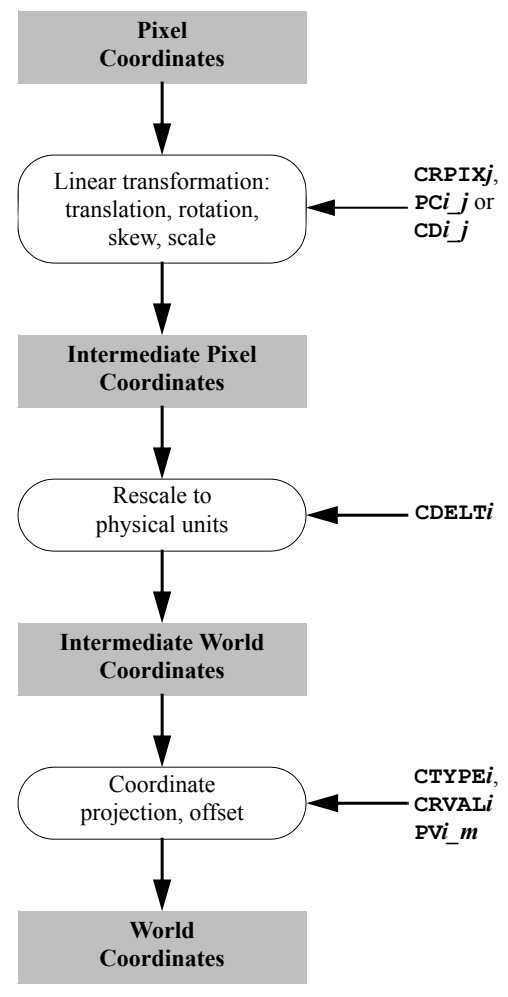

Fig. 2. A schematic view of converting pixel coordinates to world coordinates.

non-zero values. In the second formalism Eqs. (9) and (10) are combined as

$x_{i}=\sum_{j=1}^{N}\left(s_{i} m_{i j}\right)\left(p_{j}-r_{j}\right)$

and the $\mathrm{CD} i_{-} j$ keywords encode the product $s_{i} m_{i j}$. The third convention was widely used before the development of the two previously described conventions and uses the CDELT $i$ keywords to define the image scale and the CROTA2 keyword to define a bulk rotation of the image plane. Use of the CROTA2 keyword is now deprecated, and instead the newer $\mathrm{PC} i_{-} j$ or $\mathrm{CD} i_{-} j$ keywords are recommended because they allow for skewed axes and fully general rotation of multi-dimensional arrays. The CDELT $i$ and
CROTA2 keywords may co-exist with the CDi_j keywords (but the CROTA2 must not occur with the PCi_j keywords) as an aid to old FITS interpreters, but these keywords must be ignored by software that supports the $\mathrm{CD} i \_j$ keyword convention. In all these formalisms the reference pixel coordinates $r_{j}$ are encoded in the CRPIX $i$ keywords, and the world coordinates at the reference point are encoded in the CRVAL $i$ keywords. For additional details, see Greisen \& Calabretta (2002).

The third step of the process, computing the final world coordinates, depends on the type of coordinate system, which is indicated with the value of the CTYPE $i$ keyword. For some simple, linear cases an appropriate choice of normalization for the scale factors allows the world coordinates to be taken directly (or by applying a constant offset) from the $x_{i}$ (e.g., some spectra). In other cases it is more complicated, and may require the application of some non-linear algorithm (e.g., a projection, as for celestial coordinates), which may require the specification of additional parameters. Where necessary, numeric parameter values for non-linear algorithms must be specified via PV $i \_m$ keywords and character-valued parameters will be specified via PSi_ $m$ keywords, where $m$ is the parameter number.

The application of these formalisms to coordinate systems of interest is discussed in the following sub-sections: Sect. 8.2 describes general WCS representations (see Greisen \& Calabretta 2002), Sect. 8.3 describes celestial coordinate systems (see Calabretta \& Greisen 2002), and Sect. 8.4 describes spectral coordinate systems (see Greisen et al. 2006).

\subsection{World coordinate system representations}

A variety of keywords have been reserved for computing the coordinate values that are to be associated with any pixel location within an array. The full set is given in Table 22; those in most common usage are defined in detail below for convenience. Coordinate system specifications may appear in HDUs that contain simple images in the primary array or in an image extension. Images may also be stored in a multi-dimensional vector cell of a binary table, or as a tabulated list of pixel locations (and optionally, the pixel value) in a table. In these last two types of image representations, the WCS keywords have a different naming convention which reflects the needs of the tabular data structure and the 8-character limit for keyword lengths, but otherwise follow exactly the same rules for type, usage, and default values. 
Table 22. Reserved WCS keywords.

\begin{tabular}{|c|c|c|c|}
\hline \multirow{2}{*}{$\begin{array}{l}\text { Keyword } \\
\text { Description }\end{array}$} & \multirow{2}{*}{$\begin{array}{l}\text { Primary } \\
\text { Array }\end{array}$} & BINTABLE vector & Pixel List \\
\hline & & alternative & alternative \\
\hline Coordinate dimensionality & WCSAXES $a$ & WCAXna & \\
\hline Axis type & CTYPEia & $i$ CTYPn & TCTYPn \\
\hline Axis units & CUNITia & $i$ CUNIn & TCUNIn \\
\hline Reference value & CRVALia & $i \mathrm{CRVL} n$ & TCRVLn \\
\hline Coordinate increment & CDELTia & $i \mathrm{CDLT} n$ & TCDLTn \\
\hline Reference point & CRPIXja & jCRPXn & TCRPX $n$ \\
\hline Coordinate rotation ${ }^{1}$ & CROTA $i$ & $i \mathrm{CROT} n$ & TCROT $n$ \\
\hline Transformation matrix ${ }^{2}$ & $\mathrm{PC} i \_j a$ & ijPCna & $\mathrm{TPC} n \_k a$ or TP $n \_k a$ \\
\hline Transformation matrix ${ }^{2}$ & $\mathrm{CD} i$ ja & ijCDna & $\mathrm{TCD} n \_k a$ or TCn_ka \\
\hline Coordinate parameter & PVi_ma & $i \mathrm{PV} n \_m a$ or $i \mathrm{~V} n \_m a$ & $\mathrm{TPV} n \_m a$ or TVn_ma \\
\hline Coordinate parameter array & . & $i \mathrm{~V} n \_\mathrm{X} a$ & $\ldots$ \\
\hline Coordinate parameter & PSi_ma & $i \mathrm{PS} n \_m a$ or $i \mathrm{~S} n \_m a$ & TPSn_ma or TSn_ma \\
\hline Coordinate name & WCSNAME $a$ & WCSNna & WCSna or TWCSna \\
\hline Coordinate axis name & CNAME $i a$ & $i$ CNAna & TCNAna \\
\hline Random error & CRDERia & iCRDna & TCRDna \\
\hline Systematic error & CSYERia & iCSYna & TCSYna \\
\hline WCS cross-reference target & $\ldots$ & WCSTna & $\ldots$ \\
\hline WCS cross reference & $\ldots$ & WCSXna & $\ldots$ \\
\hline Coordinate rotation & LONPOLE $a$ & LONPna & LONPna \\
\hline Coordinate rotation & LATPOLE $a$ & LATPna & LATPna \\
\hline Coordinate epoch & EQUINOX $a$ & EQUIna & EQUIna \\
\hline Coordinate epoch ${ }^{3}$ & EPOCH & EPOCH & EPOCH \\
\hline Date of observation & MJD-OBS & $\mathrm{MJDOB} n$ & $\mathrm{MJDOB} n$ \\
\hline Average date of observation & MJD-AVG & MJDA $n$ & MJDA $n$ \\
\hline Date/time of observation & DATE-OBS & DOBSn & DOBSn \\
\hline Average date/time of obs & DATE-AVG & DAVGn & DAVGn \\
\hline Reference frame & $\begin{array}{l}\text { RADESYS } a \text { or } \\
\text { RADECSYS }^{4}\end{array}$ & RADEna & RADE $n a$ \\
\hline Line rest frequency $(\mathrm{Hz})$ & $\begin{array}{l}\text { RESTFRQ } a \text { or } \\
\text { RESTFREQ }^{4}\end{array}$ & $\begin{array}{c}\text { RFRQna } \\
\ldots\end{array}$ & $\begin{array}{c}\text { RFRQna } \\
\ldots\end{array}$ \\
\hline Line rest vacuum wavelength (m) & RESTWAV $a$ & RWAVna & RWAVna \\
\hline Spectral reference frame & SPECSYS $a$ & SPECna & SPECna \\
\hline Spectral reference frame & SSYSOBS $a$ & SOBSna & SOBSna \\
\hline Spectral reference frame & SSYSSRC $a$ & SSRCna & SSRCna \\
\hline Observation $X(\mathrm{~m})$ & OBSGEO-X & OBSGX $n$ & OBSGX $n$ \\
\hline Observation $Y(\mathrm{~m})$ & OBSGEO-Y & OBSGY $n$ & OBSGY $n$ \\
\hline Observation $Z$ (m) & OBSGEO-Z & OBSGZn & OBSGZn \\
\hline Radial velocity $\left(\mathrm{m} \mathrm{s}^{-1}\right)$ & VELOSYS $a$ & VSYSna & VSYSna \\
\hline Redshift of source & ZSOURCE $a$ & ZSOUna & ZSOUna \\
\hline Angle of true velocity & VELANGL $a$ & VANGna & VANGna \\
\hline
\end{tabular}

Notes. The indexes $j$ and $i$ are pixel and intermediate world coordinate axis numbers, respectively. Within a table, the index $n$ refers to a column number, and $m$ refers to a coordinate parameter number. The index $k$ also refers to a column number. The indicator $a$ is either blank (for the primary coordinate description) or a character A through $\mathrm{Z}$ that specifies the coordinate version. See text.

(1) CROTA $i$ form is deprecated but still in use. It must not be used with PC $i_{-} j, \mathrm{PV} i \_m$, and PS $i_{-} m .{ }^{(2)} \mathrm{PC} i_{-} j$ and $\mathrm{CD} i \_j$ forms of the transformation matrix are mutually exclusive, and must not appear together in the same HDU. ${ }^{(3)}$ EPOCH is deprecated. Use EQUINOX instead. ${ }^{(4)}$ These 8-character keywords are deprecated; the 7-character forms, which can include an alternate version code letter at the end, should be used instead.

See reference Calabretta \& Greisen (2002) for example usage of these keywords. All forms of these reserved keywords must be used only as specified in this Standard.

The keywords given below constitute a complete set of fundamental attributes for a WCS description. Although their inclusion in an HDU is optional, FITS writers should include a complete set of keywords when describing a WCS. In the event that some keywords are missing, default values must be assumed, as specified below.

WCSAXES - [integer; default: NAXIS, or larger of WCS indexes $i$ or $j$ ]. Number of axes in the WCS description. This keyword, if present, must precede all WCS keywords except NAXIS in the HDU. The value of WCSAXES may exceed the number of pixel axes for the HDU.
CTYPE $i$ - [character; indexed; default: “" (i.e. a linear, undefined axis)]. Type for the intermediate coordinate axis $i$. Any coordinate type that is not covered by this standard or an officially recognized FITS convention shall be taken to be linear. All non-linear coordinate system names must be expressed in "4-3" form: the first four characters specify the coordinate type, the fifth character is a hyphen ("-"), and the remaining three characters specify an algorithm code for computing the world coordinate value. Coordinate types with names of less than four characters are padded on the right with hyphens, and algorithm codes with less than three characters are padded on the right with blanks ${ }^{10}$. Algorithm codes should be three characters.

10 Example: "RA---UV". 
CUNIT $i$ - [character; indexed; default: “" (i.e., undefined)]. Physical units of CRVAL and CDELT for axis $i$. Note that units should always be specified (see Sect. 4.3). Units for celestial coordinate systems defined in this Standard must be degrees. CRPIXj - [floating point; indexed; default: 0.0]. Location of the reference point in the image for axis $j$ corresponding to $r_{j}$ in Eq. (9). Note that the reference point may lie outside the image and that the first pixel in the image has pixel coordinates $(1.0,1.0, \ldots)$.

CRVAL $i$ - [floating point; indexed; default: 0.0]. World Coordinate value at the reference point of axis $i$.

CDELT $i$ - [floating point; indexed; default: 1.0]. Increment of the world coordinate at the reference point for axis $i$. The value must not be zero.

CROTA $i$ - [floating point; indexed; default: 0.0]. The amount of rotation from the standard coordinate system to a different coordinate system. Further use of this of this keyword is deprecated, in favor of the newer formalisms that use the CD $i_{-} j$ or $\mathrm{PC} i_{-} j$ keywords to define the rotation.

PC $i \_j-$ [floating point; defaults: 1.0 when $i=j, 0.0$ otherwise]. Linear transformation matrix between pixel axes $j$ and intermediate coordinate axes $i$. The PC $i_{-} j$ matrix must not be singular.

$\mathrm{CD} i_{-} j-$ [floating point; defaults: 0.0 , but see below]. Linear transformation matrix (with scale) between pixel axes $j$ and intermediate coordinate axes $i$. This nomenclature is equivalent to $\mathrm{PC} i i_{-} j$ when CDELT $i$ is unity. The $\mathrm{CD} i j$ matrix must $n o t$ be singular. Note that the $\mathrm{CD} i{ }_{-} j$ formalism is an exclusive alternative to $\mathrm{PC} i_{-} j$, and the $\mathrm{CD} i_{-} j$ and $\mathrm{PC} i_{-} j$ keywords must not appear together within an HDU.

In addition to the restrictions noted above, if any $\mathrm{CD} i j j$ keywords are present in the HDU, all other unspecified $\mathrm{CD} i \_j$ keywords shall default to zero. If no $\mathrm{CD} i i_{-} j$ keywords are present then the header shall be interpreted as being in $\mathrm{PC} i_{-} j$ form whether or not any $\mathrm{PC} i j j$ keywords are actually present in the HDU.

Some non-linear algorithms that describe the transformation between pixel and intermediate coordinate axes require parameter values. A few non-linear algorithms also require charactervalued parameters, e.g., table lookups require the names of the table extension and the columns to be used. Where necessary parameter values must be specified via the following keywords:

PVi_m - [floating point]. Numeric parameter values for intermediate world coordinate axis $i$, where $m$ is the parameter number. Leading zeros must not be used, and $m$ may have only values in the range 0 through 99 , and that are defined for the particular non-linear algorithm.

PSi_m - [character]. Character-valued parameters for intermediate world coordinate axis $i$, where $m$ is the parameter number. Leading zeros must not be used, and $m$ may have only values in the range 0 through 99, and that are defined for the particular non-linear algorithm.

The following keywords, while not essential for a complete specification of an image WCS, can be extremely useful for readers to interpret the accuracy of the WCS representation of the image.

CRDER $i$ - [floating point; default: 0.0]. Random error in coordinate $i$, which must be non-negative.

CSYER $i$ - [floating point; default: 0.0]. Systematic error in coordinate $i$, which must be non-negative.

These values should give a representative average value of the error over the range of the coordinate in the HDU. The total error in the coordinates would be given by summing the individual errors in quadrature.

\subsubsection{Alternative WCS axis descriptions}

In some cases it is useful to describe an image with more than one coordinate type ${ }^{11}$. Alternative WCS descriptions may be added to the header by adding the appropriate sets of WCS keywords, and appending to all keywords in each set an alphabetic code in the range A through $\mathrm{Z}$. Keywords that may be used in this way to specify a coordinate system version are indicated in Table 22 with the suffix $a$. All implied keywords with this encoding are reserved keywords, and must only be used in FITS HDUs as specified in this Standard. The axis numbers must lie in the range 1 through 99, and the coordinate parameter $m$ must lie in the range 0 through 99 , both with no leading zeros.

The primary version of the WCS description is that specified with $a$ as the blank character ${ }^{12}$. Alternative axis descriptions are optional, but must not be specified unless the primary WCS description is also specified. If an alternative WCS description is specified, all coordinate keywords for that version must be given even if the values do not differ from those of the primary version. Rules for the default values of alternative coordinate descriptions are the same as those for the primary description. The alternative descriptions are computed in the same fashion as the primary coordinates. The type of coordinate depends on the value of CTYPEia, and may be linear in one of the alternative descriptions and non-linear in another.

The alternative version codes are selected by the FITS writer; there is no requirement that the codes be used in alphabetic sequence, nor that one coordinate version differ in its parameter values from another. An optional keyword WCSNAME $a$ is also defined to name, and otherwise document, the various versions of WCS descriptions:

WCSNAME $a$ - [character; default for $a$ : " " (i.e., blank, for the primary WCS, else a character A through Z that specifies the coordinate version]. Name of the world coordinate system represented by the WCS keywords with the suffix $a$. Its primary function is to provide a means by which to specify a particular WCS if multiple versions are defined in the HDU.

\subsection{Celestial coordinate system representations}

The conversion from intermediate world coordinates $(x, y)$ in the plane of projection to celestial coordinates involves two steps: a spherical projection to native longitude and latitude $(\phi, \theta)$, defined in terms of a convenient coordinate system (i.e., $n a$ tive spherical coordinates), followed by a spherical rotation of these native coordinates to the required celestial coordinate system $(\alpha, \delta)$. The algorithm to be used to define the spherical projection must be encoded in the CTYPE $i$ keyword as the threeletter algorithm code, the allowed values for which are specified in Table 23 and defined in references Calabretta \& Greisen (2002) and Calabretta \& Roukema (2007). The target celestial coordinate system is also encoded into the left-most portion of the CTYPE $i$ keyword as the coordinate type.

For the final step, the parameter LONPOLE $a$ must be specified, which is the native longitude of the celestial pole, $\phi_{\mathrm{p}}$. For certain projections (such as cylindricals and conics, which

11 Examples include the frequency, velocity, and wavelength along a spectral axis (only one of which, of course, could be linear), or the position along an imaging detector in both meters and degrees on the sky. 12 There are a number of keywords (e.g. ijPCna) where the $a$ could be pushed off the 8-char keyword name for plausible values of $i, j, k, n$, and $m$. In such cases $a$ is still said to be "blank" although it is not the blank character. 
Table 23. Reserved celestial coordinate algorithm codes.

\begin{tabular}{|c|c|c|c|c|}
\hline \multirow[b]{2}{*}{ Code } & \multicolumn{2}{|c|}{ Default } & \multirow[b]{2}{*}{ Properties $^{1}$} & \multirow[b]{2}{*}{ Projection name } \\
\hline & $\phi_{0}$ & $\theta_{0}$ & & \\
\hline \multicolumn{5}{|c|}{ Zenithal (azimuthal) projections } \\
\hline AZP & $0^{\circ}$ & $90^{\circ}$ & Sect. 5.1.1 & Zenithal perspective \\
\hline SZP & $0^{\circ}$ & $90^{\circ}$ & Sect. 5.1.2 & Slant zenithal perspective \\
\hline TAN & $0^{\circ}$ & $90^{\circ}$ & Sect. 5.1 .3 & Gnomonic \\
\hline STG & $0^{\circ}$ & $90^{\circ}$ & Sect. 5.1.4 & Stereographic \\
\hline SIN & $0^{\circ}$ & $90^{\circ}$ & Sect. 5.1 .5 & Slant orthographic \\
\hline ARC & $0^{\circ}$ & $90^{\circ}$ & Sect. 5.1.6 & Zenithal equidistant \\
\hline ZPN & $0^{\circ}$ & $90^{\circ}$ & Sect. 5.1.7 & Zenithal polynomial \\
\hline ZEA & $0^{\circ}$ & $90^{\circ}$ & Sect. 5.1.8 & Zenithal equal-area \\
\hline AIR & $0^{\circ}$ & $90^{\circ}$ & Sect. 5.1 .9 & Airy \\
\hline \multicolumn{5}{|c|}{ Cylindrical projections } \\
\hline CYP & $0^{\circ}$ & $0^{\circ}$ & Sect. 5.2.1. & Cylindrical perspective \\
\hline CEA & $0^{\circ}$ & $0^{\circ}$ & Sect. 5.2.2 & Cylindrical equal area \\
\hline CAR & $0^{\circ}$ & $0^{\circ}$ & Sect. 5.2.3 & Plate carrée \\
\hline MER & $0^{\circ}$ & $0^{\circ}$ & Sect. 5.2.4 & Mercator \\
\hline \multicolumn{5}{|c|}{ Pseudo-cylindrical and related projections } \\
\hline SFL & $0^{\circ}$ & $0^{\circ}$ & Sect. 5.3.1 & Samson-Flamsteed \\
\hline PAR & $0^{\circ}$ & $0^{\circ}$ & Sect. 5.3.2 & Parabolic \\
\hline MOL & $0^{\circ}$ & $0^{\circ}$ & Sect. 5.3.3 & Mollweide \\
\hline AIT & $0^{\circ}$ & $0^{\circ}$ & Sect. 5.3.4 & Hammer-Aitoff \\
\hline \multicolumn{5}{|c|}{ Conic projections } \\
\hline COP & $0^{\circ}$ & $\theta_{a}$ & Sect. 5.4.1 & Conic perspective \\
\hline $\mathrm{COE}$ & $0^{\circ}$ & $\theta_{a}$ & Sect. 5.4.2 & Conic equal-area \\
\hline COD & $0^{\circ}$ & $\theta_{a}$ & Sect. 5.4.3 & Conic equidistant \\
\hline $\mathrm{COO}$ & $0^{\circ}$ & $\theta_{a}^{a}$ & Sect. 5.4.4 & Conic orthomorphic \\
\hline \multicolumn{5}{|c|}{ Polyconic and pseudoconic projections } \\
\hline BON & $0^{\circ}$ & $0^{\circ}$ & Sect. 5.5.1 & Bonne's equal area \\
\hline $\mathrm{PCO}$ & $0^{\circ}$ & $0^{\circ}$ & Sect. 5.5.2 & Polyconic \\
\hline \multicolumn{5}{|c|}{ Quad-cube projections } \\
\hline TSC & $0^{\circ}$ & $0^{\circ}$ & Sect. 5.6.1 & Tangential spherical cube \\
\hline $\operatorname{CSC}$ & $0^{\circ}$ & $0^{\circ}$ & Sect. 5.6.2 & COBE quadrilateralized spherical cube \\
\hline QSC & $0^{\circ}$ & $0^{\circ}$ & Sect. 5.6 .3 & Quadrilateralized spherical cube \\
\hline \multicolumn{5}{|c|}{ HEALPix grid projection } \\
\hline HPX & $0^{\circ}$ & $0^{\circ}$ & Sect. $6^{2}$ & HEALPix grid \\
\hline
\end{tabular}

Notes. ${ }^{(1)}$ Refer to the indicated section in Calabretta \& Greisen (2002) for a detailed description. ${ }^{(2)}$ This projection is defined in Calabretta \& Roukema (2007).

are less commonly used in astronomy), the additional keyword LATPOLE $a$ must be used to specify the native latitude of the celestial pole. See Calabretta \& Greisen (2002) for the transformation equations and other details.

The accepted celestial coordinate systems are: the standard equatorial (RA-- and DEC-), and others of the form $x \mathrm{LON}$ and $x$ LAT for longitude-latitude pairs, where $x$ is $\mathrm{G}$ for Galactic, E for ecliptic, $\mathrm{H}$ for helioecliptic and $\mathrm{S}$ for supergalactic coordinates. Since the representation of planetary, lunar, and solar coordinate systems could exceed the 26 possibilities afforded by the single character $x$, pairs of the form $y z \mathrm{LN}$ and $y z \mathrm{LT}$ may be used as well.

RADESYS $a$ - [character; default: FK4, FK5, or ICRS: see below]. Name of the reference frame of equatorial or ecliptic coordinates, whose value must be one of those specified in Table 24. The default value is FK4 if the value of EQUINOX $a<1984.0$, FK5 if EQUINOX $a \geq 1984.0$, or ICRS if EQUINOX $a$ is not given.

EQUINOX $a$ - [floating point; default: see below]. Epoch of the mean equator and equinox in years, whose value must be non-negative. The interpretation of epoch depends upon the value of RADESYS $a$ if present: Besselian if the value is FK4 or FK4-NO-E, Julian if the value is FK5; not applicable if the value is ICRS or GAPPT.
EPOCH - [floating point]. This keyword is deprecated and should not be used in new FITS files. It is reserved primarily to prevent its use with other meanings. The EQUINOX keyword shall be used instead. The value field of this keyword was previously defined to contain a floating-point number giving the equinox in years for the celestial coordinate system in which positions are expressed.

DATE-OBS - [floating point]. This reserved keyword is defined in Sect. 4.4.2.

MJD-OBS - [floating point; default: DATE-OBS if given, otherwise no default]. Modified Julian Date (JD - 2400000.5 ) of the observation, whose value corresponds (by default) to the start of the observation, unless another interpretation is explained in the comment field. No specific time system (e.g. UTC, TAI, etc.) is defined for this or any of the other time-related keywords. It is recommended that the TIMESYS keyword, as defined in Appendix B be used to specify the time system.

LONPOLE $a$ - [floating point; default: $\phi_{0}$ if $\delta_{0} \geq \theta_{0}, \phi_{0}+180^{\circ}$ otherwise]. Longitude in the native coordinate system of the celestial system's north pole. Normally, $\phi_{0}$ is zero unless a non-zero value has been set for PVi_1a, which is associated with the longitude axis. This default applies for all values of $\theta_{0}$, including $\theta_{0}=90^{\circ}$, although the use of non-zero values of $\theta_{0}$ are discouraged in that case. 
Table 24. Allowed values of RADESYS $a$.

\begin{tabular}{ll}
\hline \hline Value & Definition \\
\hline ICRS & International Celestial Reference System \\
FK5 & Mean place, new (IAU 1984) system \\
FK4 $^{1}$ & Mean place, old (Bessel-Newcomb) system \\
FK4-NO-E & Mean place: but without eccentricity terms \\
GAPPT & Geocentric apparent place, IAU 1984 system \\
\hline
\end{tabular}

Notes. ${ }^{(1)}$ New FITS files should avoid using these older reference systems.

LATPOLE $a$ - [floating point; default: $90^{\circ}$, or no default if $\left.\left(\theta_{0}, \delta_{0}, \phi_{\mathrm{p}}-\phi_{0}\right)=\left(0,0, \pm 90^{\circ}\right)\right]$. Latitude in the native coordinate system of the celestial system's north pole, or equivalently, the latitude in the celestial coordinate system of the native system's north pole. May be ignored or omitted in cases where LONPOLE $a$ completely specifies the rotation to the target celestial system.

\subsection{Spectral coordinate system representations}

This section discusses the conversion of intermediate world coordinates to spectral coordinates with common axes such as frequency, wavelength, and apparent radial velocity (represented here with the coordinate variables $v, \lambda$, or $v$ ). The key point for constructing spectral WCS in FITS is that one of these coordinates must be sampled linearly in the dispersion axis; the others are derived from prescribed, usually non-linear transformations. Frequency and wavelength axes may also be sampled linearly in their logarithm.

Following the convention for the CTYPEia keyword, when $i$ is the spectral axis the first four characters must specify a code for the coordinate type; for non-linear algorithms the fifth character must be a hyphen, and the next three characters must specify a predefined algorithm for computing the world coordinates from the intermediate physical coordinates. The coordinate type must be one of those specified in Table 25. When the algorithm is linear, the remainder of the CTYPEia keyword must be blank. When the algorithm is non-linear, the 3-letter algorithm code must be one of those specified in Table 26. The relationships between the basic physical quantities $v, \lambda$, and $v$, as well as the relationships between various derived quantities are given in reference Greisen et al. (2006).

The generality of the algorithm for specifying the spectral coordinate system and its representation suggests that some additional description of the coordinate may be helpful beyond what can be encoded in the first four characters of the CTYPEia keyword; CNAMEia is reserved for this purpose. Note that this keyword provides a name for an axis in a particular WCS, while the WCSNAME $a$ keyword names the particular WCS as a whole. In order to convert between some form of radial velocity and either frequency or wavelength, the keywords RESTFRQ $a$ and RESTWAV $a$, respectively, are reserved.

CNAMEia - [character; default: default: “ ” (i.e. a linear, undefined axis)]. Spectral coordinate description which must not exceed 68 characters in length.

RESTFRQ $a$ - [floating point; default: none]. Rest frequency of the of the spectral feature of interest. The physical unit must be $\mathrm{Hz}$.

RESTWAV $a$ - [floating point; default: none]. Vacuum rest wavelength of the of the spectral feature of interest. The physical unit must be m.
One or the other of RESTFRQ $a$ or RESTWAVa should be given when it is meaningful to do so.

\subsubsection{Spectral coordinate reference frames}

Frequencies, wavelengths, and apparent radial velocities are always referred to some selected standard of rest (i.e., reference frame). While the spectra are obtained they are, of necessity, in the observer's rest frame. The velocity correction from topocentric (the frame in which the measurements are usually made) to standard reference frames (which must be one of those given in Table 27) are dependent on the dot product with timevariable velocity vectors. That is, the velocity with respect to a standard reference frame depends upon direction, and the velocity (and frequency and wavelength) with respect to the local standard of rest is a function of the celestial coordinate within the image. The keywords SPECSYS $a$ and SSYSOBS $a$ are reserved and, if used, must describe the reference frame in use for the spectral axis coordinate(s) and the spectral reference frame that was held constant during the observation, respectively. In order to compute the velocities it is necessary to have the date and time of the observation; the keywords DATE-AVG and MJD-AVG are reserved for this purpose.

DATE-AVG - [character; default: none]. Calendar date of the mid-point of the observation, expressed in the same way as the DATE-OBS keyword.

MJD-AVG - [floating point; default: none]. Modified Julian Date (JD - 2400000.5) of the mid-point of the observation.

SPECSYS $a$ - [character; default: none]. The reference frame in use for the spectral axis coordinate(s). Valid values are given in Table 27.

SSYSOBS $a$ - [character; default: TOPOCENT]. The spectral reference frame that is constant over the range of the non-spectral world coordinates. Valid values are given in Table 27.

The transformation from the rest frame of the observer to a standard reference frame requires a specification of the location on Earth ${ }^{13}$ of the instrument used for the observation in order to calculate the diurnal Doppler correction due to the Earth's rotation. The location, if specified, shall be represented as a geocentric Cartesian triple with respect to a standard ellipsoidal geoid at the time of the observation. While the position can often be specified with an accuracy of a meter or better, for most purposes positional errors of several kilometers will have negligible impact on the computed velocity correction. For details, see reference Greisen et al. (2006).

OBSGEO-X $a$ - [floating point; default: none]. $X$-coordinate (in meters) of a Cartesian triplet that specifies the location, with respect to a standard, geocentric terrestrial reference frame, where the observation took place. The coordinate must be valid at the epoch MJD-AVG or DATE-AVG.

OBSGEO-Y $a$ - [floating point; default: none]. $Y$-coordinate (in meters) of a Cartesian triplet that specifies the location, with respect to a standard, geocentric terrestrial reference frame, where the observation took place. The coordinate must be valid at the epoch MJD-AVG or DATE-AVG.

OBSGEO-Z $a$ - [floating point; default: none]. $Z$-coordinate (in meters) of a Cartesian triplet that specifies the location, with respect to a standard, geocentric terrestrial reference frame,

13 The specification of location for an instrument on a spacecraft in flight requires an ephemeris; keywords that might be required in this circumstance are not defined here. 
Table 25. Reserved spectral coordinate type $\operatorname{codes}^{1}$.

\begin{tabular}{lllll}
\hline \hline Code & Type & Symbol & Assoc. variable & Default units \\
\hline FREQ & Frequency & $v$ & $v$ & $\mathrm{~Hz}$ \\
ENER & Energy & $E$ & $v$ & $\mathrm{~J}$ \\
WAVN & Wavenumber & $\kappa$ & $v$ & $\mathrm{~m}^{-1}$ \\
VRAD & Radio velocity & $V$ & $v$ & $\mathrm{~m} \mathrm{~s}^{-1}$ \\
WAVE & Vacuum wavelength & $\lambda$ & $\lambda$ & $\mathrm{m}^{-1}$ \\
VOPT & Optical velocity & $Z$ & $\lambda$ & $\mathrm{m} \mathrm{s}^{-1}$ \\
ZOPT & Redshift & $z$ & $\lambda$ & $\ldots$ \\
AWAV & Air wavelength & $\lambda_{a}$ & $\lambda_{a}$ & $\mathrm{~m} \mathrm{~s}^{-1}$ \\
VELO & Apparent radial velocity & $v$ & $v$ & $\mathrm{~m} \mathrm{~s}^{-1}$ \\
BETA & Beta factor $(v / c)$ & $\beta$ & $v$ & $\ldots$ \\
\hline
\end{tabular}

Notes. ${ }^{(1)}$ Characters 1 through 4 of the value of the keyword CTYPEia. ${ }^{(2)}$ By convention, the "radio" velocity is given by $c\left(v_{0}-v\right) / v_{0}$ and the "optical" velocity is given by $c\left(\lambda-\lambda_{0}\right) / \lambda_{0}$.

Table 26. Non-linear spectral algorithm $\operatorname{codes}^{1}$.

\begin{tabular}{lll}
\hline \hline Code & Regularly sampled in & Expressed as \\
\hline F2W & Frequency & Wavelength \\
F2V & & Apparent radial velocity \\
F2A & & Air wavelength \\
W2F & Wavelength & Frequency \\
W2V & & Apparent radial velocity \\
W2A & & Air wavelength \\
V2F & Apparent radial vel. & Frequency \\
V2W & & Wavelength \\
V2A & & Air wavelength \\
A2F & Air wavelength & Frequency \\
A2W & & Wavelength \\
A2V & & Apparent radial velocity \\
\hline LOG & Logarithm & Any four-letter type code \\
GRI & Detector & Any type code from Table 25 \\
GRA & Detector & Any type code from Table 25 \\
TAB & Not regular & Any four-letter type code \\
\hline
\end{tabular}

Notes. ${ }^{(1)}$ Characters 6 through 8 of the value of the keyword CTYPEia.

where the observation took place. The coordinate must be valid at the epoch MJD-AVG or DATE-AVG.

Information on the relative radial velocity between the observer and the selected standard of rest in the direction of the celestial reference coordinate may be provided, and if so shall be given by the VELOSYS $a$ keyword. The frame of rest defined with respect to the emitting source may be represented in FITS; for this reference frame it is necessary to define the velocity with respect to some other frame of rest. The keywords SPECSYS $a$ and ZSOURCE $a$ are used to document the choice of reference frame and the value of the systemic velocity of the source, respectively.

SSYSSRC $a$ - [character; default: none]. Reference frame for the value expressed in the ZSOURCE $a$ keyword to document the systemic velocity of the observed source. Value must be one of those given in Table 27 except for SOURCE.

VELOSYS $a$ - [floating point; default: none]. Relative radial velocity between the observer and the selected standard of rest in the direction of the celestial reference coordinate. Units must be $\mathrm{m} \mathrm{s}^{-1}$. The CUNITia keyword is not used for this purpose since the WCS version $a$ might not be expressed in velocity units.

ZSOURCE $a$ - [floating point; default: none]. Radial velocity with respect to an alternative frame of rest, expressed as a unitless redshift (i.e., velocity as a fraction of the speed of
Table 27. Spectral reference systems.

\begin{tabular}{ll}
\hline \hline Value & Definition \\
\hline TOPOCENT & Topocentric \\
GEOCENTR & Geocentric \\
BARYCENT & Barycentric \\
HELIOCEN & Heliocentric \\
LSRK & Local standard of rest (kinematic) \\
LSRD & Local standard of rest (dynamic) \\
GALACTOC & Galactocentric \\
LOCALGRP & Local Group \\
CMBDIPOL & Cosmic microwave background dipole \\
SOURCE & Source rest frame \\
\hline
\end{tabular}

Notes. These are the allowed values of the SPECSYS $a$, SSYSOBS $a$, and SSYSSRC $a$ keywords.

light in vacuum). Used in conjunction with SSYSSRC $a$ to document the systemic velocity of the observed source.

VELANGL $a$ - [floating point; default: +90.]. In the case of relativistic velocities (e.g., a beamed astrophysical jet) the transverse velocity component is important. This keyword may be used to express the orientation of the space velocity vector with respect to the plane of the sky. See Appendix A of reference Greisen et al. (2006) for further details.

\subsection{Conventional coordinate types}

The first FITS paper (Wells et al. 1981) listed a number of "suggested values" for the CTYPE $i$ keyword. Two of these have the attribute the associated world coordinates can assume only integer values and that the meaning of these integers is only defined by convention. The first "conventional" coordinate is CTYPEia = 'COMPLEX' to specify that complex values (i.e., pairs of real and imaginary components) are stored in the data array (along with an optional weight factor). Thus, the complex axis of the data array will contain two values (or three if the weight is specified). By convention, the real component has a coordinate value of 1 , the imaginary component has a coordinate value of 2 , and the weight, if any, has a coordinate value of 3 . Table 28 illustrates the required keywords for an array of 100 complex values (without weights).

The second conventional coordinate is CTYPEia = 'STOKES' to specify the polarization of the data. Conventional values, their symbols, and polarizations are given in Table 29. 
Table 28. Example keywords for a 100 element array of complex values.

\begin{tabular}{lr}
\hline \hline Keyword & \\
\hline SIMPLE $=$ & $T$ \\
BITPIX $=$ & -32 \\
NAXIS $=$ & 2 \\
NAXIS1 $=$ & 2 \\
NAXIS2 2 & 100 \\
CTYPE1 1 ' COMPLEX' & \\
CRVAL1 $1=$ & 0. \\
CRPIX1 $=$ & 0. \\
CDELT1 $=$ & 1. \\
END & \\
\hline
\end{tabular}

Table 29. Conventional stokes values.

\begin{tabular}{rcl}
\hline \hline Value & Symbol & Polarization \\
\hline 1 & $I$ & Standard Stokes unpolarized \\
2 & $Q$ & Standard Stokes linear \\
3 & $U$ & Standard Stokes linear \\
4 & $V$ & Standard Stokes circular \\
-1 & RR & Right-right circular \\
-2 & LL & Left-left circular \\
-3 & RL & Right-left cross-circular \\
-4 & LR & Left-right cross-circular \\
-5 & $X X$ & $X$ parallel linear \\
-6 & $Y Y$ & $Y$ parallel linear \\
-7 & $X Y$ & $X Y$ cross linear \\
-8 & $Y X$ & $Y X$ cross linear \\
\hline
\end{tabular}

\section{Appendix A: Syntax of keyword records}

This appendix is not part of the FITS standard but is included for convenient reference.

The following notation is used in defining the formal syntax.

$\begin{array}{ll}:= & \begin{array}{l}\text { means "is defined to be" } \\ \text { means one of } X \text { or } Y \\ \text { (no ordering relation is implied) }\end{array} \\ {[\mathrm{X}]} & \begin{array}{l}\text { means that } X \text { is } \text { optional } \\ \text { means } X \text { is repeated one or more times }\end{array} \\ \text { X... } & \begin{array}{l}\text { means the ASCII character B } \\ \text { means one of the ASCII characters A } \\ \text { "A"-"Z" }\end{array} \\ & \begin{array}{l}\text { through } Z \text { in the ASCII collating } \\ \text { sequence, as shown in Appendix D } \\ \text { means the ASCII character associated }\end{array} \\ & \begin{array}{l}\text { with the hexadecimal code nn } \\ \text { expresses a constraint or a comment } \\ \text { (it immediately follows the syntax rule) }\end{array}\end{array}$

The following statements define the formal syntax used in FITS free-format keyword records.

FITS_keyword_record :=

FITS_commentary_keyword_record |

FITS_value_keyword_record

FITS_commentary_keyword_record:= COMMENT_keyword [ascii_text_char...] |

HISTORY_keyword [ascii_text_char...] |

BLANKFIELD_keyword [ascii_text_char...]

keyword_field anychar_but_equal

[ascii_text_char...]]

keyword_field "=" anychar_but_space [ascii_text_char...]
\{Constraint: the total number of characters in a FITS_commentary_keyword_record must be exactly equal to 80.$\}$

FITS_value_keyword_record :=

keyword_field value_indicator [space...] [value] [space...] [comment]

\{Constraint: the total number of characters in a FITS_value_keyword_record must be exactly equal to 80 . $\}$

\{Comment: if the value field is not present, the value of the FITS keyword is not defined.

keyword_field :=

[keyword_char...] [space...]

\{Constraint: the total number of characters in the keyword_field must be exactly equal to 8 .

keyword_char :=

$$
\text { "A"-“Z"| “0”-“9”|“_”|“_”, }
$$

COMMENT_keyword :=

$$
\text { "C" "O" "M" "M" "E" "N" "T" space }
$$

HISTORY_keyword := "H" "I" "S" "T" "O" "R" "Y" space

BLANKFIELD_keyword := space space space space space space space space

value_indicator := "=" space

space $:=$

$$
\text { ", }
$$

comment $:=$ "/" [ascii_text_char...]

ascii_text_char := space-“ "

anychar_but_equal := space-"<"|">"_“" "

anychar_but_space := "!"_“" "

value :=

character_string_value | logical_value | integer_value | floating_value | complex_integer_value | complex_floating_value

character_string_value := begin_quote [string_text_char...] end_quote

\{Constraint: the begin_quote and end_quote are not part of the character string value but only serve as delimiters. Leading spaces are significant; trailing spaces are not.

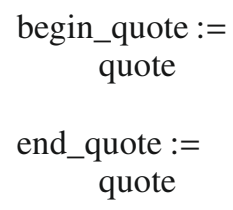


\{Constraint: the ending quote must not be immediately followed by a second quote.

$$
\begin{aligned}
& \text { quote }:= \\
& \backslash 0 \times 27
\end{aligned}
$$

string_text_char:=

$$
\text { ascii_text_char }
$$

\{Constraint: a string_text_char is identical to an ascii_text_char except for the quote char; a quote char is represented by two successive quote chars.

$$
\begin{gathered}
\text { logical_value := } \\
\text { "T"| F"F" }
\end{gathered}
$$

integer_value :=

$$
\text { [sign] digit [digit...] }
$$

\{Comment: such an integer value is interpreted as a signed decimal number. It may contain leading zeros. $\}$

$$
\begin{aligned}
& \text { sign := "_" “+" } \\
& \text { digit := } \\
& \text { "0"-“9" } \\
& \text { floating_value := } \\
& \text { decimal_number [exponent] } \\
& \text { decimal_number := } \\
& \text { [sign] [integer_part] [“." [fraction_part]] }
\end{aligned}
$$

\{Constraint: at least one of the integer_part and fraction_part must be present. \}

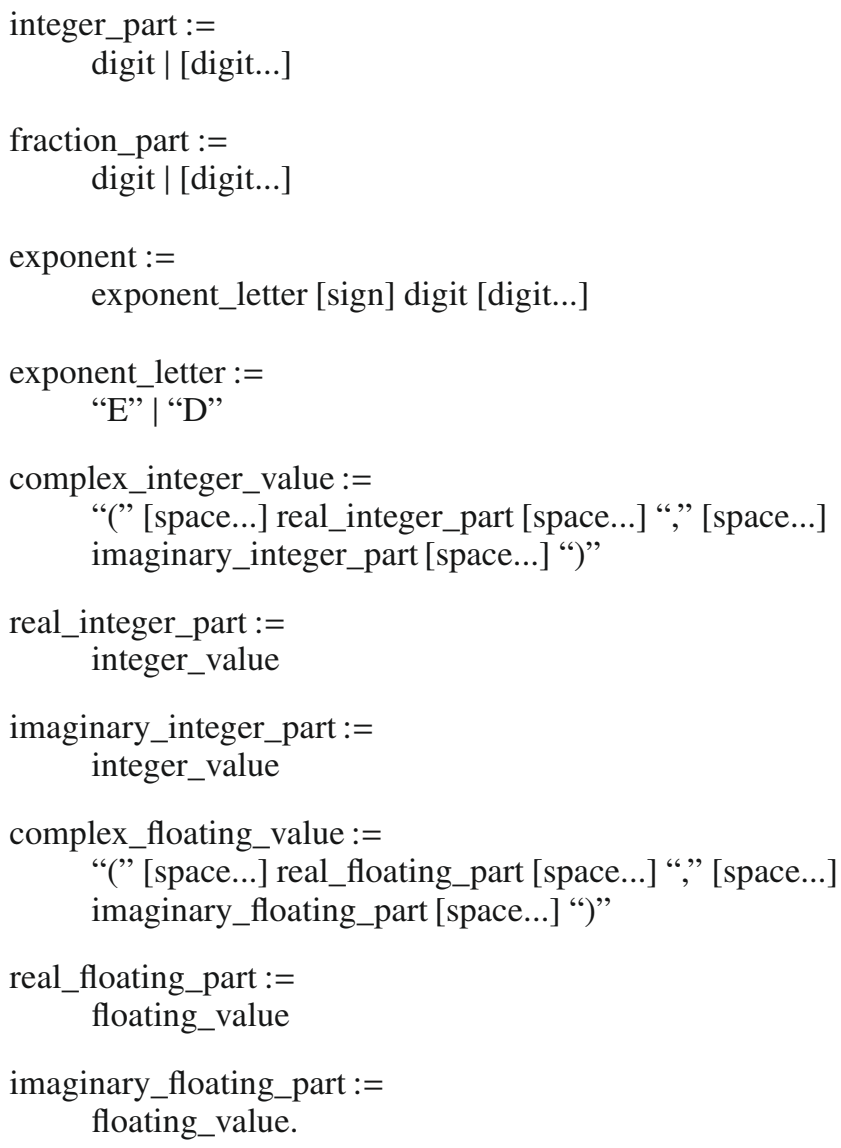

\section{Appendix B: Suggested time scale specification}

This appendix is not part of the FITS standard, but is included for convenient reference.

1. Use of the keyword TIMESYS is suggested as an implementation of the time scale specification. It sets the principal time system for time-related keywords and data in the HDU (i.e., it does not preclude the addition of keywords or data columns that provide information for transformations to other time scales, such as sidereal times or barycenter corrections). Each HDU shall contain not more than one TIMESYS keyword. Initially, officially allowed values are shown below. For reference, see: Explanatory Supplement to the Astronomical Almanac, P. K. Seidelmann, ed., University Science Books, 1992, ISBN 0-935702-68-7, or

$$
\text { http: //tycho.usno.navy.mil/systime.html }
$$

UTC - Coordinated Universal Time; defined since 1972.

UT - Universal Time, equal to Greenwich Mean Time (GMT) since 1925; the UTC equivalent before 1972; see: Explanatory Supplement, p. 76.

TAI - International Atomic Time; "UTC without the leap seconds"; 31 s ahead of UTC on 1997-07-01.

AT - International Atomic Time; deprecated synonym of TAI.

ET - Ephemeris Time, the predecessor of TT and TDB; valid until 1984.

TT - Terrestrial Time, the IAU standard time scale since 1984; continuous with ET and synchronous with (but $32.184 \mathrm{~s}$ ahead of) TAI.

TDT - Terrestrial Dynamical Time; = TT.

TDB - Barycentric Dynamical Time.

TCG - Geocentric Coordinate Time; runs ahead of TT since 1977-01-01 at a rate of approximately $22 \mathrm{~ms} /$ year.

TCB - Barycentric Coordinate Time; runs ahead of TDB since 1977-01-01 at a rate of approximately $0.5 \mathrm{~s} / \mathrm{year}$.

Use of Global Positioning Satellite (GPS) time (19 s behind TAI) is deprecated.

2. By default, times will be deemed to be as measured at the detector (or at the observatory) for time scales defined on the geoid (i.e., TAI, UTC and TT). In the case of the coordinate times TCG, TCB and TDB, the observation is assumed to have been referred to the associated spatial origin (namely the geocenter for TCG and the solar-system barycenter for TCB and TDB) by allowing for light time. These defaults follow common practice; a future convention on time scale issues in FITS files may allow other combinations but shall preserve this default behavior. The rationale is that raw observational data are most likely to be tagged by a clock that is synchronized with TAI, while a transformation to coordinate times or TDB is usually accompanied by a spatial transformation, as well. This implies that path length differences have been corrected for. Note that the same distant event recorded in a FITS file in both TDB and UTC will have times that differ by (typically) several minutes. Also, note that when the location is not unambiguous (such as in the case of an interferometer) precise specification of the location is strongly encouraged in, for instance, geocentric Cartesian coordinates.

3. Note that TT is the IAU preferred standard. It can be considered equivalent to TDT and ET, though ET should not be used for data taken after 1984. For reference, see: Explanatory Supplement, pp. 40-48. 
4. If the TIMESYS keyword is absent or has an unrecognized value, the value UTC will be assumed for dates since 1972, and UT for pre-1972 data.

5. Examples. The three legal representations of the date of October 14, 1996, might be written as:

Original format, means 1996 Oct. 14: DATE-OBS $=$ ' $14 / 10 / 96{ }^{\prime}$

Explicit time scale specification: UTC. TIMESYS $=$ 'UTC

DATE-OBS $=$ ' $1996-10-14{ }^{\prime}$

Date of start of observation, assumed in UTC DATE-OBS $=$ ' $1996-10-14$ '

Explicit time scale specification: TT.

TIMESYS $=$ 'TT

DATE-OBS $=$ ' $1996-10-14 \mathrm{~T} 10: 14: 36.123$ '

6. The convention suggested in this appendix is part of the mission-specific FITS conventions adopted for, and used in, the RXTE archive, building on existing High Energy Astrophysics FITS conventions. See the time_tutorial.html and time.html documents at http: //heasarc.gsfc.nasa.gov/docs/xte/abc/.

The VLBA project has adopted a convention where the keyword TIMSYS, rather than TIMESYS, is used, currently allowing the values UTC and IAT. See p. 38 and p. 39 of the idi-format.ps document at http://www.cv.nrao. edu/fits/documents/drafts.

\section{Appendix C: Summary of keywords}

This appendix is not part of the FITS standard, but is included for convenient reference.

All of the mandatory and reserved keywords that are defined in the standard, except for the reserved WCS keywords that are are discussed separately in Sect. 8, are listed in Tables C.1-C.3.

\section{Appendix D: ASCII text}

This appendix is not part of the FITS standard; the material in it is based on the ANSI standard for ASCII (ANSI 1977) and is included here for informational purposes.

In Table D.1, the first column is the decimal and the second column the hexadecimal value for the character in the third column. The characters hexadecimal 20 to $7 \mathrm{E}$ (decimal 32 to 126) constitute the subset referred to in this document as the restricted set of ASCII text characters.

\section{Appendix E: IEEE floating-point formats}

The material in this appendix is not part of this standard; it is adapted from the IEEE-754 floating-point standard (IEEE 1985) and provided for informational purposes. It is not intended to be a comprehensive description of the IEEE formats; readers should refer to the IEEE standard.

FITS recognizes all IEEE basic formats, including the special values.

\section{E.1. Basic formats}

Numbers in the single and double formats are composed of the following three fields:

1. 1-bit sign $s$

2. Biased exponent $e=E+$ bias

3. Fraction $f=\bullet b_{1} b_{2} \cdots b_{p-1}$.

The range of the unbiased exponent $E$ shall include every integer between two values $E_{\min }$ and $E_{\max }$, inclusive, and also two other reserved values $E_{\min }-1$ to encode \pm 0 and denormalized numbers, and $E_{\max }+1$ to encode $\pm \infty$ and NaNs. The foregoing parameters are given in Table E.1. Each nonzero numerical value has just one encoding. The fields are interpreted as follows:

\section{E.1.1. Single}

A 32-bit single format number $X$ is divided as shown in Fig. E.1. The value $v$ of $X$ is inferred from its constituent fields thus

1. If $e=255$ and $f \neq 0$, then $v$ is NaN regardless of $s$.

2. If $e=255$ and $f=0$, then $v=(-1)^{s} \infty$.

3 . If $0<e<255$, then $v=(-1)^{s} 2^{e-127}(1 \bullet f)$.

4. If $e=0$ and $f \neq 0$, then $v=(-1)^{s} 2^{e-126}(0 \bullet f)$ (denormalized numbers).

5. If $e=0$ and $f=0$, then $v=(-1)^{s} 0$ (zero).

\section{E.1.2. Double}

A 64-bit double format number $X$ is divided as shown in Fig. E.2. The value $v$ of $X$ is inferred from its constituent fields thus

1. If $e=2047$ and $f \neq 0$, then $v$ is NaN regardless of $s$.

2. If $e=2047$ and $f=0$, then $v=(-1)^{s} \infty$.

3. If $0<e<2047$, then $v=(-1)^{s} 2^{e-1023}(1 \bullet f)$.

4. If $e=0$ and $f \neq 0$, then $v=(-1)^{s} 2^{e-1022}(0 \bullet f)$ (denormalized numbers).

5. If $e=0$ and $f=0$, then $v=(-1)^{s} 0$ (zero).

\section{E.2. Byte patterns}

Table E. 2 shows the types of IEEE floating-point value, whether regular or special, corresponding to all double and single precision hexadecimal byte patterns.

\section{Appendix F: Reserved extension type names}

This appendix is not part of the FITS standard, but is included for informational purposes. It describes the extension type names registered as of the date this standard was issued. A current list is available from the FITS Support Office web site at http://fits.gsfc.nasa.gov.

\section{F.1. Standard extensions}

These three extension types have been approved by the IAUFWG and are defined in Sect. 7 of this standard document as well as in the indicated Astronomy and Astrophysics journal articles.

- 'IMAGE ing a multi-dimensional array similar to that of the FITS primary header and data unit. Approved as a standard extension in 1994 (Ponz et al. 1994). 
A\&A 524, A42 (2010)

Table C.1. Mandatory FITS keywords for the structures described in this document.

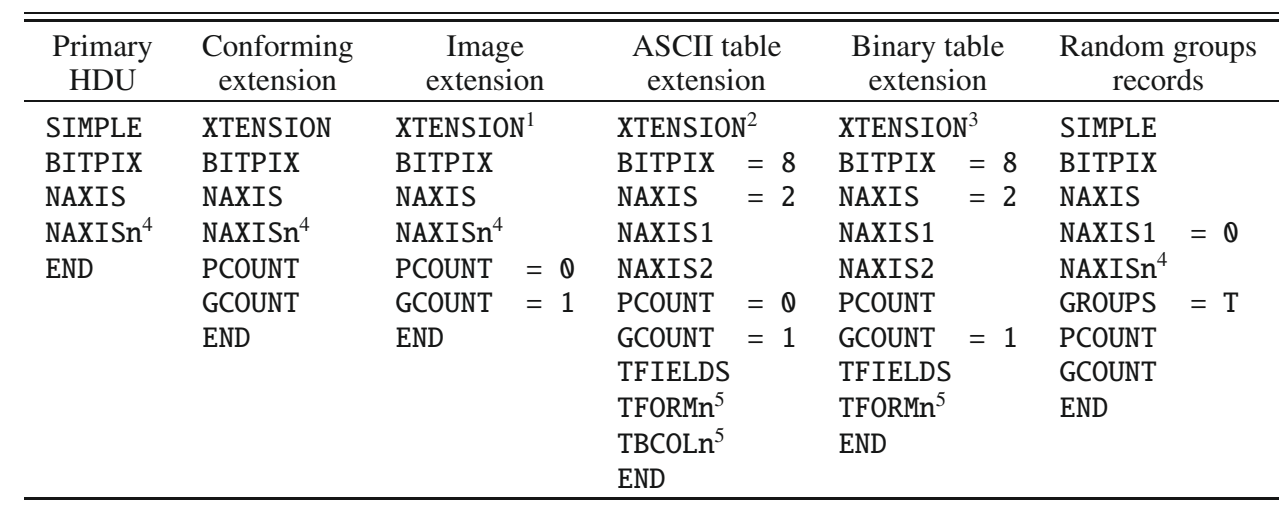

Notes. ${ }^{(1)}$ XTENSION = 'IMAGE' for the image extension. ${ }^{(2)}$ XTENSION $=$ 'TABLE' for the ASCII table extension. ${ }^{(3)}$ XTENSION $=$ 'BINTABLE' for the binary table extension. ${ }^{(4)}$ Runs from 1 through the value of NAXIS. ${ }^{(5)}$ Runs from 1 through the value of TFIELDS.

Table C.2. Reserved FITS keywords for the structures described in this document.

\begin{tabular}{|c|c|c|c|c|c|}
\hline $\begin{array}{l}\text { All }^{1} \\
\text { HDUs }\end{array}$ & $\begin{array}{l}\text { Array }^{2} \\
\text { HDUs }\end{array}$ & $\begin{array}{l}\text { Conforming } \\
\text { extension }\end{array}$ & $\begin{array}{c}\text { ASCII table } \\
\text { extension }\end{array}$ & $\begin{array}{l}\text { Binary table } \\
\text { extension }\end{array}$ & $\begin{array}{l}\text { Random groups } \\
\text { records }\end{array}$ \\
\hline$\overline{\text { DATE }}$ & BSCALE & EXTNAME & TSCALn & TSCALn & PTYPEn \\
\hline ORIGIN & BZERO & EXTVER & TZEROn & TZEROn & PSCALn \\
\hline BLOCKED $^{3}$ & BUNIT & EXTLEVEL & TNULLn & TNULLn & PZEROn \\
\hline AUTHOR & BLANK & & TTYPEn & TTYPEn & \\
\hline REFERENC & DATAMAX & & TUNITn & TUNITn & \\
\hline COMMENT & DATAMIN & & TDISPn & TDISPn & \\
\hline HISTORY & & & & TDIMn & \\
\hline பபபபபபபப) & & & & THEAP & \\
\hline DATE-OBS & & & & & \\
\hline TELESCOP & & & & & \\
\hline INSTRUME & & & & & \\
\hline OBSERVER & & & & & \\
\hline OBJECT & & & & & \\
\hline EQUINOX & & & & & \\
\hline $\mathrm{EPOCH}^{3}$ & & & & & \\
\hline EXTEND ${ }^{4}$ & & & & & \\
\hline
\end{tabular}

Notes. ${ }^{(1)}$ These keywords are further categorized in Table C.3. ${ }^{(2)}$ Primary HDU, image extension, user-defined HDUs with same array structure. (3) Deprecated. ${ }^{(4)}$ Only permitted in the primary HDU.

Table C.3. General reserved FITS keywords described in this document.

\begin{tabular}{|c|c|c|c|}
\hline Production & Bibliographic & Commentary & Observation \\
\hline DATE & AUTHOR & COMMENT & DATE-OBS \\
\hline ORIGIN & REFERENC & HISTORY & TELESCOP \\
\hline \multirow[t]{5}{*}{ BLOCKED $^{1}$} & & பபபபபபபப & INSTRUME \\
\hline & & & OBSERVER \\
\hline & & & OBJECT \\
\hline & & & EQUINOX \\
\hline & & & $\mathrm{EPOCH}^{1}$ \\
\hline
\end{tabular}

Notes. ${ }^{(1)}$ Deprecated.

\begin{tabular}{|c|c|c|c|}
\hline 1 & 8 & 23 & ....widths \\
\hline$s$ & $e$ & $f$ & \\
\hline
\end{tabular}

Fig. E.1. Single Format. msb means most significant bit, 1sb means least significant bit. 
Table D.1. ASCII character set.

\begin{tabular}{|c|c|c|c|c|c|c|c|c|c|c|c|}
\hline \multicolumn{3}{|c|}{ ASCII control } & \multicolumn{9}{|c|}{$\overline{\text { ASCII text }}$} \\
\hline dec & hex & char & dec & hex & char & dec & hex & char & dec & hex & char \\
\hline 0 & 00 & NUL & 32 & 20 & SP & 64 & 40 & a & 96 & 60 & \\
\hline 1 & 01 & $\mathrm{SOH}$ & 33 & 21 & $!$ & 65 & 41 & A & 97 & 61 & $\mathrm{a}$ \\
\hline 2 & 02 & STX & 34 & 22 & $"$ & 66 & 42 & B & 98 & 62 & $\mathrm{~b}$ \\
\hline 3 & 03 & ETX & 35 & 23 & \# & 67 & 43 & $\mathrm{C}$ & 99 & 63 & $\mathrm{c}$ \\
\hline 4 & 04 & EOT & 36 & 24 & $\$$ & 68 & 44 & D & 100 & 64 & d \\
\hline 5 & 05 & ENQ & 37 & 25 & $\%$ & 69 & 45 & E & 101 & 65 & $\mathrm{e}$ \\
\hline 6 & 06 & ACK & 38 & 26 & $\&$ & 70 & 46 & $\mathrm{~F}$ & 102 & 66 & $\mathrm{f}$ \\
\hline 7 & 07 & BEL & 39 & 27 & , & 71 & 47 & G & 103 & 67 & g \\
\hline 8 & 08 & BS & 40 & 28 & ( & 72 & 48 & $\mathrm{H}$ & 104 & 68 & $\mathrm{~h}$ \\
\hline 9 & 09 & HT & 41 & 29 & ) & 73 & 49 & I & 105 & 69 & i \\
\hline 10 & $0 \mathrm{~A}$ & LF & 42 & $2 \mathrm{~A}$ & $*$ & 74 & $4 \mathrm{~A}$ & $\mathrm{~J}$ & 106 & $6 \mathrm{~A}$ & $\mathrm{j}$ \\
\hline 11 & OB & VT & 43 & $2 B$ & + & 75 & $4 B$ & K & 107 & $6 \mathrm{~B}$ & $\mathrm{k}$ \\
\hline 12 & $0 \mathrm{C}$ & FF & 44 & $2 \mathrm{C}$ & , & 76 & $4 \mathrm{C}$ & $\mathrm{L}$ & 108 & $6 \mathrm{C}$ & 1 \\
\hline 13 & OD & CR & 45 & $2 \mathrm{D}$ & - & 77 & 4D & M & 109 & $6 \mathrm{D}$ & $\mathrm{m}$ \\
\hline 14 & $0 \mathrm{E}$ & SO & 46 & $2 \mathrm{E}$ & . & 78 & $4 \mathrm{E}$ & $\mathrm{N}$ & 110 & $6 \mathrm{E}$ & $\mathrm{n}$ \\
\hline 15 & $\mathrm{OF}$ & SI & 47 & $2 \mathrm{~F}$ & / & 79 & $4 \mathrm{~F}$ & $\mathrm{O}$ & 111 & $6 \mathrm{~F}$ & o \\
\hline 16 & 10 & DLE & 48 & 30 & 0 & 80 & 50 & $\mathrm{P}$ & 112 & 70 & $\mathrm{p}$ \\
\hline 17 & 11 & DC1 & 49 & 31 & 1 & 81 & 51 & Q & 113 & 71 & $q$ \\
\hline 18 & 12 & DC2 & 50 & 32 & 2 & 82 & 52 & $\mathrm{R}$ & 114 & 72 & $\mathrm{r}$ \\
\hline 19 & 13 & DC3 & 51 & 33 & 3 & 83 & 53 & $S$ & 115 & 73 & $\mathrm{~s}$ \\
\hline 20 & 14 & DC4 & 52 & 34 & 4 & 84 & 54 & $\mathrm{~T}$ & 116 & 74 & $\mathrm{t}$ \\
\hline 21 & 15 & NAK & 53 & 35 & 5 & 85 & 55 & $\mathrm{U}$ & 117 & 75 & $\mathrm{u}$ \\
\hline 22 & 16 & SYN & 54 & 36 & 6 & 86 & 56 & V & 118 & 76 & $\mathrm{v}$ \\
\hline 23 & 17 & ETB & 55 & 37 & 7 & 87 & 57 & W & 119 & 77 & w \\
\hline 24 & 18 & CAN & 56 & 38 & 8 & 88 & 58 & X & 120 & 78 & $\mathrm{x}$ \\
\hline 25 & 19 & EM & 57 & 39 & 9 & 89 & 59 & Y & 121 & 79 & $\mathrm{y}$ \\
\hline 26 & $1 \mathrm{~A}$ & SUB & 58 & $3 \mathrm{~A}$ & : & 90 & $5 \mathrm{~A}$ & Z & 122 & $7 \mathrm{~A}$ & $\mathrm{Z}$ \\
\hline 27 & 1B & ESC & 59 & $3 B$ & ; & 91 & $5 B$ & {[} & 123 & $7 \mathrm{~B}$ & \{ \\
\hline 28 & $1 \mathrm{C}$ & FS & 60 & $3 C$ & $<$ & 92 & $5 \mathrm{C}$ & 1 & 124 & $7 \mathrm{C}$ & | \\
\hline 29 & 1D & GS & 61 & $3 \mathrm{D}$ & $=$ & 93 & $5 \mathrm{D}$ & ] & 125 & 7D & \} \\
\hline 30 & $1 \mathrm{E}$ & RS & 62 & $3 \mathrm{E}$ & $>$ & 94 & $5 \mathrm{E}$ & $\wedge$ & 126 & $7 \mathrm{E}$ & $\sim$ \\
\hline 31 & $1 \mathrm{~F}$ & US & 63 & $3 \mathrm{~F}$ & $?$ & 95 & $5 \mathrm{~F}$ & - & 127 & $7 \mathrm{~F}$ & DEL $^{1}$ \\
\hline
\end{tabular}

Notes. ${ }^{(1)}$ Not ASCII text.

Table E.1. Summary of format parameters.

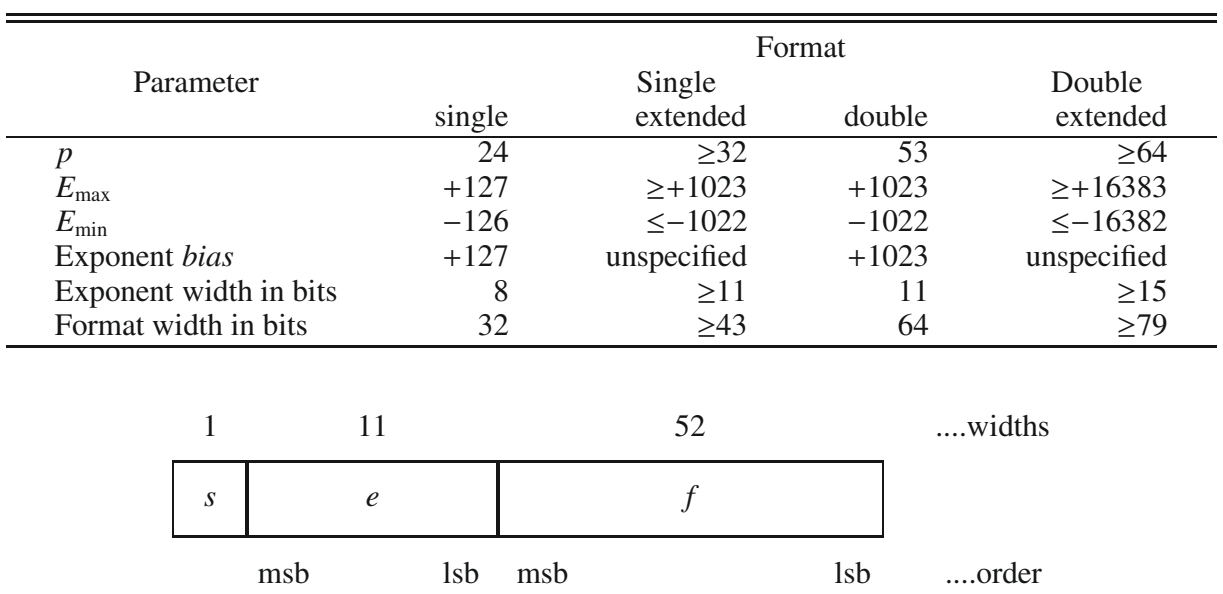

Fig. E.2. Double Format. msb means most significant bit, 1sb means least significant bit.

'TABLE ${ }_{\sqcup \sqcup \sqcup}$ ' - this ASCII table extension type contains rows and columns of data entries expressed as ASCII characters. Approved as a standard extension in 1988 (Harten et al. 1988).

'BINTABLE' - this binary table extension type provides a more flexible and efficient means of storing data structures than is provided by the TABLE extension type. The table rows can contain a mixture of numerical, logical and character data entries. In addition, each entry is allowed to be a single dimensioned array. Numeric data are kept in binary formats. Approved as a standard extension in 1994 (Cotton et al. 1995). 
Table E.2. IEEE floating-point formats.

\begin{tabular}{lcc}
\hline \hline \multicolumn{1}{c}{ IEEE value } & Double precision & Single precision \\
\hline+0 & 0000000000000000 & 00000000 \\
denormalized & 0000000000000001 & 00000001 \\
& to & to \\
& 000 FFFFFFFFFFFF & $007 \mathrm{FFFFF}$ \\
positive underflow & 0010000000000000 & 00800000 \\
positive numbers & 0010000000000001 & 00800001 \\
& to & to \\
positive overflow & 7FEFFFFFFFFFFFE & 7F7FFFFE \\
$+\infty$ & 7FEFFFFFFFFFFF & 7F7FFFFF \\
NaN ${ }^{1}$ & 7FF0000000000000 & 7F800000 \\
& 7FF0000000000001 & 7F800001 \\
to & to \\
-0 & to & 7FFFFFFF \\
negative & 8000000000000000 & 80000000 \\
denormalized & to & 80000001 \\
& 8000000000000001 & to \\
negative underflow & $800 \mathrm{FFFFFFFFFFF}$ & $807 \mathrm{FFFFF}$ \\
negative numbers & 8010000000000000 & 80800000 \\
& to & 80800001 \\
negative overflow & FFEFFFFFFFFFFF & to \\
$-\infty$ & FFEFFFFFFFFFFF & FF7FFFFF \\
NaN ${ }^{1}$ & FFF0000000000000 & FF800000 \\
& FFF0000000000001 & FF800001 \\
& to & to \\
& FFFFFFFFFFFFFF & FFFFFFF \\
\hline
\end{tabular}

Notes. ${ }^{(1)}$ Certain values may be designated as quiet $\mathrm{NaN}$ (no diagnostic when used) or signaling (produces diagnostic when used) by particular implementations.

\section{F.2. Conforming extensions}

These conventions meet the requirements for a conforming extension as defined in Sect. 3.4.1 of this standard, however they have not been formally approved or endorsed by the IAUFWG.

- 'IUEIMAGE' - this name was given to the prototype of the IMAGE extension type and was primarily used in the IUE project data archive from approximately 1992 to 1994. Except for the name, the format is identical to the IMAGE extension.

- 'A3DTABLE' - this name was given to the prototype of the BINTABLE extension type and was primarily used in the AIPS data processing system developed at NRAO from about 1987 until it was replaced by BINTABLE in the early 1990s. The format is defined in the "Going AIPS" manual (Cotton et al. 1990), Chap. 14. It is very similar to the BINTABLE type except that it does not support the variablelength array convention.

- 'FOREIGN ' $^{-}$this extension type is used to put a FITS wrapper about an arbitrary file, allowing a file or tree of files to be wrapped up in FITS and later restored to disk. A full description of this extension type is given in the Registry of FITS conventions on the FITS Support Office web site.

- 'DUMP stream of binary data values. The only known use of this extension type is to record telemetry header packets for data from the Hinode mission. The more general FOREIGN extension type could also be used to store this type of data.

\section{F.3. Other suggested extension names}

There have been occasional suggestions for other extension names that might be used for other specific purposes. These include a COMPRESS extension for storing compressed images, a FITS extension for hierarchically embedding entire FITS files within other FITS files, and a FILEMARK extension for representing the equivalent of an end-of-file mark on magnetic tape media. None of these extension types have been implemented or used in practice, therefore these names are not reserved. These extension names (or any other extension name not specifically mentioned in the previous sections of this appendix) should not be used in any FITS file without first registering the name with the IAU FITS Working Group.

\section{Appendix G: MIME types}

This appendix is not part of the FITS standard, but is included for informational purposes.

RFC 4047 (Allen \& Wells 2005) describes the registration of the Multipurpose Internet Mail Extensions (MIME) sub-types "application/fits" and "image/fits" to be used by the international astronomical community for the interchange of FITS files. The MIME type serves as a electronic tag or label that is transmitted along with the FITS file that tells the receiving application what type of file is being transmitted. The remainder of this appendix has been extracted verbatim from the RFC 4047 document.

The general nature of the full FITS standard requires the use of the media type "application/fits". Nevertheless, the principal intent for a great many FITS files is to convey a single 
data array in the primary HDU, and such arrays are very often 2-dimensional images. Several common image viewing applications already display single-HDU FITS files, and the prototypes for virtual observatory projects specify that data provided by web services be conveyed by the data array in the primary HDU. These uses justify the registration of a second media type, namely "image/fits", for files which use the subset of the standard described by the original FITS standard paper. The MIME type "image/fits" may be used to describe FITS primary HDUs that have other than two dimensions, however it is expected that most files described as "image/fits" will have two-dimensional (NAXIS $=2$ ) primary HDUs.

\section{G.1. MIME type "application/fits"}

A FITS file described with the media type "application/ fits" should conform to the published standards for FITS files as determined by convention and agreement within the international FITS community. No other constraints are placed on the content of a file described as "application/fits".

A FITS file described with the media type "application/ fits" may have an arbitrary number of conforming extension HDUs that follow its mandatory primary header and data unit. The extension HDUs may be one of the standard types (IMAGE, TABLE, and BINTABLE) or any other type that satisfies the "Requirements for Conforming Extensions" (Sect. 3.4.1). The primary HDU or any IMAGE extension may contain zero to 999 dimensions with zero or more pixels along each dimension.

The primary HDU may use the random groups convention, in which the dimension of the first axis is zero and the keywords GROUPS, PCOUNT and GCOUNT appear in the header. NAXIS1 $=0$ and GROUPS $=\mathrm{T}$ is the signature of random groups; see Sect. 6.

\section{G.1.1. Recommendations for application writers}

An application intended to handle "application/fits" should be able to provide a user with a manifest of all of the HDUs that are present in the file and with all of the keyword/value pairs from each of the HDUs.

An application intended to handle "application/fits" should be prepared to encounter extension HDUs that contain either ASCII or binary tables, and to provide a user with access to their elements.

An application which can modify FITS files or retrieve FITS files from an external service should be capable of writing such files to a local storage medium.

Complete interpretation of the meaning and intended use of the data in each of the HDUs typically requires the use of heuristics that attempt to ascertain which local conventions were used by the author of the FITS file.

As examples, files with media type "application/fits" might contain any of the following contents:

- An empty primary HDU (containing zero data elements) followed by a table HDU that contains a catalog of celestial objects.

- An empty primary HDU followed by a table HDU that encodes a series of time-tagged photon events from an exposure using an X-ray detector.

- An empty primary HDU followed by a series of IMAGE HDUs containing data from an exposure taken by a mosaic of CCD detectors.
- An empty primary HDU followed by a series of table HDUs that contain a snapshot of the state of a relational database.

- A primary HDU containing a single image along with keyword/value pairs of metadata.

- A primary HDU with NAXIS1 $=0$ and GROUPS $=\mathrm{T}$ followed by random groups data records of complex fringe visibilities.

\section{G.2. MIME type "image/fits"}

A FITS file described with the media type "image/fits" should have a primary HDU with positive integer values for the NAXIS and NAXISn keywords, and hence should contain at least one pixel. Files with 4 or more non-degenerate axes (NAXISn > 1) should be described as "application/fits", not as "image/fits". (In rare cases it may be appropriate to describe a NULL image - a dataless container for FITS keywords, with NAXIS $=\theta$ or NAXISn $=\theta-$ or an image with $4+$ non-degenerate axes as "image/fits" but this usage is discouraged because such files may confuse simple image viewer applications.)

FITS files declared as "image/fits" may also have one or more conforming extension HDUs following their primary HDUs. These extension HDUs may contain standard, non-linear, world coordinate system (WCS) information in the form of tables or images. The extension HDUs may also contain other, non-standard metadata pertaining to the image in the primary HDU in the forms of keywords and tables.

A FITS file described with the media type "image/fits" should be principally intended to communicate the single data array in the primary HDU. This means that "image/fits" should not be applied to FITS files containing multi-exposureframe mosaic images. Also, random groups files must be described as "application/fits" and not as "image/fits".

A FITS file described with the media type "image/fits" is also valid as a file of media type "application/fits". The choice of classification depends on the context and intended usage.

\section{G.2.1. Recommendations for application writers}

An application that is intended to handle "image/fits" should be able to provide a user with a manifest of all of the HDUs that are present in the file and with all of the keyword/value pairs from each of the HDUs. An application writer may choose to ignore HDUs beyond the primary HDU, but even in this case the application should be able to present the user with the keyword/value pairs from the primary HDU.

Note that an application intended to render "image/fits" for viewing by a user has significantly more responsibility than an application intended to handle, e.g., "image/tiff" or "image/gif". FITS data arrays contain elements which typically represent the values of a physical quantity at some coordinate location. Consequently they need not contain any pixel rendering information in the form of transfer functions, and there is no mechanism for color look-up tables. An application should provide this functionality, either statically using a more or less sophisticated algorithm, or interactively allowing a user various degrees of choice.

Furthermore, the elements in a FITS data array may be integers or floating-point numbers. The dynamic range of the data array values may exceed that of the display medium and the eye, and their distribution may be highly nonuniform. Logarithmic, 
square-root, and quadratic transfer functions along with histogram equalization techniques have proved helpful for rendering FITS data arrays. Some elements of the array may have values which indicate that their data are undefined or invalid; these should be rendered distinctly. Via WCS Paper I (Greisen $\&$ Calabretta 2002) the standard permits CTYPEn = 'COMPLEX' to assert that a data array contains complex numbers (future revisions might admit other elements such as quaternions or general tensors).

Three-dimensional data arrays (NAXIS $=3$ with NAXIS1, NAXIS2 and NAXIS3 all greater than 1) are of special interest. Applications intended to handle "image/fits" may default to displaying the first 2D plane of such an image cube, or they may default to presenting such an image in a fashion akin to that used for an animated GIF, or they may present the data cube as a mosaic of "thumbnail" images. The time-lapse movie-looping display technique can be effective in many instances, and application writers should consider offering it for all three-dimensional arrays.

An "image/fits" primary HDU with NAXIS = 1 is describing a one-dimensional entity such as a spectrum or a time series. Applications intended to handle "image/fits" may default to displaying such an image as a graphical plot rather than as a two-dimensional picture with a single row.

An application that cannot handle an image with dimensionality other than two should gracefully indicate its limitations to its users when it encounters NAXIS $=1$ or NAXIS $=3$ cases, while still providing access to the keyword/value pairs.

FITS files with degenerate axes (i.e., one or more NAXISn = 1) may be described as "image/fits", but the first axes should be non-degenerate (i.e., the degenerate axes should be the highest dimensions). An algorithm designed to render only two-dimensional images will be capable of displaying such an NAXIS $=3$ or NAXIS $=4$ FITS array that has one or two of the axes consisting of a single pixel, and an application writer should consider coding this capability into the application. Writers of new applications that generate FITS files intended to be described as "image/fits" should consider using the WCSAXES keyword (Greisen et al. 2006) to declare the dimensionality of such degenerate axes, so that NAXIS can be used to convey the number of non-degenerate axes.

\section{G.3. File extensions}

The FITS standard originated in the era when files were stored and exchanged via magnetic tape; it does not prescribe any nomenclature for files on disk. Various sites within the FITS community have long-established practices where files are presumed to be FITS by context. File extensions used at such sites commonly indicate content of the file instead of the data format.

In the absence of other information it is reasonably safe to presume that a file name ending in ".fits" is intended to be a FITS file. Nevertheless, there are other commonly used extensions; e.g., ".fit", ".fts", and many others not suitable for listing in a media type registration.

\section{Appendix H: Past changes or clarifications to the formal definition of FITS}

This appendix is not part of the FITS standard, but is included for informational purposes. This appendix describes known differences between the requirements in this standard and the requirements in the original FITS papers:

- Section 4.1.2: the original FITS definition paper (Wells et al. 1981) disallows lower case letters in the keyword name, but does not specify what other characters may or may not appear in the name.

- Section 4.1.2: the slash between the value and comment is "recommended" in the original paper (Wells et al. 1981) whereas the standard requires that it be present, which is consistent with the prescription of Fortran list-directed input.

- Section 4.2: the original paper (Wells et al. 1981) speculated that FITS would eventually support the full range of flexibility that is allowed by Fortran list-directed input, including dimensioned parameters. The standard restricts the value field to a single value, not an array.

- Sections 4.2.5 and 4.2.6: the original paper (Wells et al. 1981) defined a fixed format for complex keyword values, with the real part right justified in bytes 11 through 30 and the imaginary part right justified in bytes 31 through 50 . There are no known FITS files that use this fixed format. The standard does not define a fixed format for complex keyword values. Instead, complex values are represented in conformance with the rules for Fortran list-directed input, namely, with the real and imaginary parts separated by a comma and enclosed in parentheses.

- Sections 4.4.1.1 and 4.4.1.2: the paper that defines generalized extensions (Grosbøl et al. 1988) does not prohibit the appearance of the SIMPLE keyword in extensions nor the XTENSION keyword in the primary header.

\section{References}

Allen, S., \& Wells, D. 2005, IETF RFC 4047, http://www.ietf.org/rfc/ rfc4047.txt

ANSI 1977, American National Standard for Information Processing: Code for Information Interchange, ANSI X3.4-1977 (ISO 646), New York: American National Standards Institute, Inc.

Bradner, S. 1997, IETF RFC 2119, http://www.ietf.org/rfc/rfc2119. txt

Bunclark, P., \& Rots, A. 1997, Precise re-definition of DATE-OBS Keyword encompassing the millennium, http://fits.gsfc.nasa.gov/year2000. html

Calabretta, M. R., \& Greisen, E. W. 2002, A\&A, 395, 1077

Calabretta, M. R., \& Roukema, B. F. 2007, MNRAS, 381, 865

Cotton, W. D., et al. 1990, Going AIPS: A Programmer's Guide to the NRAO Astronomical Image Processing System, Charlottesville: NRAO

Cotton, W. D., Tody, D. B., \& Pence, W. D. 1995, A\&AS, 113, 159

Greisen, E. W., \& Harten, R. H. 1981, A\&AS, 44, 371

Greisen, E. W., \& Calabretta, M. R. 2002, A\&A, 395, 1061

Greisen, E. W., Calabretta, M. R., Valdes, F. G., \& Allen, S. L. 2006, A\&A, 446, 747

Grosbøl, P., \& Wells, D. C. 1994, Blocking of Fixed-block Sequential Media and Bitstream Devices, http://fits.gsfc.nasa.gov/blocking94.html

Grosbøl, P., Harten, R. H., Greisen, E. W., \& Wells, D. C. 1988, A\&AS, 73, 359 Hanisch, R. J., Farris, A., Greisen, E. W., et al. 2001, A\&A, 376, 359

Harten, R. H., Grosbøl, P., Greisen, E. W., \& Wells, D. C. 1988, A\&AS, 73, 365 IAU 1983, Transactions of the IAU, XVIIIB, 45

IAU 1988, Transactions of the IAU, XXB, 51

IEEE 1985, American National Standard - IEEE Standard for Binary Floating Point Arithmetic, ANSI/IEEE 754-1985, New York: American National Standards Institute, Inc.

ISO 2004, Information technology - Programming languages - Fortran, ISO/IEC 1539-1:2004, Geneva: International Organization for Standardization

McNally, D. 1988, Transactions of the IAU, Proceedings of the Twentieth General Assembly (Dordrecht: Kluwer)

Ponz, J. D., Thompson, R. W., \& Muñoz, J. R. 1994, A\&AS, 105, 53 Schmitz, M., Helou, G., Dubois, P., et al. 1995, Information \& On-line data in Astronomy, ed. D. Egret, \& M. A. Albrecht (Kluwer Academic Pub.), 259

Wells, D. C., Greisen, E. W., \& Harten, R. H. 1981, A\&AS, 44, 363

Wells, D. C., \& Grosbøl, P. 1990, Floating Point Agreement for FITS, http : // fits.gsfc.nasa.gov/fp89.txt 